\title{
Rotation of Late-type Stars in Praesepe with K2
}

\author{
L. M. Rebull ${ }^{1,2}$, J. R. Stauffer ${ }^{2}$, L. A. Hillenbrand ${ }^{3}$, A. M. Cody ${ }^{4}$, J. Bouvier ${ }^{5,6}$, \\ D. R. Soderblom ${ }^{7,8}$, M. Pinsonneault ${ }^{9,10}$, and L. Hebb ${ }^{11}$ \\ ${ }^{1}$ Infrared Science Archive (IRSA), Infrared Processing and Analysis Center (IPAC), 1200 East California Boulevard, \\ California Institute of Technology, Pasadena, CA 91125, USA; rebull@ipac.caltech.edu \\ ${ }^{2}$ Spitzer Science Center (SSC), Infrared Processing and Analysis Center (IPAC), 1200 East California Boulevard, \\ California Institute of Technology, Pasadena, CA 9112, USA \\ ${ }^{3}$ Astronomy Department, California Institute of Technology, Pasadena, CA 91125, USA \\ ${ }^{4}$ NASA Ames Research Center, Kepler Science Office, Mountain View, CA 94035, USA \\ ${ }^{5}$ Université de Grenoble, Institut de Planétologie et d'Astrophysique de Grenoble (IPAG), F-38000 Grenoble, France \\ ${ }^{6}$ CNRS, IPAG, F-38000 Grenoble, France \\ ${ }^{7}$ Space Telescope Science Institute, 3700 San Martin Drive, Baltimore, MD 21218, USA \\ ${ }^{8}$ Center for Astrophysical Sciences, Johns Hopkins University, 3400 North Charles Street, Baltimore, MD 21218, USA \\ Department of Astronomy, The Ohio State University, Columbus, OH 43210, USA \\ ${ }^{10}$ Center for Cosmology and Astroparticle Physics, The Ohio State University, Columbus, OH 43210, USA \\ ${ }^{11}$ Department of Physics, Hobart and William Smith Colleges, Geneva, NY 14456, USA \\ Received 2017 January 30; revised 2017 March 14; accepted 2017 March 20; published 2017 April 20
}

\begin{abstract}
We have Fourier-analyzed 941 K2 light curves (LCs) of likely members of Praesepe, measuring periods for $86 \%$ and increasing the number of rotation periods $(P)$ by nearly a factor of four. The distribution of $P$ versus $\left(V-K_{\mathrm{s}}\right)$, a mass proxy, has three different regimes: $\left(V-K_{\mathrm{s}}\right)<1.3$, where the rotation rate rapidly slows as mass decreases; $1.3<\left(V-K_{\mathrm{s}}\right)<4.5$, where the rotation rate slows more gradually as mass decreases; and $\left(V-K_{\mathrm{s}}\right)>4.5$, where the rotation rate rapidly increases as mass decreases. In this last regime, there is a bimodal distribution of periods, with few between $\sim 2$ and $\sim 10$ days. We interpret this to mean that once $M$ stars start to slow down, they do so rapidly. The K2 period-color distribution in Praesepe ( $790 \mathrm{Myr})$ is much different than that in the Pleiades ( 125 Myr) for late F, G, K, and early-M stars; the overall distribution moves to longer periods and is better described by two line segments. For mid-M stars, the relationship has a similarly broad scatter and is steeper in Praesepe. The diversity of LCs and of periodogram types is similar in the two clusters; about a quarter of the periodic stars in both clusters have multiple significant periods. Multi-periodic stars dominate among the higher masses, starting at a bluer color in Praesepe $\left(\left(V-K_{\mathrm{s}}\right) \sim 1.5\right)$ than in the Pleiades $\left(\left(V-K_{\mathrm{s}}\right) \sim 2.6\right)$. In Praesepe, there are relatively more LCs that have two widely separated periods, $\Delta P>6$ days. Some of these could be examples of $\mathrm{M}$ star binaries where one star has spun down but the other has not.
\end{abstract}

Key words: stars: rotation - stars: variables: general

Supporting material: machine-readable table

\section{Introduction}

Praesepe (M44 = NGC $2632=$ the Beehive cluster) and the Hyades are often considered as twin open clusters because they appear to have essentially the same age $(\sim 790 \mathrm{Myr})$ and metallicity $([\mathrm{Fe} / \mathrm{H}] \sim 0.15)$. Some authors attribute these similarities to the two clusters having been born within the same molecular cloud or cloud complex (Schwarzschild \& Hertzsprung 1913; Klein-Wassink 1927); the space motions appear to support this picture (see, e.g., Eggen 1992). Because the Hyades is much closer than Praesepe ( 44 pc versus $~ 180$ pc; Perryman et al. 1998, van Leeuwen 2007), its stars are much brighter; the Hyades has therefore received much more attention in the published literature. However, proximity also has its disadvantages. The Hyades is spread out over a very large area on the sky, making it difficult to take advantage of multi-object spectrographs or wide-field cameras to efficiently survey many cluster members at once. Praesepe's four times greater distance can become an advantage in that sense, if a suitable wide-area facility becomes available. We have taken advantage of one such circumstance, the unexpected availability of the 100 square degree FOV Kepler Space Telescope to obtain synoptic photometry of ecliptic plane fields for $\sim 75$ days.
There have been four prior large surveys to determine rotation periods for low-mass stars in Praesepe. Scholz et al. (2011) reported 50 periods; Delorme et al. (2011) used data from the WASP telescope network to monitor $\sim 70$ members, Kovács et al. (2014) monitored 400 members with the HAT telescope network, and a pair of papers by Agüeros et al. (2011) and Douglas et al. (2014) used data from the PTF telescope to monitor $\sim 500$ members. Those groups reported periods for 49, 52, 180, and 40 Praesepe members, respectively (and a total of 220 unique stars with periods), primarily covering stars with spectral types $\mathrm{G}, \mathrm{K}$, and early $\mathrm{M}$.

The K2 (Howell et al. 2014) data for Praesepe were obtained in 2015 in Campaign 5. K2 provides precision, sensitivity, and long-duration, continuous coverage that yields superb light curves (LCs) of much greater quality than can be obtained from the ground, and extending to much lower signal amplitudes and masses. At least three groups have already obtained rotation periods for Praesepe members based on these data. Libralato et al. (2016) derived rotation periods as a step in searching the LCs for exoplanet transits, but they did not discuss the periods nor the shapes of the phased LCs. Mann et al. (2016a) similarly derive rotation periods while searching for exoplanet transits; they illustrate the distribution of rotation periods as a function 


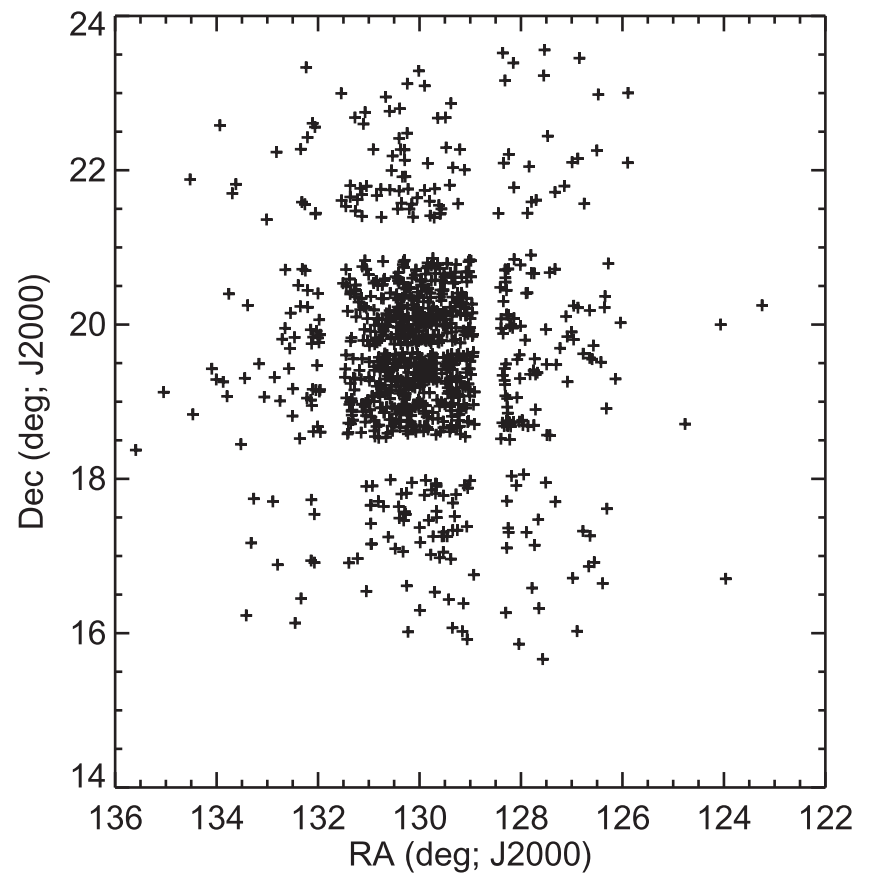

Figure 1. All 984 members or candidate members (see Section 2.5.4) of Praesepe with K2 LCs projected onto the sky. Note the gaps between K2 detectors. Stars that are $>5^{\circ}$ from the cluster center are highlighted in Appendix $\mathrm{H}$.

of $M_{k}$ and color, but do not otherwise discuss the periods or LC morphology. Finally, our group plotted an early version of the Praesepe period distribution in Stauffer et al. (2016) in the context of comparing the Pleiades rotation periods to those in other clusters. However, no paper has yet published a complete analysis of the K2 Praesepe rotational data nor a discussion of the LC morphologies shown by those stars. This paper is devoted to these two topics.

Much of our current analysis is very similar to that we conducted in the Pleiades (Rebull et al. 2016a, 2016b; Stauffer et al. 2016), and we often make comparisons to the Pleiades analysis and results. In Section 2, we present the observations and data reduction, as well as the range of data from the literature that we assembled, and define the final sample of members that we analyze in the rest of the paper. The overall distribution of K2-derived rotation rates is discussed in Section 3. Section 4 places these LCs in the same categories as we defined in the Pleiades (Rebull et al. 2016a). Section 5 compares the locations of the single- and multi-period stars in a variety of ways, and Section 6 looks at the spacings between periods for those stars with clear multiple periods. Finally, we summarize our results in Section 7.

\section{Observations and Methods}

\subsection{K2 Data}

Praesepe members and candidate members were observed in K2 Campaign 5, which lasted for 75 days between 2015 April and October. Figure 1 shows the distribution of objects with K2 LCs on the sky; note the gaps between detectors. All of the stars shown were observed in the long-cadence ( $\sim 30$ minute exposure) mode. Some of these stars were additionally observed in fast cadence ( $\sim 1$ minute exposure), but those data are beyond the scope of the present paper. There are 984 unique K2 long-cadence LCs corresponding to members or candidate members of Praesepe (see Section 2.5.4 below). The tidal radius of Praesepe is 12.1 pc (Holland et al. 2000), which at a distance of $184 \mathrm{pc}$ (see Section 2.5 below) is $\sim 3^{\circ} .8$ across, which is easily covered by K2. (Stars that are more than $5^{\circ}$ away from the cluster center are highlighted in Appendix H.)

Kepler pixel sizes are relatively large, 3 ." $98 \times 3$." 98 , and the $95 \%$ encircled energy diameter ranges from 3.1 to 7.5 pixels with a median value of 4.2 pixels. During the K2 portion of the mission, because only two reaction wheels can be used, the whole spacecraft slowly drifts and then repositions regularly every 0.245 days. This drift is $\sim 0$ " 1 per hour (Cody et al. 2017).

Since these data were reduced at the same time as we reduced our Pleiades data (Rebull et al. 2016b), we have the same sets of LCs available to us: (1) the pre-search data conditioning (PDC) version generated by the Kepler project and obtained from MAST, the Mikulski Archive for Space Telescopes, (2) a version with moving apertures obtained following A. M. Cody et al. (2017, in preparation), (3) the version using a semiparametric Gaussian process model used by Aigrain et al. $(2015,2016)$, and (4) the "self-flat-fielding" approach used by Vanderburg \& Johnson (2014) as obtained from MAST. To this, late in the process, we added (5) the LCs from the EVEREST pipeline (Luger et al. 2016), which uses pixel level decorrelation. We removed any data points corresponding to thruster firings and any others with bad data flags set in the corresponding data product.

Again, following the same approach as in the Pleiades, we inspected LCs from each reduction approach, and we selected the visually "best" LC from among the original four LC versions. Since the EVEREST LCs became available late in our analysis process, for the most part we used the EVEREST LCs to break ties or clarify what the LC was doing. About $7 \%$ had such severe artifacts that no "best" LC could be identified; as for the Pleiades, this is often a result of instrumental (nonastrophysical) artifacts (because the star is too bright or too faint or adversely affected by nearby stars, etc.). As for the Pleiades, though, the periods we report here are not generally ambiguous, and are detected in all the LC versions; they just look cosmetically better in one version or another. Our approach of comparing several different LC versions also minimizes the likelihood that any of these LC reductions removes stellar signal.

Out of the 984 LCs, only one pair of stars for which a K2 LC was requested were within $4^{\prime \prime}$ of each other (within a Kepler pixel). These objects are discussed further in Appendix A.

\subsection{Finding Periods}

Our approach for finding periods was identical to what we used in the Pleiades in Rebull et al. (2016b). In summary, we used the Lomb-Scargle (LS; Scargle 1982) approach as implemented by the NASA Exoplanet Archive Periodogram Service $^{12}$ (Akeson et al. 2013). We looked for periods between 0.05 and 35 days, with the upper limit being set by roughly half the campaign length.

Figure 2 shows LCs, periodograms, and phased LCs for some Praesepe stars with single, unambiguous periods. As we found in the Pleiades, the overwhelming majority of them have

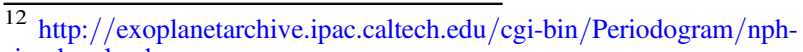
simpleupload
} 

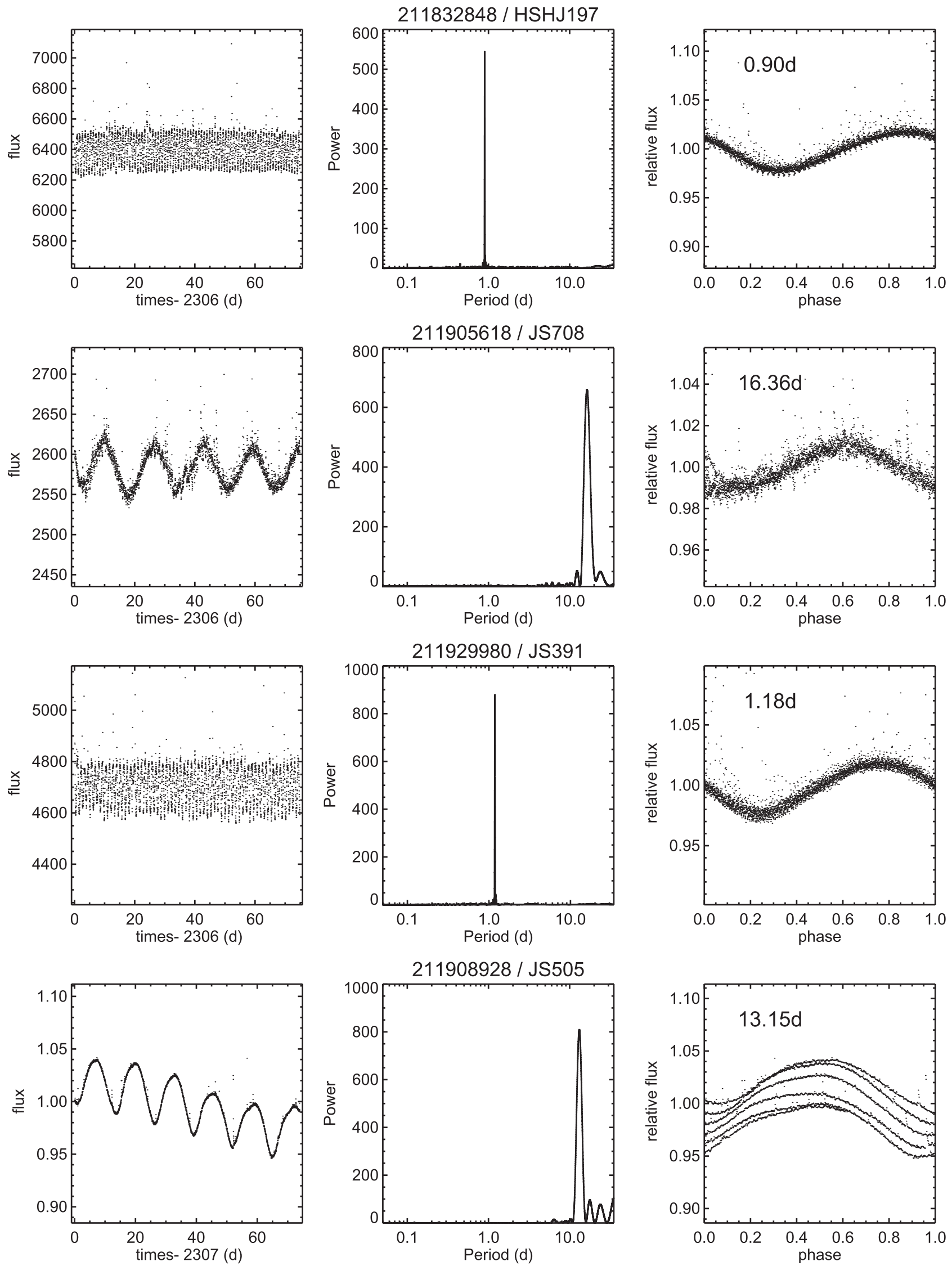

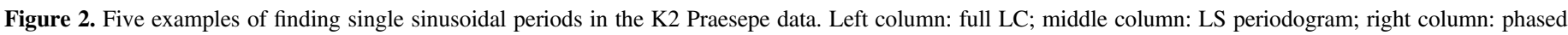

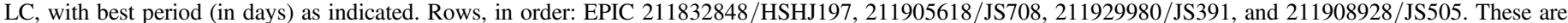

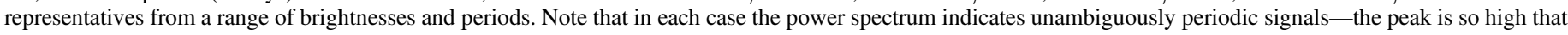

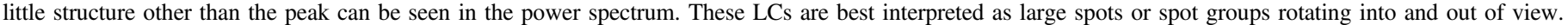


zero, or effectively zero, false alarm probability (FAP); a significant fraction have more than one peak with $0 \mathrm{FAP}-$ see Section 4 below.

\subsection{Interpretation of Periods}

For stars of the mass range considered here, the phased LCs are mostly sinsuoidal and therefore best attributed to star spotmodulated rotation. We find periods for 828/984 K2 LCs, or $84 \%$ of all K2 LCs of candidate or confirmed Praesepe members (see Section 2.5.4). There is no significant trend with color (as a proxy for mass) for the fraction of periodic stars.

As for the Pleiades, we removed from this distribution any periods that are likely to be eclipsing binaries (EBs; see Appendix B) or those whose waveforms did not seem to be rotation periods (see Appendix C).

There are a few multi-periodic stars that we suspect to be pulsating variables (see Appendix D); many of them are also reported in the literature as $\delta$ Scuti-type variables. For some pulsators, the first period is still likely to be similar to the rotation period (see discussion in Rebull et al. 2016a). For the remaining multi-periodic stars $(\sim 25 \%$ of the sample; see Section 4 below), for the most part, we took the period corresponding to the strongest peak in the periodogram as the rotation period to be used for subsequent analysis. In a few cases (e.g., double-dip stars; see Section 5), a secondary peak is the right $P_{\text {rot }}$ to use.

\subsection{Comparison to Literature Periods}

We have chosen four of the most recent surveys looking for rotation rates in Praesepe for a detailed comparison of our results; they are summarized in Figure 3. There are 220 Praesepe stars in the literature with at least one estimate of $P_{\text {rot }}$; 60 of those do not have K2 LCs.

Scholz et al. (2011) used the Isaac Newton Telescope to monitor very low mass members, reporting 49 periods. We have periods for 40 of the stars in common between the two studies; the remaining Scholz et al. stars were not monitored (either because they are too faint for $\mathrm{K} 2$ or because they are in the gaps between chips). The periods derived for these 40 stars are shown in Figure 3 . There is generally very good agreement; the median fractional difference $\left(\left|P_{\text {Scholz }+}-P_{\text {here }}\right| / P_{\text {here }}\right)$ is $2.4 \%$. There are eight stars for which their period and ours do not match, and most of them are harmonics, because they are off by factors of 2 (too small or large). In four of these cases, the period we derive is very close to 1 day, which is hard to recover with a ground-based telescope. We believe our periods to be correct in all cases for the epochs in which we observed this cluster. Two stars are of special note. EPIC $211951438 / \mathrm{HSHJ} 396$ has a period of 0.593 days in Scholz et al., but we cannot recover any period for this star, so we do not retain it as periodic. EPIC 211984058/2MASSJ08384128 +1959471 was not a star for which we initially found a period, because all five LC versions are different, and it was not clear which LC version is the "best" (see discussion above in Section 2). However, upon examination of the K2 thumbnail and POSS images of the region, several of the LC versions appear to have been dragged off to a nearby bright star. The Aigrain et al. version is the only one that both stays on the target star and has a periodic signal. The period we derive from this is identical to that from Scholz et al. (2011), so we retained this star as periodic with that period.

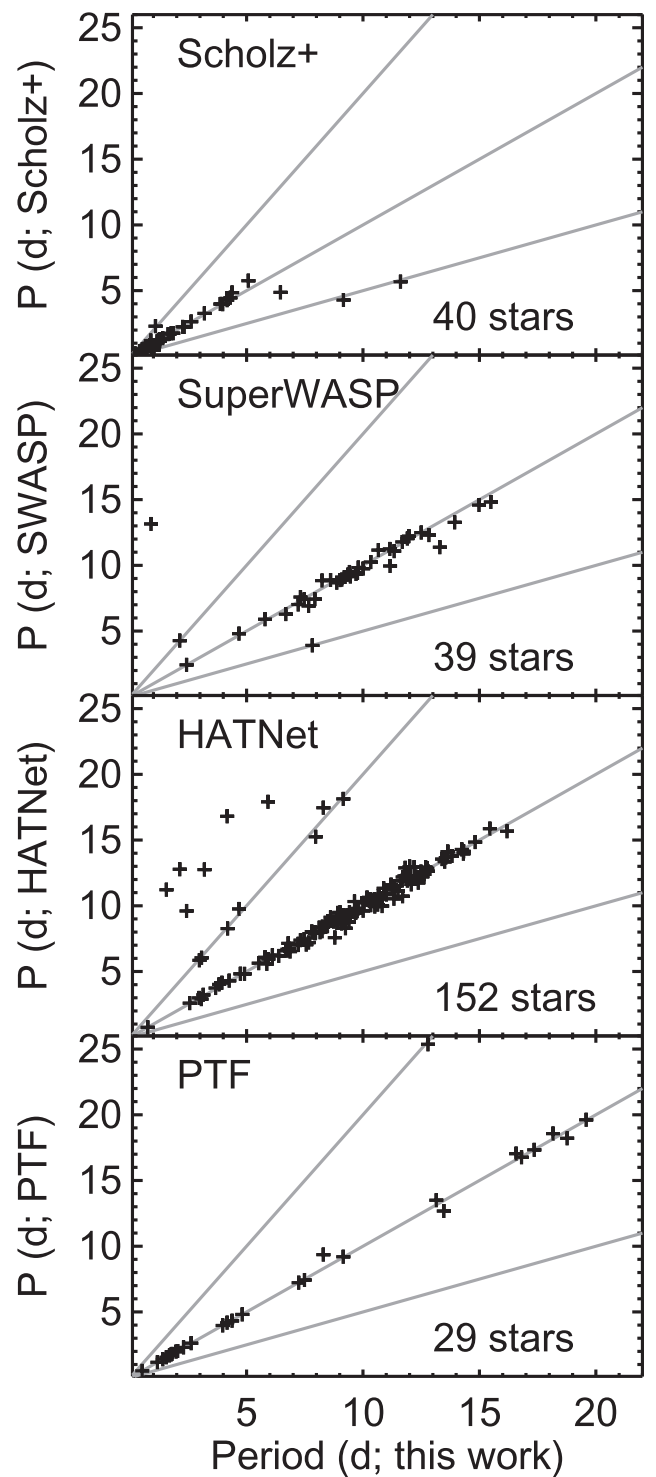

Figure 3. Comparison of periods obtained here to periods obtained in the literature. First panel: Scholz et al. (2011, 40 stars in common); second panel: SuperWASP (Delorme et al. 2011, 39 stars in common); third panel: HATNet (Kovács et al. 2014, 151 stars in common); fourth panel: PTF (Agüeros et al. 2011, 29 stars in common). The gray lines are at 1-to-1, $P / 2$, and $2 P$. Most of the periods match well (see the text).

Delorme et al. (2011) used SuperWASP to monitor 71 cluster members, looking for periods from 1.1 to 20 days, determining that 52 were periodic. Of these periodic stars, we have 39 in common (the remaining 13 fall in the gaps between $\mathrm{K} 2$ chips, or are otherwise off the K2 FOV). The periods derived for these 39 are shown in Figure 3. There is generally very good agreement; the median fractional difference $\left(P_{\text {SuperWAsp }}-P_{\text {herel }} / P_{\text {here }}\right)$ is $2.7 \%$. There are three stars for which our periods do not agree. One is EPIC 212013132/JS379 = 2MASSJ08404426+2028187, where it is likely that one of the surveys detected an harmonic. SuperWASP obtains a period of 4.27 days; we obtain three periods, including one that is close to 4.4 days, but the primary period we obtained for this star is 2.129 days. The second star is EPIC 211950227/2MASSJ08402554+1928328, where the K2 LC is very, very messy. SuperWASP obtains 13.15 days. The period we adopted for this one is 0.8984 days, and comes from 
only part of the LC, with most of the LC having been corrupted by instrumental effects. The third star is EPIC 211995288/ KW30 = 2MASSJ08372222+2010373, where we find a period of 7.8 days, a factor of 2 larger than the 3.9 days found in SuperWASP, so another harmonic. Having tested these periods by phasing our LCs at these alternate periods, we believe our periods to be correct in all cases for the epochs in which we observed these stars. There are no stars in common where SuperWASP has a period and we do not have a period.

Kovács et al. (2014) used HATNet (Hungarian Automated Telescope Networks) to monitor 381 members, finding 180 rotation periods, all ranging between 2.5 and 15 days. They identified 10 more stars as having what we would call "timescales," that is, repeated patterns that may or may not correspond to periods. There are 152 stars (150 out of their rotation periods and 2 more out of their "timescales") in common between the surveys, shown in Figure 3; most of the remaining stars fall in the gaps between chips, while a few could have been but simply were not observed. For the stars that we have in common, the median fractional difference $\left(\left|P_{\text {HATNet }}-P_{\text {here }}\right| / P_{\text {here }}\right)$ is $2.2 \%$. There are 13 stars for which the periods do not match, 7 of which are likely harmonics; we believe our period to be correct. One of the two from their "timescales" category is EPIC 211918335/HAT-269$0000582 / \mathrm{KW} 244=\mathrm{TX}$ Cnc, which Kovács et al. identify as an EB and Whelan et al. (1973) identifies it as a W UMa-type; we drop this $P_{\text {orb }}$. The other is 211947631/HAT-269-0000465/ BD $+19 d 2087$ (which Kovács et al. simply call "miscellaneous"); we retain $P=4.74$ days as a rotation period. There are no stars for which we both have LCs but HATNet has a period and we do not.

Agüeros et al. (2011) and Douglas et al. (2014) are a twopart study on the Palomar Transient Factory (PTF) observations of Praesepe. They used PTF to monitor 534 members, 40 of which were periodic between 0.52 and 35.85 days. There are 30 stars in common between the studies (see Figure 3), with the remaining 10 falling in the gaps between chips. The derived periods are generally in very good agreement; the median fractional difference $\left(\left|P_{\mathrm{PTF}}-P_{\text {here }}\right| / P_{\text {here }}\right)$ is just $0.36 \%$. There is one star (EPIC 211989299/AD2552 = 2MASSJ08392244 +2004548 ) for which one of us is likely to have found an harmonic, although the LC is messy (we obtain 12.791 and they obtain 25.36). We believe our periods to be correct for the epochs in which we observed these stars. For another one (EPIC 211886612/JS525), they found a period of 22.6 days; we retrieved an LS peak at 17.2 days for this object, but relegated it to "timescales" (in Appendix C; this is why only 29 stars appear in Figure 3). For the LC we have, it just does not look as nice as the rest of the clear $P_{\text {rot }}$ here.

We note here that, using K2, we find periods for a far higher number and higher fraction of Praesepe members than have been accomplished before. Moving to space enables higher precision and continuous photometry, resulting in a far higher fraction of detectable rotation periods.

\subsection{Membership and Supporting Data}

We started with the list of Praesepe members that we had proposed for this campaign and added targets from other related programs. From this list, we then amassed supporting data and assessed membership for each star. For some objects, we obtained additional Keck/HIRES spectroscopy; see Appendix I.
Table 1

Periods and Supporting Data for Praesepe Members with K2 Light Curves

\begin{tabular}{|c|c|}
\hline Label & Contents \\
\hline EPIC & Number in the Ecliptic Plane Input Catalog (EPIC) for K2 \\
\hline coord & R.A. and decl. (J2000) for target \\
\hline othername & Alternate name for target \\
\hline$V$ mag & $V$ magnitude (in Vega mags), if observed \\
\hline Kmag & $K_{\mathrm{s}}$ magnitude (in Vega mags), if observed \\
\hline vmk & $\begin{array}{l}\left(V-K_{\mathrm{s}}\right) \text {, as directly observed (if } V \text { and } K_{\mathrm{s}} \text { exist), or as } \\
\quad \text { inferred (see text) }\end{array}$ \\
\hline P1 & Primary period, in days (taken to be rotation period) \\
\hline $\mathrm{P} 2$ & Secondary period, in days \\
\hline P3 & Tertiary period, in days \\
\hline P4 & Quaternary period, in days \\
\hline $\mathrm{LC}$ & LC used as "best"a \\
\hline single/multi-P & $\begin{array}{l}\text { Indicator of whether single or multi-period star; if object has } \\
\text { a "timescale" (see Appendix C), that is indicated }\end{array}$ \\
\hline dd & Indicator of whether or not it is a double-dip LC \\
\hline ddmoving & Indicator of whether or not it is a moving double-dip LC \\
\hline shch & Indicator of whether or not it is a shape changer \\
\hline beat & Indicator of whether or not the full LC has beating visible \\
\hline cpeak & $\begin{array}{l}\text { Indicator of whether or not the power spectrum has a com- } \\
\text { plex, structured peak and/or has a wide peak }\end{array}$ \\
\hline resclose & $\begin{array}{l}\text { Indicator of whether or not there are resolved close periods } \\
\text { in the power spectrum }\end{array}$ \\
\hline resdist & $\begin{array}{l}\text { Indicator of whether or not there are resolved distant periods } \\
\text { in the power spectrum }\end{array}$ \\
\hline pulsator & $\begin{array}{l}\text { Indicator of whether or not the power spectrum and period } \\
\text { suggest that this is a pulsator }\end{array}$ \\
\hline
\end{tabular}

Note.

${ }^{\mathrm{a}}$ LC1—PDC, from MAST; LC2—version following A. M. Cody et al. (2017, in preparation); LC3 - version following Aigrain et al. (2015); LC4-version reduced by Vanderburg \& Johnson (2014) and downloaded from MAST; LC5 — version reduced by EVEREST code (Luger et al. 2016) and downloaded from MAST.

(This table is available in its entirety in machine-readable form.)

Table 1 includes the supporting photometric data we discuss in this section, plus the periods we derive here (in Section 2.2), for members of Praesepe. For completeness, non-members (NMs) appear in Appendix E.

\subsubsection{Metallicity, Reddening, Age}

Despite Praesepe's proximity and richness, its age and metallicity are a subject of considerable debate. Age estimates range from 600 to $900 \mathrm{Myr}$ : Harris (1976), $630 \mathrm{Myr}$; Mermilliod (1981), $660 \mathrm{Myr}$; vandenBerg \& Bridges (1984), 900 Myr; Janes \& Phelps (1994), 900 Myr; Bonatto et al. (2004), $729 \mathrm{Myr}$; Salaris et al. (2004), $700 \mathrm{Myr}$; and Brandt \& Huang (2015), 790 Myr. For this paper, we adopt the Brandt \& Huang age, both because it is based on the most recent physics and because it is a reasonable average of the other estimates. Metallicity estimates for Praesepe also span a somewhat surprisingly large range: Friel \& Boesgaard (1992), $[\mathrm{Fe} / \mathrm{H}]=+0.04 ; \quad$ An et al. (2007), $\quad[\mathrm{Fe} / \mathrm{H}]=+0.19$; Pace et al. (2008), $[\mathrm{Fe} / \mathrm{H}]=+0.27$; Boesgaard et al. (2013), $[\mathrm{Fe} / \mathrm{H}]=+0.12$; Blanco-Cuaresma et al. (2015), $[\mathrm{Fe} / \mathrm{H}]=0.20$; and Yang et al. $(2015),[\mathrm{Fe} / \mathrm{H}]=+0.16)$. We adopt $[\mathrm{Fe} / \mathrm{H}]=+0.15$ as a reasonable compromise value. The difference in metallicity should not matter significantly in the context of comparisons to other Kepler 
and K2 LCs, but we note it nonetheless as it might affect stellar structure and angular momentum evolution.

Despite the lack of agreement on age and metallicity, there is actually little dispute for the distance to the cluster, with modern values of $(m-M)_{0}=6.32$ (Salaris et al. 2004), 6.33 (An et al. 2007), and 6.30 (van Leeuwen 2009). We adopt $(m-M)_{0}=6.32$, corresponding to a distance of $184 \mathrm{pc}$.

The reddening to the cluster is quite small, though possibly as large as $E(B-V)=0.027$ (Taylor 2006). For convenience, we adopt $E(B-V)=0.0$. To the accuracy that we have determined $(\mathrm{V}-\mathrm{Ks})$, and to the accuracy that we need (V-Ks) for this analysis, the error from reddening is negligible.

In the subsequent analysis here, we often compare Praesepe results to those from the Pleiades (Rebull et al. 2016a, 2016b). We take the age of the Pleiades to be $125 \mathrm{Myr}$ (Stauffer et al. 1998), the distance to be $136 \mathrm{pc}$ (Melis et al. 2014), and the metallicity to be $[\mathrm{Fe} / \mathrm{H}]=+0.04$ (Soderblom et al. 2009).

\subsubsection{Target List}

We started with a list of members based on an in-house/ private open cluster database originally created by Prosser $\&$ Stauffer in the 1990s. For Praesepe, this was a merger of membership lists in Klein-Wassink (1927), Jones \& Cudworth (1983), and Jones \& Stauffer (1991), with some candidate members deleted due to discrepant photometry or radial velocities (RVs). This list was then merged with half a dozen recent proper motion membership studies (Adams et al. 2002; Kraus \& Hillenbrand 2007; Baker et al. 2010; Boudreault et al. 2012; Khalaj \& Baumgardt 2013; Wang et al. 2014), retaining stars considered as likely members in those papers. We then merged this Praesepe membership catalog with the list of all stars observed in K2 Campaign 5 within programs targeting Praesepe. About 600 did not have K2 LCs, sometimes due to the star falling in CCD gaps or just completely outside the $\mathrm{K} 2 \mathrm{FOV}$; in other cases, the star may have been observable, but no LC was obtained.

At this point, then, we have a set of 984 Praesepe members or candidate members with K2 LCs.

\subsubsection{Literature Photometry}

BVRI photometry on the Kron system has been published by Upgren et al. (1979), Weis (1981), Stauffer (1982), and Mermilliod et al. (1990). For stars not in those papers, we also queried the APASS database (Henden et al. 2016), particularly for $V$ magnitudes. We similarly queried the Gaia DR1 release (Gaia Collaboration et al. 2016) for their $G$ magnitudes (the released proper motions do not go faint enough for this work). Finally, we queried the recently released Pan-STARRS1 database (Chambers et al. 2016).

We added to this data from the Two-Micron All Sky Survey (2MASS; Skrutskie et al. 2006), from the Spitzer Space Telescope (Werner et al. 2004), including measurements from the Spitzer Enhanced Imaging Products, SEIP ${ }^{13}$, and from the Widefield Infrared Survey Explorer (Wright et al. 2010).

\subsubsection{Membership}

To refine the list of members, we queried the data from the United States Naval Observatory (USNO) Robotic Astrometric

\footnotetext{
$\overline{13}$ http://irsa.ipac.caltech.edu/data/SPITZER/Enhanced/SEIP/ overview.html
}

Table 2

Red Stars from the Pleiades and Hyades Used to Extend the Relation Between $V-K_{s}$ and $G-K_{s}$

\begin{tabular}{lcccl}
\hline \hline Star & $V(\mathrm{mag})$ & $G(\mathrm{mag})$ & $K_{s}(\mathrm{mag})$ & Source of $V$ \\
\hline HHJ3 & 22.14 & 18.78 & 14.13 & Stauffer et al. (1998) \\
HHJ5 & 20.71 & 18.45 & 13.93 & Stauffer et al. (1998) \\
HHJ8 & 20.60 & 18.38 & 14.23 & Stauffer et al. (1998) \\
HHJ10 & 20.74 & 18.39 & 13.77 & Stauffer et al. (1998) \\
PPL1 & 22.08 & 19.49 & 14.31 & Stauffer et al. (1989a) \\
PPL2 & 22.12 & 19.32 & 14.37 & Stauffer et al. (1989a) \\
PPL14 & 21.56 & 19.12 & 14.48 & Stauffer et al. (1994) \\
Bry804 & 19.27 & 16.83 & 12.37 & Bryja et al. (1994) \\
Bry816 & 18.83 & 16.61 & 12.20 & Bryja et al. (1994) \\
GJ512B & 13.70 & 12.10 & 8.30 & field star \\
\hline
\end{tabular}

Telescope (URAT; Zacharias et al. 2015) astrometric catalog to extract the " $f$ " magnitude (a broadband optical/red magnitude) and the URAT proper motions for these stars.

We then plotted the candidate Praesepe members in a vectorpoint diagram (VPD) and in two color-magnitude diagrams (CMDs), $f$ versus $f-K_{s}$ and $G$ versus $G-K_{s}$. In the VPD, we took the star as a member if the star's proper motion was within

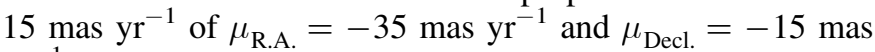
$\mathrm{yr}^{-1}$ (which is a mean motion consistent with the URAT proper motions for the cluster). For the two CMDs, we fit a polynomial curve to the single star locus and then took the star as a member if the star was displaced $<1.3$ magnitude above the locus or $0.7 \mathrm{mag}$ below the locus. For the stars included in our member catalog, we required that two of three of these flags (one flag for the VPD and one for each of the CMDs) be "true."

We removed stars flagged as non-members in the Mermilliod et al. (1990) RV and photometry survey for Praesepe halo members. For some objects identified as outliers in the analysis below, we obtained additional Keck/HIRES spectroscopy; see Appendix I. For those objects whose RVs were inconsistent with membership, we identified them as non-members (see Appendix E for a list).

From the initial sample of 984 LCs, then, 943 are members of Praesepe by our criteria (and 41 are non-members; see Appendix E).

\subsubsection{Obtaining $\left(V-K_{\mathrm{s}}\right)$}

Since we want to compare to the results we obtained in the Pleiades, we wanted to use $\left(V-K_{\mathrm{s}}\right)$ as a mass proxy in the same fashion as we did in the Pleiades.

We originally approached this the same way we did for the Pleiades, and used data from URAT. However, Gaia data are now available. Since $V-K_{s}$ is available (with $V$ and $K_{s}$ directly observed) for $\sim 250$ Praesepe stars with K2 LCs, we derived a formula to convert $G-K_{s}$ colors to $V-K_{s}$ colors by comparing the $V-K_{s}$ to the $G-K_{s}$. However, there are no observed $V$ photometry of known members for colors redder than about $V-K_{s}=5.5$. To extend the calibration of $G-K_{s}$, we have used faint Pleiades and Hyades stars (where $V$ photometry was obtained for very low-mass stellar and brown dwarf candidates in Pleiades and Hyades, respectively, by Stauffer et al. (1989a, 1994) and Bryja et al. $(1992,1994))$. To these, we added GJ512B, a field star. These stars are listed in 
Table 2. The final relationship between $V-K_{s}$ and $G-K_{s}$ is

$$
\begin{aligned}
\left(V-K_{\mathrm{s}}\right)= & 0.27354+0.7336 \times\left(G-K_{\mathrm{s}}\right)+0.1646 \\
& \times\left(G-K_{\mathrm{s}}\right)^{2}-0.000922 \times\left(G-K_{\mathrm{s}}\right)^{3} .
\end{aligned}
$$

We used this to interpolate $\left(V-K_{\mathrm{s}}\right)$ for those Praesepe stars lacking $\left(V-K_{\mathrm{s}}\right)$, but having a Gaia measurement. For about a dozen more stars, there is no Gaia measurement but there is a URAT measurement; we used the relationship we derived for the Pleiades to obtain a $\left(V-K_{\mathrm{s}}\right)$ estimate from the URAT data for these remaining stars.

All of the stars with a measured period have a measurement or estimate of $\left(V-K_{\mathrm{s}}\right)$; five of the non-periodic stars are missing a $\left(V-K_{\mathrm{s}}\right)$. For $\left(V-K_{\mathrm{s}}\right)<2.5$, median uncertainties (based on the scatter in the Gaia calibration above) are $\sim 0.017 \mathrm{mag}$; for $2.5<\left(V-K_{\mathrm{s}}\right)>5.5$, median uncertainties are $\sim 0.085$ mag.

\subsection{Bright and Faint Limits}

In the Pleiades, we discarded stars with $K_{\mathrm{s}} \lesssim 6$ and $K_{\mathrm{s}} \gtrsim 14.5$ as being too bright and faint, respectively, for the K2 LCs to be reliable. Here in Praesepe, the appropriate limits are less obvious; we have dropped the brightest $\left(K_{\mathrm{s}} \lesssim 6\right)$ and retained the rest.

There are two stars with $K_{\mathrm{s}}<6$, one of which we determined to be periodic, and both of which are listed in Appendix F. Both of them are discarded from our sample as too bright.

There are 21 stars with $6<K_{\mathrm{s}}<8 ; K_{\mathrm{s}}=8$ is roughly an F5 spectral type. At least 11 of them are likely pulsators (with 6 more likely pulsators that have fainter $K_{\mathrm{s}}$ ); see Appendix D. We have left these in the sample to allow for comparison to our Pleiades work (which also included likely pulsators), but have identified those pulsators where necessary in the remaining discussion.

In the Pleiades, there were very few sources with K2 LCs in the optical CMD below $K_{\mathrm{s}}=14.5$; as we will see below in Figure 4, such a cutoff is not as obvious here. There are many targets with $14.5<K_{\mathrm{s}}<15.5$ that have clear periods, and we have left those in the sample.

\subsection{Final Definition of Sample}

Final star counts are as follows. From an initial sample of 984 candidate members, we find 941 members that are also not too bright. Limiting it further, there are 809 stars that we find to be periodic in these $\mathrm{K} 2$ data.

Figure 4 shows color-magnitude diagrams for the stars detected as periodic and not detected as periodic. The periodic stars for the most part follow the expected main-sequence relation for Praesepe. The stars we do not detect as periodic appear to have a less well-defined main-sequence relation, which would be consistent with those stars more likely to be non-members, despite satisfying the membership criteria described in Section 2.5.4 above.

In the Pleiades, we determined $92 \%$ of the sample to be periodic (Rebull et al. 2016b); here, we obtain 809/941 $=86 \%$ of the member sample to be periodic. ${ }^{14}$ If we have more nonmembers inadvertently included in the sample for Praesepe than we did for the Pleiades (despite very similar selection

\footnotetext{
${ }^{14}$ The $84 \%$ in Section 2.3 refers to the fraction of the entire initial sample that is periodic; the $86 \%$ refers to the fraction of members that are periodic.
}

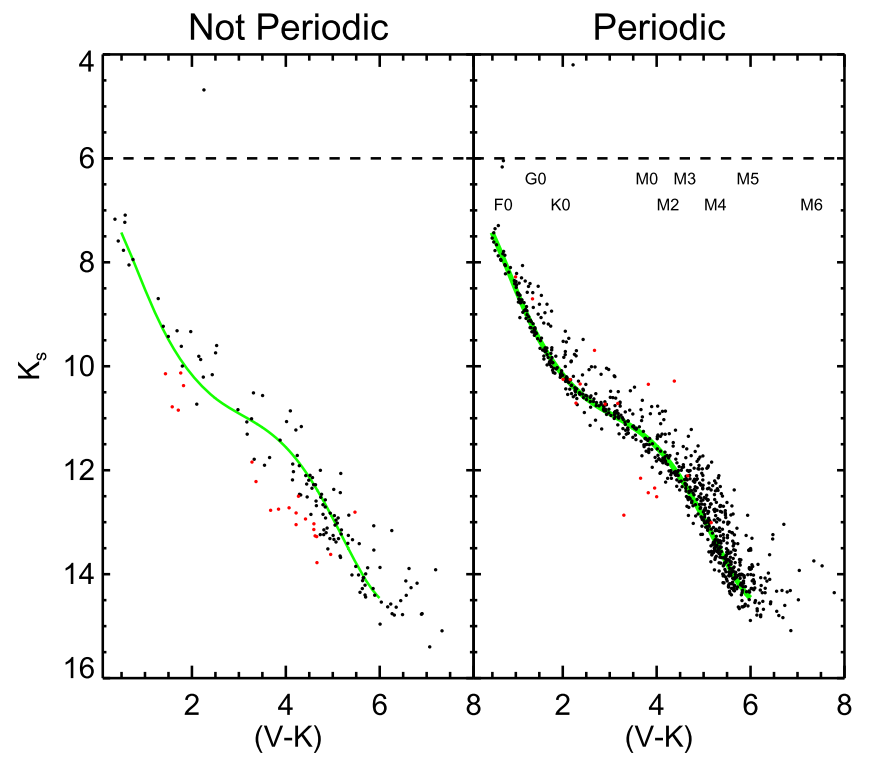

Figure 4. Color-magnitude diagram $\left(K_{\mathrm{S}}\right.$ vs. $\left.\left(V-K_{\mathrm{s}}\right)\right)$ for targets with $\mathrm{K} 2 \mathrm{LCs}$ and for which we had or could infer $\left(V-K_{\mathrm{s}}\right)$. The left panel shows stars not detected as periodic, and right panel shows stars for which we could measure periods. In both panels, red circles denote NM (see Section 2.5.4), and the green line is an empirical fit to the single star locus for Praesepe. Spectral types for a given $\left(V-K_{\mathrm{s}}\right)$ are as shown in the right panel. The periodic stars for the most part follow the expected main-sequence relation for Praesepe. The stars we do not detect as periodic appear to have a less well-defined main-sequence relation, which would be consistent with those stars being less likely members.

methods), a lower fraction of periodic stars might be expected. However, Praesepe is considerably older than the Pleiades, so the stars are expected to have fewer spots (hence lower amplitude signals) and rotate more slowly. Both of these factors would contribute to a lower fraction of detectably periodic members in Praesepe.

\section{Period and Period-Color Distributions}

Although many of the Praesepe stars, like many in the Pleiades, are multi-periodic (see Section 4 below), we have selected only one $P$ and color to be representative of the star in order to investigate the distribution of rotation rates. Now, we explore the overall distribution of $P$, and the distribution of $P$ as a function of $\left(V-K_{\mathrm{s}}\right)$ as a proxy for mass.

\subsection{Distribution of $P$}

The distribution of periods we found is shown in Figure 5; note that this excludes the stars with periods that were determined to be non-members (see Section 2.5.4 and Appendix E). The stars are rotating more slowly, on average, than the analogous figure from the Pleiades (see Figure 3 in Rebull et al. 2016b or Figure 9 in Rebull et al. 2016a). Whereas the Pleiades is strongly peaked at $<1$ day, only $\sim 20 \%$ of the Praesepe stars with rotation periods rotate faster than a day, with $\sim 42 \%$ rotating between 1 and 10 days, and $\sim 37 \%$ rotating between 10 and 35 days.

There seems to be a bimodal distribution of periods in Praesepe, with one peak near $\sim 1$ day and another peak near $\sim 10$ days. Assuming a Skumanich law (Skumanich 1972), $v_{\text {rot }} \propto t^{-0.5}$, and that the Pleiades is $125 \mathrm{Myr}$ and Praesepe is $800 \mathrm{Myr}$, we would expect the peak at $\sim 0.3$ days from the Pleiades to become the peak at $\sim 1$ day in Praesepe. This is the 


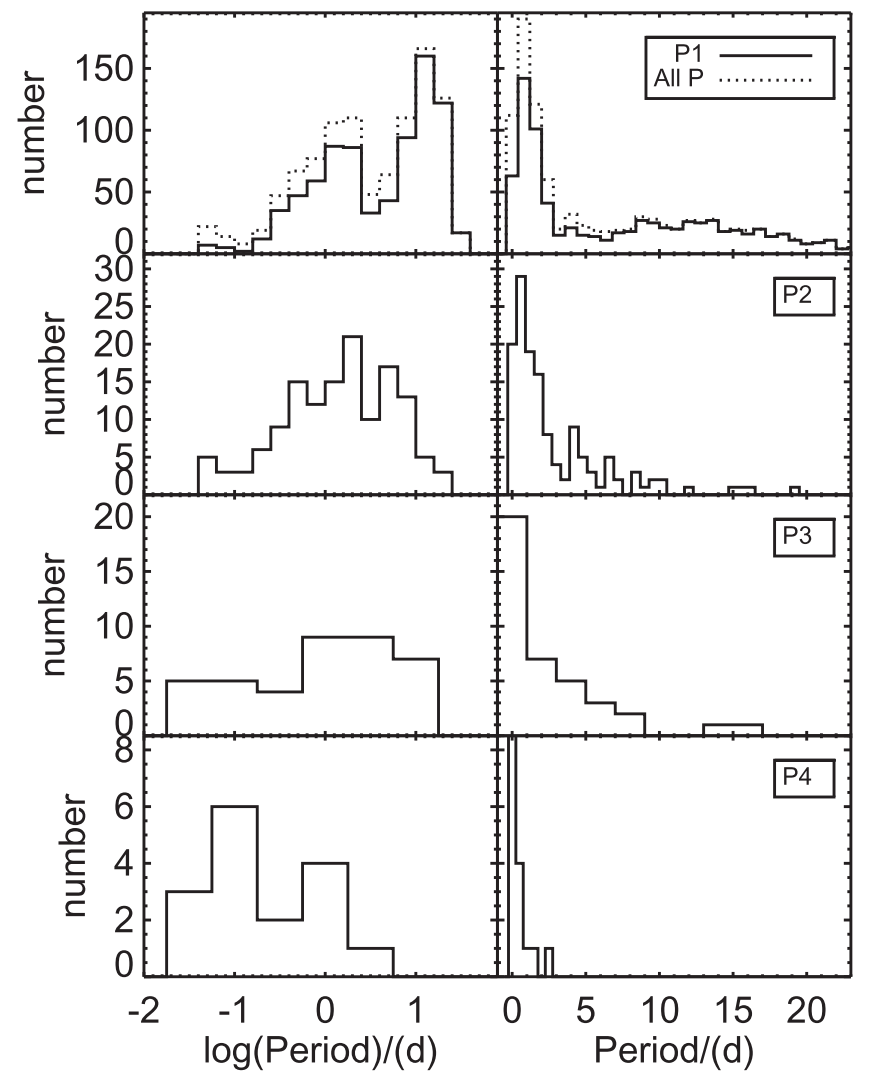

Figure 5. Histograms, on the left, of the log of periods, and on the right, of the linear periods, found by our analysis, in days. Solid line is the primary period (which which we take to be the rotation period of the star), and dotted line is (for reference) a histogram of all the periods found here, including the secondary, tertiary, and quaternary periods found here (see Section 4). There are two peaks, one near $\sim 1$ day and one near $\sim 10-15$ days. The faster peak corresponds largely to $\mathrm{M}$ stars, and the slower peak is primarily more massive stars. A significant fraction of the tertiary and quaternary periods are from likely pulsators.

case for the $\mathrm{M}$ stars, which compose most of the Pleiades peak at $\sim 0.3$ days and most of the Praesepe peak at $\sim 1$ day.

There is a substantial number of Praesepe stars, however, with periods near $10-15$ days, about $35 \%$ of the distribution. The stars contributing to this peak are for the most part more massive than those stars composing the other, faster rotating peak. Using the Skumanich law, these stars should correspond to Pleiades stars with periods of 4-6 days. Indeed, about $20 \%$ of the Pleiades stars with $\mathrm{K} 2$ rotation rates have periods greater than about 4 days; presumably, these go on to populate the slower peak in older clusters. However, there are fractionally more stars in the slower peak in Praesepe, which suggests that this peak includes stars with a larger range of masses than we have assumed in the rough Skumanich calculation.

Note that the 17 stars we have identified as likely pulsators are still in this sample shown in Figure 5; their periods do not make a significant difference to the histograms of $P_{1}$ (first period, e.g., the $P_{\text {rot }}$ ) or $P_{2}$ (secondary period), just because so many stars are represented in these plots. However, they do make a significant difference to the histograms of $P_{3}$ and $P_{4}$ (tertiary and quaternary periods), where they are largely responsible for the periods in these histograms.

\subsection{Morphology of $P$ versus $\left(V-K_{\mathrm{s}}\right)$}

Qualitatively, the morphology of the period versus color diagram for Praesepe, and the evolution of that distribution from young ages to Praesepe age, has been well-documented in the literature. The fact that by Praesepe's age G and K dwarfs have a very narrow distribution in period at a given mass was first shown for Hyades stars in the 1980s (Duncan et al. 1984; Radick et al. 1987). That F and early $G$ stars arrive on the main sequence with higher rotational velocities and subsequently spin down on the main sequence was first shown by Robert Kraft via spectroscopic rotational velocities for stars in a number of open clusters (Kraft 1967 and references therein). Later spectroscopic studies of those same clusters with more modern spectrographs and detectors showed that low-mass stars of all masses arrive on the main sequence with a wide range in rotational velocities and that angular momentum loss on the main sequence causes those stars to converge over time to a much narrower range in rotation at a given mass, with the convergence time being longer for lower masses (Stauffer \& Hartmann 1986; Stauffer et al. 1987, 1989b). The subsequent development of wide-format CCDs made it possible to obtain rotation periods for large samples of stars in open clusters, allowing the distribution of rotation rate as a function of mass to be determined for many of Kraft's open clusters (and other clusters), largely confirming the spectroscopic results but with better precision and larger samples of stars; see Gallet \& Bouvier (2015) and Coker et al. (2016) for a review of the rotation period data and theoretical models of angular momentum loss which attempt to explain the data. The LCs for open clusters and star-forming regions from $\mathrm{K} 2$ builds on this heritage, but also adds the benefits of photometric stability and signal-to-noise provided by space-based observations. For the open clusters observed with $\mathrm{K} 2$, these data for the first time allow the rotation periods of both stars in most binary systems to be determined, and for all cluster members observed, the LCs allow an assessment of the shape of the phased LC, which provides insight into the size and location of star spots on their surfaces.

Figure 6 shows the relationship between $P$ and $\left(V-K_{\mathrm{s}}\right)$ for Praesepe using K2 data. For comparison, Figure 6 also shows the $P$ versus $\left(V-K_{\mathrm{s}}\right.$ ) plot for the Pleiades $\mathrm{K} 2$ data. There have clearly been significant changes in these distributions between the ages of the Pleiades and Praesepe. As we noted above, Praesepe stars are on the whole rotating more slowly. However, the $\mathrm{M}$ stars in both clusters are primarily rapid rotators.

Figure 7 has the $P$ versus $\left(V-K_{\mathrm{s}}\right)$ plots again for both Praesepe and the Pleiades, but with color coding to aid in this discussion. In Praesepe, there are three relatively well-defined and apparently linear sequences. At the blue end (omitting the pulsators), for $(V-K)<1.3$ ( $\lesssim \mathrm{F} 8), \log P=1.748 \times$ $(V-K)-1.653$. $P$ is changing rapidly over a very small range of $\left(V-K_{\mathrm{s}}\right)$. There is an abrupt transition between the reddest end of this blue sequence and the bluest end of the next sequence; this is the Kraft break (Kraft 1967), where magnetic braking becomes less efficient for bluer stars. In the middle (yellow portion) of Figure $7,1.3 \leqslant(V-K)<4.5$ ( $\sim \mathrm{F} 8$ to $\sim \mathrm{M} 3), \log P=0.138 \times(V-K)+0.692$. In this regime, the points are tightly clumped around this relationship. For $4.5 \leqslant(V-K)<6.5, \sim \mathrm{M} 3$ to $\sim \mathrm{M} 6$ (which encompasses both the green and red points in Figure 7), $\log P=$ $-1.303 \times(V-K)+7.360$. Here again, $P$ is changing rapidly with $\left(V-K_{\mathrm{s}}\right)$. This end of the distribution contributes substantially to the bimodal nature of the $P$ distribution (Figure 5); there are many stars with $4.5 \leqslant(V-K)<6.5$ and relatively few stars with $2<P<10$. The transition between 

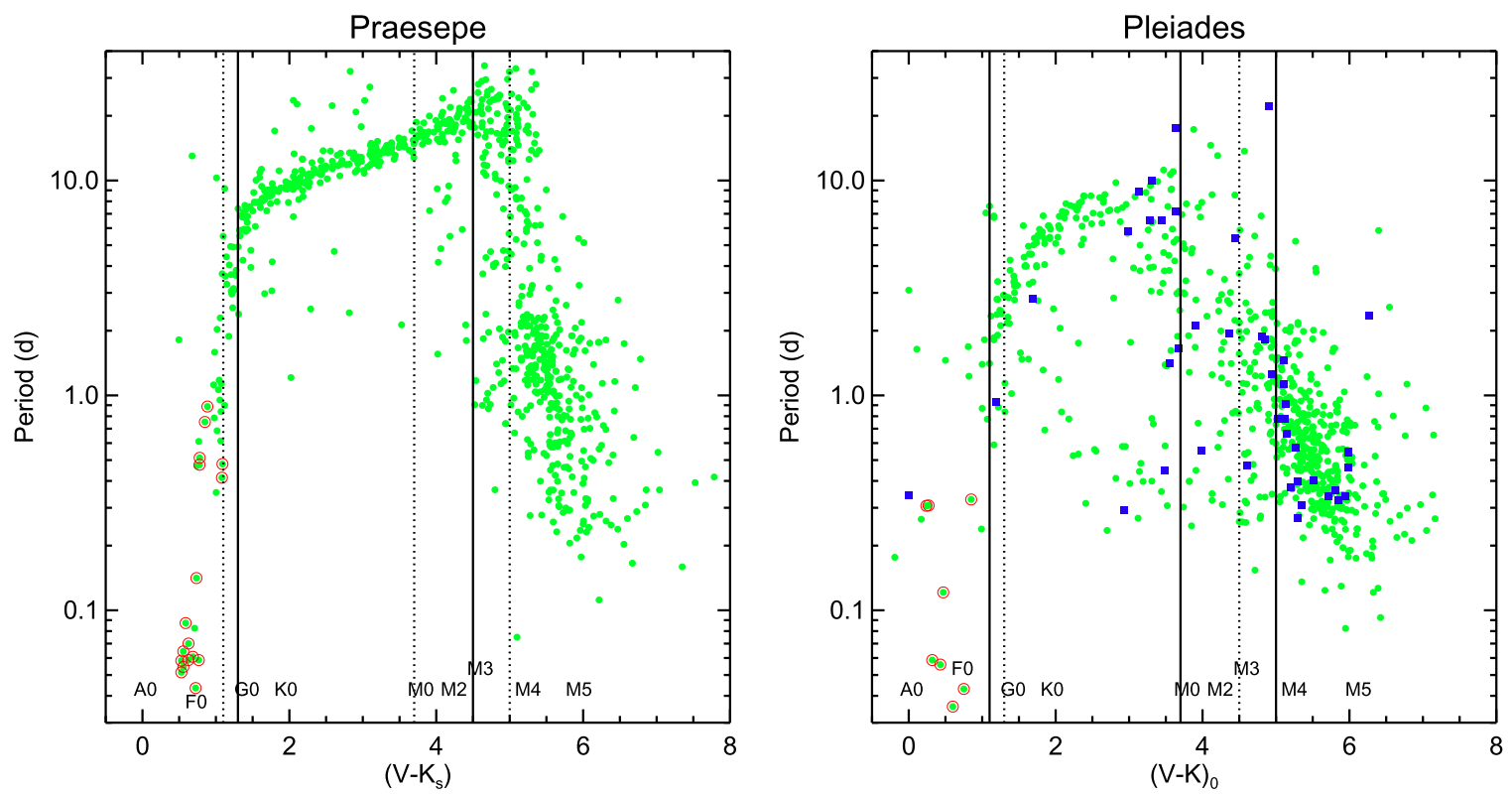

Figure 6. Plot of $P$ vs. $\left(V-K_{\mathrm{s}}\right.$ ) for Praesepe (left) and the Pleiades (right). The Pleiades plot is from Rebull et al. (2016b), their Figure 9. In the Pleiades, we had the best members (green dots) and the lower confidence members (blue squares). Likely pulsators (see Rebull et al. 2016a for the Pleiades) have an additional red circle in both panels. There is clearly considerable change between the age of the Pleiades and that of Praesepe. In each plot, the solid vertical lines denote different regions of the diagram defined in the discussion; the dotted vertical lines are the lines from the other cluster (the solid lines in the Praesepe plot are the dotted lines in the Pleiades plot and vice versa).
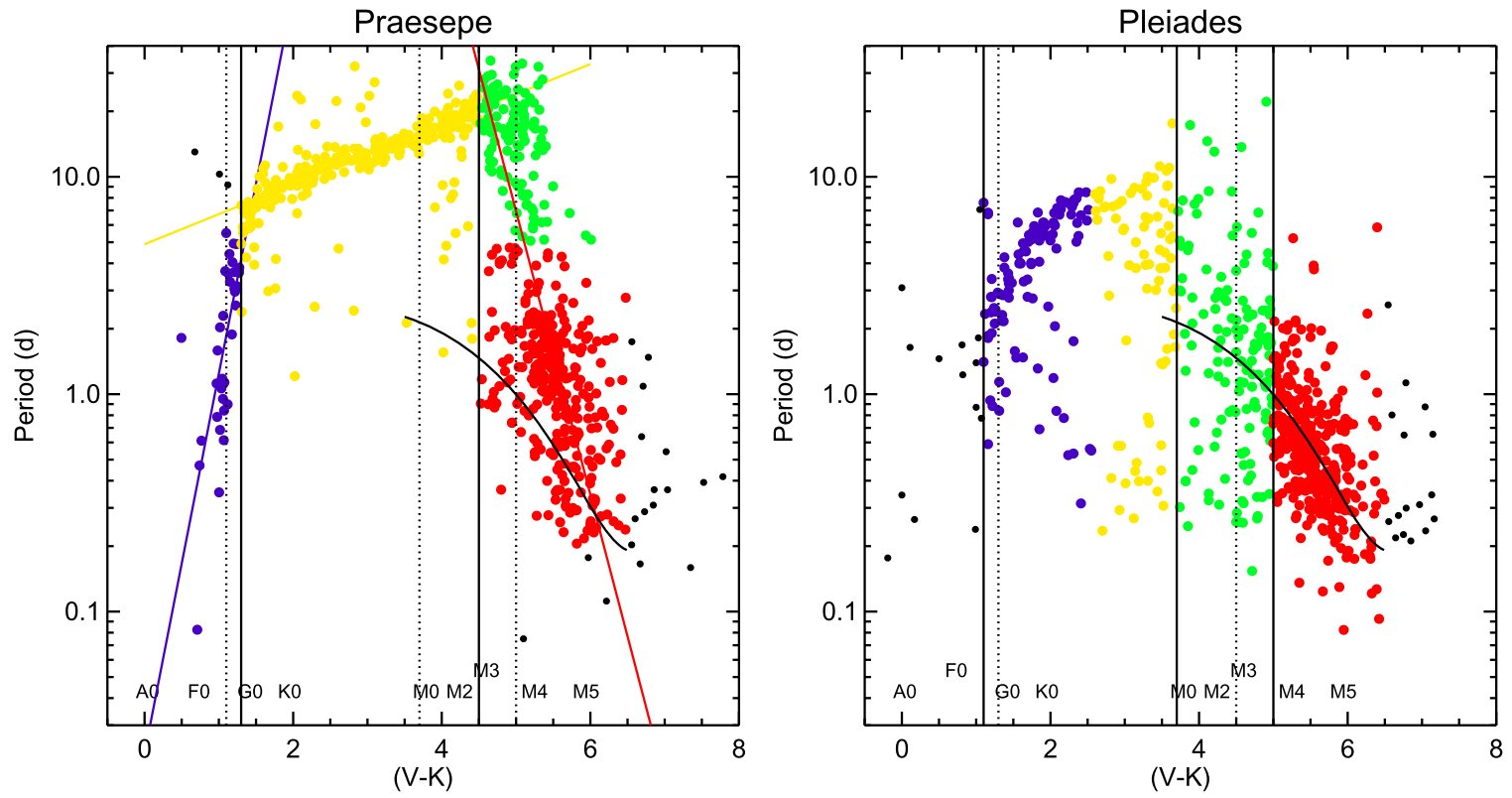

Figure 7. Plot of $\mathrm{P}$ vs. $\left(\mathrm{V}-\mathrm{K}_{\mathrm{s}}\right.$ ) for Praesepe (left) and the Pleiades (right), with additional color coding indicating regions discussed here and in Rebull et al. (2016b) and Stauffer et al. (2016). Obvious pulsators have been removed in both plots. Black points in both plots have been omitted as outliers for the purpose of fitting the lines. For Praesepe, colored points are as follows. Blue is $\left(\mathrm{V}-\mathrm{K}_{\mathrm{s}}\right)<1.3$; yellow is $1.3<\left(\mathrm{V}-\mathrm{K}_{\mathrm{s}}\right)<4.5$; green and red are both $\left(\mathrm{V}-\mathrm{K}_{\mathrm{s}}\right)>4.5$, but those with $\mathrm{P}>5$ are green and those with $\mathrm{P}<5$ are red. Linear fits are also shown in the blue, yellow, and red+green regimes. For the Pleiades, colored points follow Stauffer et al. (2016), Figure 24: blue is $1.1<(\mathrm{V}-\mathrm{Ks}) 0<2.6$ and yellow is $2.5<(\mathrm{V}-\mathrm{Ks}) 0<3.7$. The bulk of the distribution through both of these regions trace out the "slowly rotating sequence"; the transition to yellow is placed at the location of the "kink" in the slowly rotating sequence. Green is $3.7<\left(\mathrm{V}-\mathrm{K}_{\mathrm{s}}\right) 0<5$, the "disorganized region"; red is $\left(\mathrm{V}-\mathrm{K}_{\mathrm{s}}\right) 0>5$, the "fast sequence." The vertical lines are the same as the prior figure. The curved black line is the fit to the Pleiades $\mathrm{M}$ stars from Stauffer et al. (2016) and is the same in both panels. The M star relation is steeper in Praesepe.

the blue and yellow regions in Figure 7 is abrupt. The transition between the yellow and green regions is less obvious; the green points, at least the ones with $P>10$, could justifiably be included in the linear fit of the yellow points and the bulk of these points are already consistent with that fit. The green points, however, are also consistent with the relationship delineated by the red points (or even a slightly steeper relation). The end of the slow sequence (yellow through the green points consistent with the relationship) in Praesepe is $\left(V-K_{\mathrm{s}}\right) \sim 5.2$, or M4. 
Figure 7 also includes the Pleiades (Rebull et al. 2016b) for context. The color coding in Figure 7 follows Stauffer et al. (2016), Figure 24, and is not meant to trace exactly the same populations as seen in Praesepe, but simply illustrates different sections of the diagram that we call out in the text. In the Pleiades, we discussed the "slowly rotating sequence" for $1.1 \lesssim\left(V-K_{\mathrm{s}}\right) \lesssim 3.7\left(\sim \mathrm{F} 5\right.$ to $\sim \mathrm{K} 99^{15}, 2 \lesssim P \lesssim 11$ days), which is the blue and yellow points together in Figure 7 . The transition of blue to yellow is placed at the location of the "kink" in the slowly rotating sequence ( $\sim \mathrm{K} 3$; see Stauffer et al. 2016). The green points delineate a region in which there seems to be a "disorganized relationship" between $P$ and $\left(V-K_{\mathrm{s}}\right)$ between $3.7 \lesssim\left(V-K_{\mathrm{s}}\right) \lesssim 5.0(\sim \mathrm{K} 9$ to $\sim \mathrm{M} 3,0.2 \lesssim P \lesssim 15$ days). Finally, the red points represent the "fast sequence," with $\left(V-K_{\mathrm{s}}\right) \gtrsim 5.0$ ( $\gtrsim \mathrm{M} 3,0.1 \lesssim P \lesssim 2$ days).

Now between the Pleiades $(\sim 120 \mathrm{Myr})$ and Praesepe ( $~ 800 \mathrm{Myr})$, parts of this diagram see tremendous change (earlier types), and parts show more subtle changes (later types). All of the $\mathrm{G}$ and $\mathrm{K}$ (and even early $\mathrm{M}$ ) stars (yellow in the Praesepe plot) have spun down into a well-defined, linear relationship from the curved relationship (blue and yellow in the Pleiades plot). The "disorganized region" (green in the Pleiades plot, and even to some extent the yellow) is no longer quite so disorganized by Praesepe, where many of the earlier M stars have spun down into a relationship consistent with the $G$ and $\mathrm{K}$ stars. The Praesepe early-M stars are on average slow rotators, and the later $\mathrm{M}$ stars are on average more rapid rotators, but there is clear overlap between the rotation rates of early and late-M stars; one cannot divide the stars by mass and have them also divided by period. In the Pleiades, there is less obviously a bimodal distribution in $\mathrm{M}$ star periods, and (in contrast to Praesepe) the division between fast and slow rotators is also roughly a division in mass. The overall $\mathrm{M}$ star relationship (the red points in both panels in Figure 7) sees less obvious changes compared to the large changes for the more massive stars, but there are differences for the $\mathrm{M}$ stars, too. The slope of the M star relationship between period and color is overall much steeper in Praesepe than in the Pleiades; this is most easily seen from the black curved line in Figure 7, which is the relationship for Pleiades $M$ stars derived in Stauffer et al. (2016).

In the Pleiades, some of the $\mathrm{M}$ stars are still contracting (still spinning up); this is not the case in Praesepe. The M3 and M4 stars in Praesepe have spun down considerably, and they have a shorter contraction time than the M5 and later stars. The distribution of M5 stars has not changed much between the Pleiades and Praesepe. In the older cluster, angular momentum loss via wind braking has had more time to counteract the contraction in the M3-4 stars, and the balance between contraction/spin up and angular momentum loss must be different in the M5 (and later) stars.

The existence of a well-defined slow sequence of late $\mathrm{F}$ and even early G stars (blue points in Figure 7) in Praesepe presumably points to their having at least some amount of angular momentum loss. If they had no angular momentum loss at all, there would be a scatter in rotation, reflecting a range in initial angular momentum and a range in disk lifetimes. However, presumably their angular momentum loss rate is

\footnotetext{
15 Technically, some investigators have no K8 or K9 class defined; the dividing line here is based on linearly interpolating the $\left(V-K_{\mathrm{s}}\right)$ colors and the corresponding spectral type. This division might formally be at $\mathrm{K} 7$, but we use "K9" here to indicate succinctly "just before G0."
}

quite small since they have so little outer convective envelope. As a speculation, it is possible that the F dwarfs in Praesepe still have rapidly rotating radiative cores, with their observed rotation periods representing a balance between the angular momentum feeding up from below with the angular momentum lost from their winds. The $\mathrm{G} / \mathrm{K} / \mathrm{M}$ stars have much larger angular momentum loss rates, and have had time to spin down their cores. So, the two sequences reflect that dichotomy: core/ envelope still decoupled for the $\mathrm{F}$ dwarfs, core/envelope coupled for the $\mathrm{G}, \mathrm{K}$, and early Ms.

The bimodal period distribution of M stars in Praesepe (but not the Pleiades) is interesting because field $\mathrm{M}$ stars are found to have a bimodal $P$ distribution in the Kepler field (e.g., McQuillan et al. 2013; Davenport 2016) and in nearby M field stars (e.g., Kado-Fong et al. 2016; Newton et al. 2016). These field stars are older, on average, than Praesepe, and the locations of the two peaks are slower $(\sim 19$ and $\sim 33$ days $)$ compared to Praesepe with $\sim 1$ and $\sim 17$ days for the $\sim 500$ Praesepe stars with $\left(V-K_{\mathrm{s}}\right) \geqslant 3.79$, the color corresponding to M0. Assuming Skumanich evolution, these two peaks cannot both evolve together in lockstep. However, it is interesting that both distributions are bimodal. It may be that the mechanism that causes some M stars to rapidly spin down (e.g., Brown 2014; Newton et al. 2016) has started to operate in some of the Praesepe stars. We note, of course, that our data cannot constrain the behavior of M stars later than about M5 or M6.

\subsection{Outliers in $P$ versus $\left(V-K_{\mathrm{s}}\right)$}

Although the relationships delineated by the majority of the Praesepe stars in Figures 6 and 7 are striking, it is worth looking at some of the outliers in this distribution. Notes on specific stars appear in Appendix G, as well as optical color-magnitude and $P$ versus $\left(V-K_{\mathrm{s}}\right)$ diagrams with these objects highlighted.

The $\mathrm{G}$ and $\mathrm{K}$ stars in young open clusters like the Pleiades have bimodal rotational velocity distributions, with a majority of stars on a slowly rotating branch and a minority in a rapidly rotating branch. The latter stars are generally believed to descend from pre-main-sequence stars that lost their circumstellar disks (and hence their ability to rapidly shed angular momentum) very early. Our $P$ versus color plot also shows several rapidly rotating $\mathrm{G} / \mathrm{K}$ stars. Are these the descendants of the rapidly rotating $\mathrm{G} / \mathrm{K}$ stars in the Pleiades? For most or all of these stars, we believe not. Instead, they are best explained as tidally locked short-period binaries. Most of them are known short-period binaries; many of the remaining ones have little to no spectroscopic information, but tidally locked binaries are a logical explanation for stars that are rotating too quickly in comparison to other stars of similar colors.

The stars with periods much longer than average for their $\left(V-K_{\mathrm{s}}\right)$ color are also curious. For a few of them, tidal synchronization (or pseudo-synchronization) in a short-period binary may be the explanation, as has been advocated for similar outliers in M35 (Meibom et al. 2006) and other clusters. Because of the steep dependence of the synchronization time on the binary-star separation, for the longer period outliers ( $P>\sim 20$ days), this becomes a less viable possibility. If these stars are all Praesepe members, and if we are correct that all of them are rotation periods, then something about these stars made them spin down more than most other stars in the cluster. For these longest-period stars, interpretation of their LCs as rotation periods is more fraught than other shorter-period stars, 
Table 3

Star/Light Curve/Periodogram Categories

\begin{tabular}{|c|c|c|c|c|c|c|}
\hline \multirow{2}{*}{ Category } & \multicolumn{3}{|c|}{ Praesepe } & \multicolumn{3}{|c|}{ Pleiades } \\
\hline & Number & $\begin{array}{l}\text { Frac. of } \\
\text { Sample }\end{array}$ & $\begin{array}{c}\text { Frac. of } \\
\text { Periodic Sample }\end{array}$ & Number & $\begin{array}{l}\text { Frac. of } \\
\text { Sample }\end{array}$ & $\begin{array}{c}\text { Frac. of } \\
\text { Periodic Sample }\end{array}$ \\
\hline Periodic & 809 & 0.86 & 1.00 & 759 & 0.92 & 1.00 \\
\hline Single period & 645 & 0.69 & 0.80 & 559 & 0.68 & 0.74 \\
\hline Multi-period & 164 & 0.17 & 0.20 & 200 & 0.24 & 0.26 \\
\hline Double-dip & 163 & 0.17 & 0.20 & 107 & 0.13 & 0.14 \\
\hline Moving double-dip & 121 & 0.13 & 0.15 & 31 & 0.04 & 0.04 \\
\hline Shape changer & 297 & 0.32 & 0.37 & 114 & 0.14 & 0.15 \\
\hline Orbiting clouds? & 0 & 0 & 0 & 5 & $<0.01$ & $<0.01$ \\
\hline Beater & 77 & 0.08 & 0.10 & 135 & 0.16 & 0.18 \\
\hline Complex peak & 68 & 0.07 & 0.08 & 89 & 0.11 & 0.12 \\
\hline Resolved, close peaks & 68 & 0.07 & 0.08 & 126 & 0.15 & 0.17 \\
\hline Resolved, distant peaks & 71 & 0.08 & 0.09 & 39 & 0.05 & 0.05 \\
\hline Pulsator & 17 & 0.02 & 0.02 & 8 & 0.01 & 0.01 \\
\hline
\end{tabular}

as there is more variation from cycle to cycle, and it becomes more of a judgement call as to whether or not the star has a $P_{\text {rot }}$, or just a repeated pattern that may or may not be tied to rotation. Slowly rotating stars may undergo more significant spot evolution, so this may be a real astrophysical effect. Having fewer complete cycles within the K2 campaign, coupled with significant changes every cycle, makes it hard to assess. Many of the long- $P \mathrm{G}$ and $\mathrm{K}$ stars from the bulk of the distribution have more obviously sinusoidal LCs, and many of the longest $P \mathrm{M}$ stars (ones that are still with the bulk of the distribution) share LC characteristics with these long- $P$ outlier $\mathrm{G}$ and $\mathrm{K}$ stars; there is a continuum of LC properties such that it is not always easy to draw a line between $P_{\text {rot }}$ and just a repeated pattern.

Specifically because of this ambiguity, we obtained followup Keck/HIRES spectroscopy of many of these very slowly rotating stars (see Appendix I). Of the ones we observed, about half of them have RVs inconsistent with cluster membership. We identified these as non-members (Section 2.5.4 and Appendix E). Four of the longest $P$ objects (for which we have yet to obtain spectra) appear just below the single-star main sequence, suggesting that they may also be non-members; given the uncertainties in $\left(V-K_{\mathrm{s}}\right)$, we have provisionally left them in the list of members (see Appendix G).

\section{LC and Periodogram Categories}

\subsection{Identification of Categories}

In Rebull et al. (2016a), we presented a set of empirical structures in the K2 Pleiades LCs and periodograms, finding via visual inspection 11 different categories. These categories are discussed in detail in Rebull et al. (2016a), and we do not repeat that discussion here. All but one of the categories of LCs can be found in Praesepe as well; see Table 3 for a list. Many of the categories have more than one significant period. Figure 8 shows some examples of these classes in Praesepe, two of which have more than one measured $P$; these examples span a range of brightnesses, periods, and categories. (For examples of each of the categories in the Pleiades, see Rebull et al. 2016b.)

\subsection{Comparison to Pleiades}

Before we can compare the total counts of objects in the various periodogram categories, we need to be sure that we are sampling the same range of masses in the two clusters. Figure 9 shows the sample fraction for the periodic member samples as a function of $\left(V-K_{\mathrm{s}}\right)$ as a proxy for mass. The two samples are comparable over most of the range of $\left(V-K_{\mathrm{s}}\right)$; there are sample completeness effects at the reddest and bluest bins, which are the most poorly populated. The most notable differences are at the bluest end $\left(\left(V-K_{\mathrm{s}}\right) \lesssim 1\right)$, which will affect primarily stars whose measured $\mathrm{K} 2$ periods are most likely to be pulsation rather than rotation.

Now with the knowledge that the samples are of comparable mass ranges, we can compare sample fractions of LC/ periodogram categories. Table 3 summarizes the total counts of objects in each LC/periodogram category for Praesepe and the Pleiades (in both cases, only cluster members are included). There are some significant differences between the clusters. Overall, the fraction of stars that are singly periodic and multiply periodic are roughly comparable in the two clusters ( $\sim 75 \%-80 \%$ and $\sim 20 \%-25 \%$, respectively). However, there is a slightly higher fraction of singly periodic sources (and a slightly lower fraction of multi-periodic sources) in Praesepe.

In the context of this work, we identified an inconsistency in the way that we were identifying close and distant resolved peaks in our earlier Pleiades work. As stated there, we calculated $\Delta P / P_{1}$ (see also Section 6 below) and used that value to identify close $\left(\Delta P / P_{1}<0.45\right)$ and distant $\left(\Delta P / P_{1}>0.45\right)$ peaks. However, we went on to say (without explanation) that some objects could be identified as both close and distant peaks. We now more consistently identify an object as both close and distant peaks if the difference in any two periods divided by the $P_{\text {rot }}$ is $<0.45$ for two of at least three periods, and $>0.45$ for (a different) two of at least three periods (unless the star is already identified as a pulsator, in which case it is not identified as either close or distant peaks). As a result, three objects from the Pleiades that were already identified as resolved close peaks should also have been tagged as resolved distant peaks (EPIC 210877423, 211112974, and 211128979). This has been corrected in the statistics in Table 3 .

Among the LC/periodogram categories, there is a higher fraction of moving double-dip and shape changers in Praesepe 

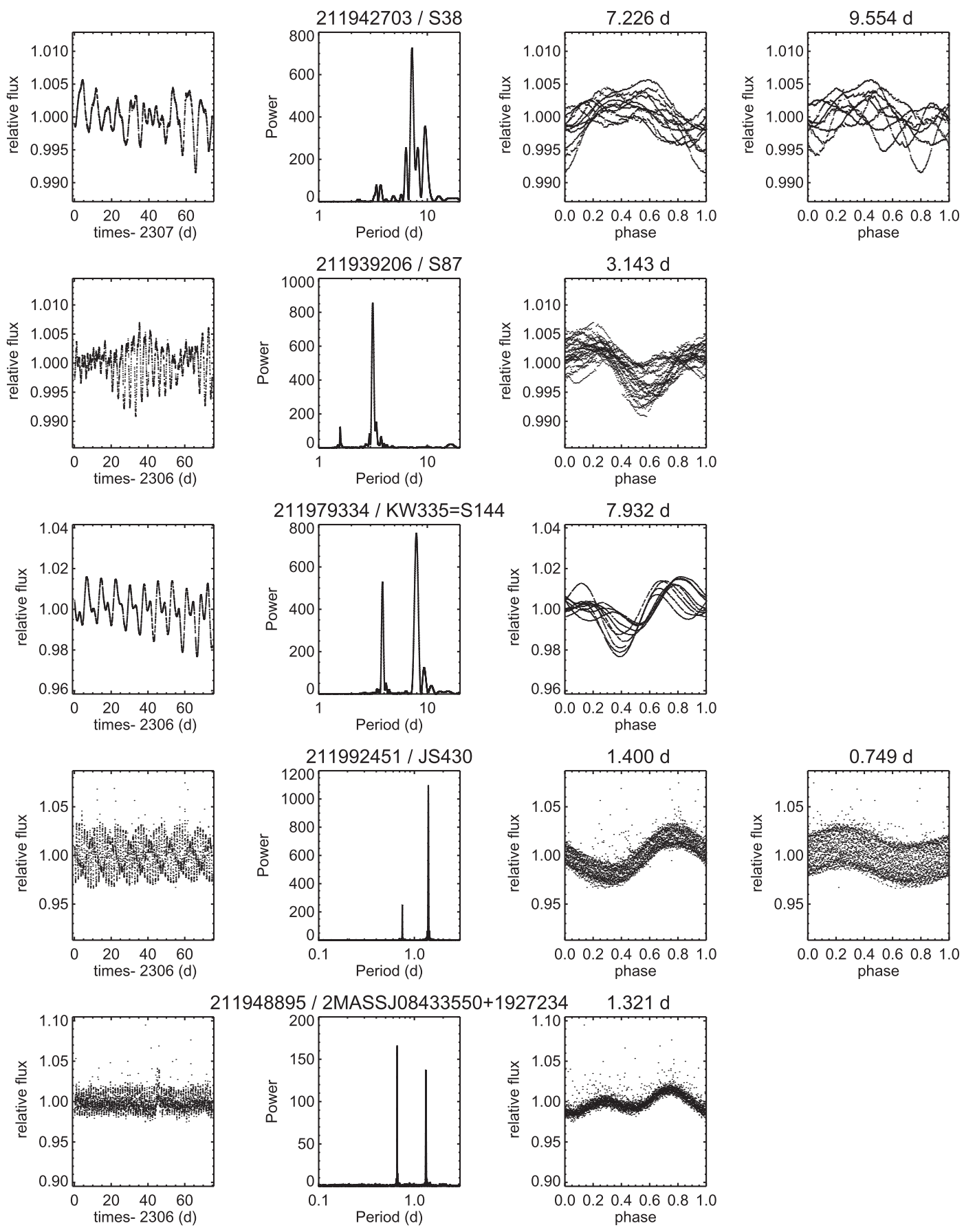

211948895 / 2MASSJ08433550+1927234 $1.321 \mathrm{~d}$
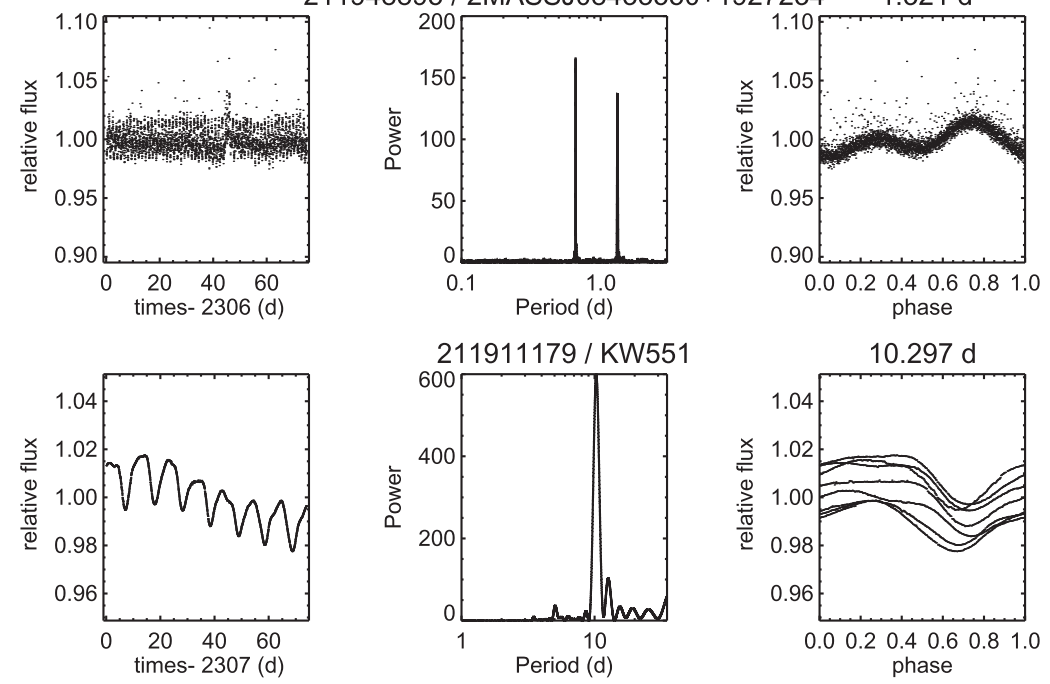

Figure 8. Six examples of LC/periodogram categories in the K2 Praesepe data. Left column: full LC; second column: LS periodogram; third column: phased LC, with best period (in days) as indicated; fourth column: phased LC to second period (if available, in days) as indicated. Rows, in order: $211942703 / \mathrm{S} 38$ (shape changer, beater, complex peak), 211939206/S87 (beater, complex peak), 211979334/KW335 = S144 (moving double dip [= double dip, shape changer]), 211992451/JS430 (beater, resolved distant peaks), 211948895/2MASSJ08433550+1927234 (double dip; appears to have two significant peaks in the periodogram, but really has only one real $P$ ), 211911179/KW551 (shape changer). These are representatives from a range of categories and periods. 


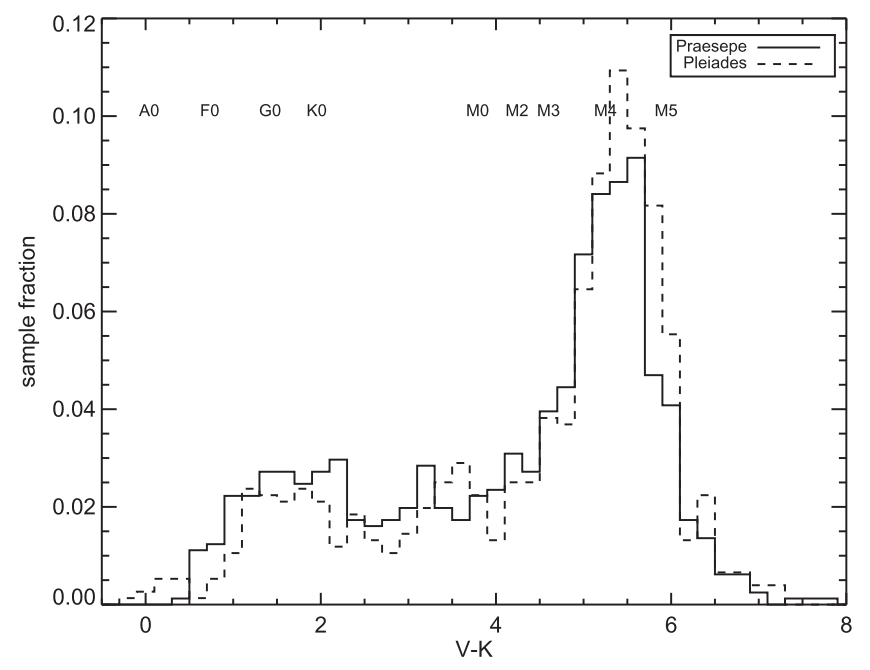

Figure 9. Distribution of $\left(V-K_{\mathrm{s}}\right)$ values for the member sample with rotation periods in both Praesepe (solid line) and the Pleiades (dotted line). The $y$-axis is in units of sample fraction. We are sampling the same range of $\left(V-K_{\mathrm{s}}\right)$ values overall in the two clusters; there are notable differences at the bluest and reddest ends.

than there are in the Pleiades; there are more than twice the fraction of shape changers as in the Pleiades ( $\sim 40 \%$ versus $\sim 15 \%$ ). In Rebull et al. (2016a) and Stauffer et al. (2016), we suggested that the shape changers and moving double dips are due to latitudinal differential rotation and/or spot/spot group evolution. These LC types were found primarily in the slower rotators in the Pleiades; with more slower rotators in Praesepe, it may not be surprising that we have more shape changers, because this may reflect a real difference in the incidence rate of differential rotation.

Because there is a slightly lower fraction of multi-period sources in Praesepe, there is a lower fraction of nearly all the multi-period subcategories in Praesepe. However, the most discrepant fractions are found in the resolved close peaks category, where the fraction is half what it was in the Pleiades. We postulated in Rebull et al. (2016a) and Stauffer et al. (2016) that the resolved peaks categories could be a result of latitudinal differential rotation and/or spot/spot group evolution among the $\mathrm{G}$ and $\mathrm{K}$ stars.

The shape changers, moving double dips, and close resolved peaks (in the $G$ and $K$ stars) may all be subject to an observational bias in the following sense. We noted in Rebull et al. (2016a) that, particularly for the shape changers/moving double dips, it was possible that if there had been much more data, $\gg 70$ days, encompassing more cycles, then it might have been possible to differentiate the two (or more?) periods contributing to the changing shape. In Praesepe, since the periods are on average longer than in the Pleiades, consequently there are (on average) fewer complete cycles encompassed in the $\mathrm{K} 2$ campaign. Thus, perhaps the higher occurrence rate of shape changers/moving double dips and the lower occurrence rate of resolved close peaks (for $\mathrm{G}$ and $\mathrm{K}$ stars) in Praesepe may be attributable to the number of complete cycles available for stars in Praesepe (as compared to the Pleiades).

For the M stars in the Pleiades, we postulated that the close resolved peaks were binaries, since most of them appeared above the single-star main sequence. The distant resolved peaks we thought were most likely to be binaries. The same basic result is true in Praesepe as well-the $M$ stars with resolved peaks are above the single-star main sequence (see Section 5.3 below). From theory, we expect stronger differential rotation in earlier stars (e.g., Kitchatinov \& Olemskoy 2012) and effectively solid-body rotation in the $\mathbf{M}$ stars. Praesepe has a comparable if not slightly greater fraction of resolved distant peaks, though there are many more instances where the difference between the peaks is very large indeed ( $>6$ days; see Section 6 below).

\subsection{Unusual LC Shapes}

The phased LCs of most spotted stars are simple, showing a morphology that is more or less sinusoidal or one that has one or two broad "humps" or dips. That is as expected, because spots located at most positions on a stellar surface and when viewed from most vantage points will be visible for half or more of the rotation period. That means that most spots will have a contribution to the phased LC that spans $180^{\circ}$ or more in phase. Flux dips or other structures that cover less than $90^{\circ}$ in phase are very hard to produce with spots. The only way they can be produced is by placing the spot very near the upper or lower limb of the star. Because of limb darkening and geometric foreshortening, light from such a location contributes little to the total integrated brightness of the star; therefore, flux dips from such spots cannot yield LC features that are very deep.

In the Pleiades (Rebull et al. 2016a), we nevertheless identified six stars with short-duration flux dips (full width at zero intensity, FWZI, $<0.2$ in phase); one star had three such dips, another had two, and the remaining four had just one flux dip. The dips were to first order constant in shape and depth over the duration of the K2 campaign. All six stars were mid-M dwarfs with very short $(P<0.7$ days $)$ periods. We identified another 19 rapidly rotating, mid-to-late-M dwarfs in the $8 \mathrm{Myr}$ old Upper Sco association whose K2 phased LCs seemed to show more structure than could be explained by spots (Stauffer et al. 2017). We have attributed the LC structure to warm clouds of coronal gas orbiting the stars near their Keplerian co-rotation radius, following models by Jardine \& van Ballegooijen (2005) and Townsend \& Owocki (2005).

We have found no Praesepe stars with the same properties (short-duration flux dips or other structure, rapid rotation, midto-late-M dwarf spectral type). However, there are three slowly rotating Praesepe $\mathrm{M}$ dwarfs $\left(\left(V-K_{\mathrm{s}}\right) \sim 5\right)$ that do have more structured LCs than we expect; these are shown in Figure 10: 212011416/2MASSJ08330845+2026372, 211915940/JS208, and 211931651/AD3196 = CP Cnc. One (EPIC 212011416/ 2MASSJ08330845+2026372) has a nearby saturated column in the K2 data, but multiple LC versions obtain the same shape and (large!) depth of the features. Note that in the first two cases, the highest peak in the periodogram results in a phased LC that has more scatter than when phased at three times the peak value; this is similar to the double dips (see discussion in Rebull et al. 2016a). These stars have periods $\gtrsim 10 \times$ longer than the stars in Pleiades or USco.

We do not believe the physical mechanism producing these LC morphologies is the same as for the Pleiades and Upper Sco. Because the flux dips are broader than in the Pleiades and are generally low amplitude, it is (barely) possible that spots could be responsible. However, the full amplitude of one of them (EPIC 212011416) is about 4\%, which would be very difficult to produce using spots located near the limb of the visible hemisphere. 

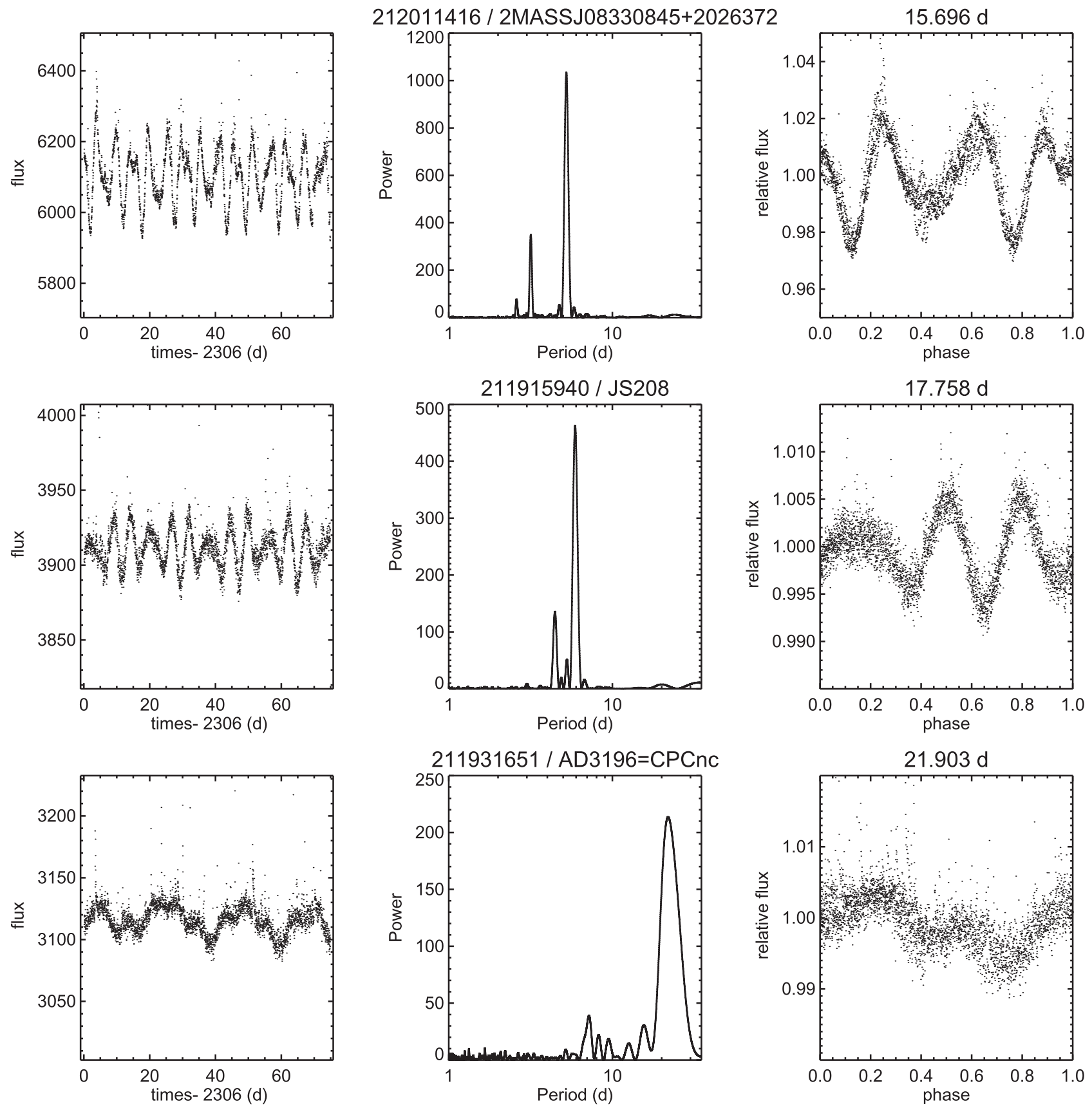

Figure 10. Three stars that have unusual shapes in their phased light curves. Columns: LC, periodogram, phased LC. Rows, in order: 212011416/2MASSJ08330845 +2026372 (note $x$-axis is just one phase, though it looks repeated), $211915940 /$ JS208, 211931651/AD3196 $=$ CP Cnc. These LCs all have structures in them uncharacteristic of most of the rest of the LCs in this study. They are all too slowly rotating to be of the unusual sorts found in the Pleiades or Upper Sco. See the text for additional discussion.

In order to help constrain the nature of these stars, we obtained single-epoch spectroscopy of these sources with Keck/HIRES (see Appendix I). All are consistent with being RV members of Praesepe and all are narrow-lined (and singlelined).

The phased LC morphologies for these three stars are much more complex than most of the rest of the LCs in Praesepe. Because they represent a tiny minority of the Praesepe sample, we do not believe that our failure to understand their properties should affect any of the other conclusions in the paper. We plan a future paper (Hebb et al.) that will discuss modeling of these LCs.

\subsection{Comparison to Other Classes in the Literature}

Kovács et al. (2014) also identified LC/power spectrum classes in their study of Praesepe. They had (a) monoperiodic sinusoids (13\% of their periodic sample); (b) single but unstable sinusoid (39\%); (c) two peaks interpreted as latitudinal differential rotation (7\%); (d) power moved into first harmonic $(41 \%)$.

(a) The monoperiodic sinusoids of their periodic sample can be matched to those in our sample with single periods but that are neither double dip nor shape changer. There are 366 of those, or $45 \%$ of the periodic sources, in our member sample. (b) Single but unstable sinusoids would be analogous to our 


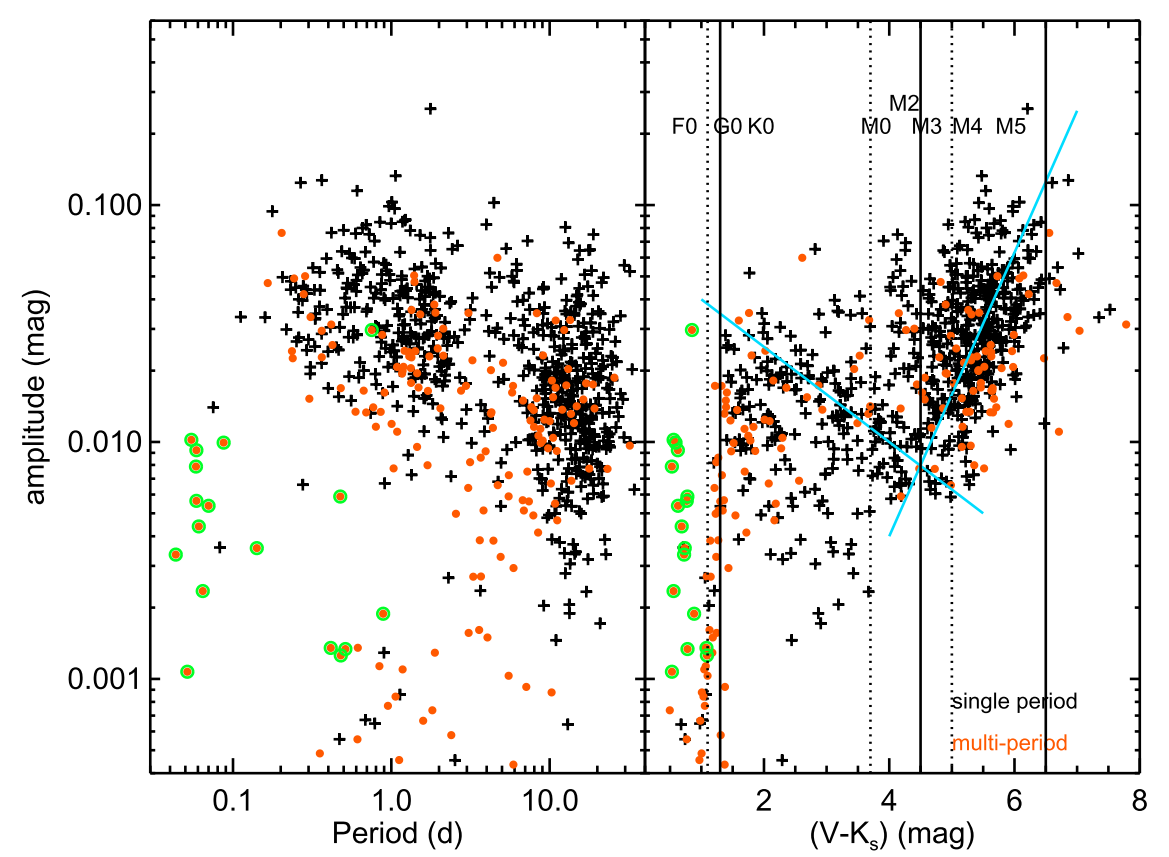

Figure 11. Amplitude (from the 10th to the 90th percentile), in magnitudes, of the periodic LCs, against $P$ (left) and $\left(V-K_{\mathrm{s}}\right)$ (right). The vertical lines are for the divisions from Figure 7-solid lines are for Praesepe and dotted lines are for the Pleiades. Black points are those stars with single periods, and orange dots highlight those with multiple periods. An additional green circle highlights the likely pulsators. Blue lines in the second panel are lines just to guide the eye in the text discussion. Longer periods have smaller amplitudes.

shape changers, without the double dips; we find 164 of them, $20 \%$ of the periodic sources. (c) They rarely find two discrete peaks in the power spectrum; this would be analogous to our resolved close and distant peaks combined, and we have 15\% of our periodic sample falling into this category. We agree that in some cases at least, this could be attributable to latitudinal differential rotation, but we suspect (also see discussion in Rebull et al. 2016a) that at least some (most of the multipeaked M stars) are binaries. (d) Lastly, their category where power is moved into the first harmonic is analogous to our double-dip category, which is $20 \%$ of our periodic sample.

Perhaps a more fair comparison is to work just with the 152 stars that are in common between the two studies. For that sample, we obtain (a) monoperiodic sinusoids: $6 \%$; (b) single unstable sinusoids: 23\%; (c) resolved peaks: 16\%; and (d) double dips: $47 \%$. The closest match in terms of sample fraction is this last category.

Assuming that we have correctly captured the relationship between our classes and those in Kovács et al. (2014), we have roughly similar sample fractions for the same characteristics.

\section{Comparison of the Single- and Multi-periodic Sources}

In the prior section, we identified the $\sim 20 \%$ of stars with multiple periods. In this section, we focus on where the multiperiodic sources fall with respect to the single-period sources in a variety of parameter spaces. We compare the single- and multi-period sources within Praesepe, and also to our analysis of the same phenomenon in the Pleiades (from Rebull et al. 2016a).

\subsection{Amplitudes}

We calculated the amplitude of the periodic signal in the same fashion as we did in the Pleiades; we assembled the distribution of all points in the LC, took the log of the 90th percentile flux, subtracted from that the $\log$ of the 10th percentile flux, and multiplied by 2.5 to convert to magnitudes. Figure 11 plots that amplitude against both $P$ and $\left(V-K_{\mathrm{s}}\right)$ for the periodic LCs. Note that this is not necessarily the amplitude of a sinusoid overlaid or fit to the periodic signal, but the amplitude of the overall LC, which necessarily includes longterm trends.

In the Pleiades, there was no obvious trend of amplitude with color or period. However, in Praesepe, there is a trend in both of these panels. On the left of Figure 11, longer period stars have lower amplitudes. This is consistent with expectations in that, certainly by this age, more slowly rotating stars should be less active and therefore have smaller spots. Note, however, that nearly all of the turn-down at the longest periods are higher-mass $\mathrm{G}$ and $\mathrm{K}$ stars; likewise, the shorter $P$ stars are primarily mid- to late-M stars. The bimodal distribution of periods seen in Figure 5 is apparent here, too. There are more multi-period low-amplitude sources than single-period lowamplitude sources (though several of these are likely pulsators, which explains the low amplitude in those bluest and smallest $P$ cases). On the whole, however, there is no clear distinction in the left panel of Figure 11 between the distributions of singly and multi-periodic sources.

On the right of Figure 11, it can be seen that bluer stars (corresponding to $\left(V-K_{\mathrm{s}}\right)<1.3$, blue points in Figure 7) are generally multi-periodic and have smaller amplitudes, not all of which are likely pulsators. There are several lower amplitude LCs with colors redder than $\left(V-K_{\mathrm{s}}\right) \sim 1.3$. This, and the trend toward larger amplitudes at even redder colors $\left(\left(V-K_{\mathrm{s}}\right)>4.5\right)$, could be an observational bias in that stars need to be "bright enough" for a period to be derivable; fainter stars need larger amplitudes to be seen as periodic, and brighter stars with smaller amplitudes are more easily detected. Note, 


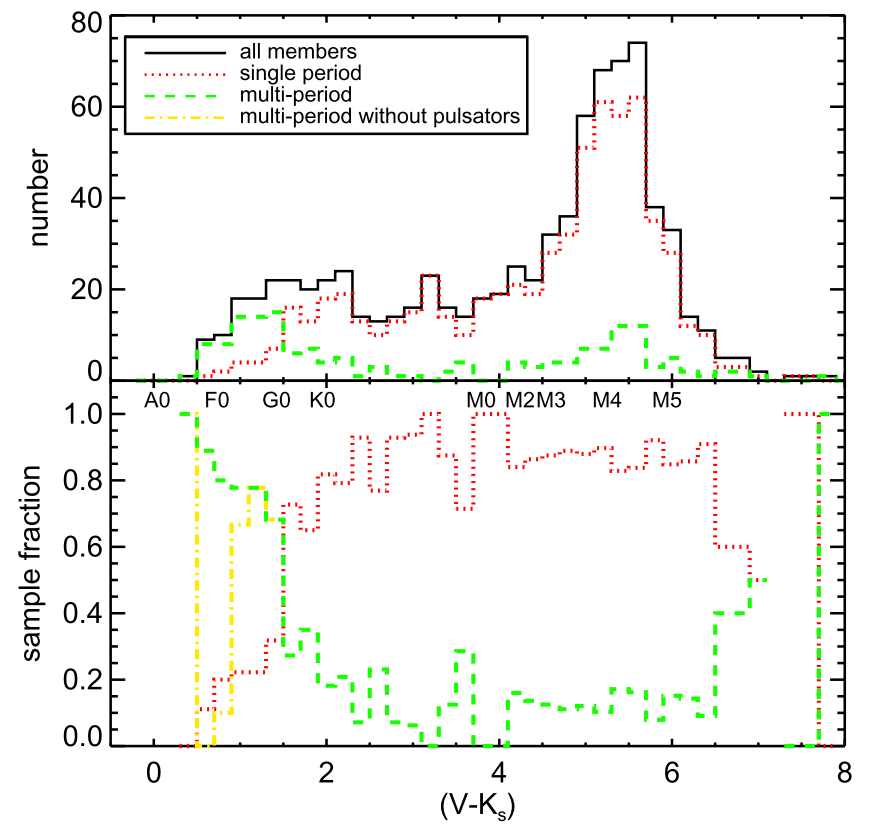

Figure 12. Distribution of $\left(V-K_{\mathrm{s}}\right)$ for the ensemble, with the single (red dotted line) and multiple (green dashed line) populations called out. The top panel is absolute numbers, and the bottom is the sample fraction. (The yellow dashed-dotted line is the sample fraction that is multi-periodic with the likely pulsators removed.) The transition between where multiple periods dominate is much bluer here $\left(\left(V-K_{\mathrm{s}}\right) \sim 1.5\right)$ than it was in the Pleiades $\left(\left(V-K_{\mathrm{s}}\right) \sim 2.6\right)$, and corresponds roughly to the point where the bluest "branch" turns down in the $P$ vs. $\left(V-K_{\mathrm{s}}\right)$ diagram in Figure 6 above. The fraction of the rest of the sample that has multiple periods is roughly constant with color through the rest of the sample.

however, that there is substructure within the right panel of Figure 11-there is a "clumping" of the distribution for $1.3 \leqslant\left(V-K_{\mathrm{s}}\right)<4.5$ (corresponding to the yellow points in Figure 7) that moves to lower amplitude as the color gets redder. Then, it turns around and moves to larger amplitude as the color gets redder for $\left(V-K_{\mathrm{s}}\right)>4.5$ (the green $/$ red points in Figure 7). For both of these color regimes, the amplitude gets smaller as the period gets longer. This makes sense in the standard rotation-activity sense, if the spot filling factor (at least for the non-axisymmetrically distributed component) decreases for longer periods.

\subsection{Distribution with Color}

In the Pleiades, we found a strong correlation between multiple periodicities and $\left(V-K_{\mathrm{s}}\right)$-most of the earlier stars were multiply periodic and nearly all the later stars were singly periodic. The distribution in Praesepe is different than in the Pleiades; see Figure 12. Through most of the sample, the fraction that has multiple periods is roughly constant with color. Clearly the bins for $\left(V-K_{\mathrm{s}}\right) \lesssim 1$ and $\left(V-K_{\mathrm{s}}\right) \gtrsim 6.5$ are significantly affected by sample completeness.

There is a transition between where multiple periods dominate to where single periods dominate, and it is at $\left(V-K_{\mathrm{s}}\right) \sim 1.5(\sim \mathrm{G} 0)$. The Pleiades sample extends to bluer colors, and the transition between where multiple periods dominate to where single periods dominate is at $\left(V-K_{\mathrm{s}}\right)_{0} \sim 2.6$ (early $\left.\mathrm{K}\right)$.

Inclusion of the likely pulsators in this analysis affects the results, particularly because of the sample differences for $\left(V-K_{\mathrm{s}}\right) \lesssim 1$ (see Figure 9). Dropping the likely pulsators

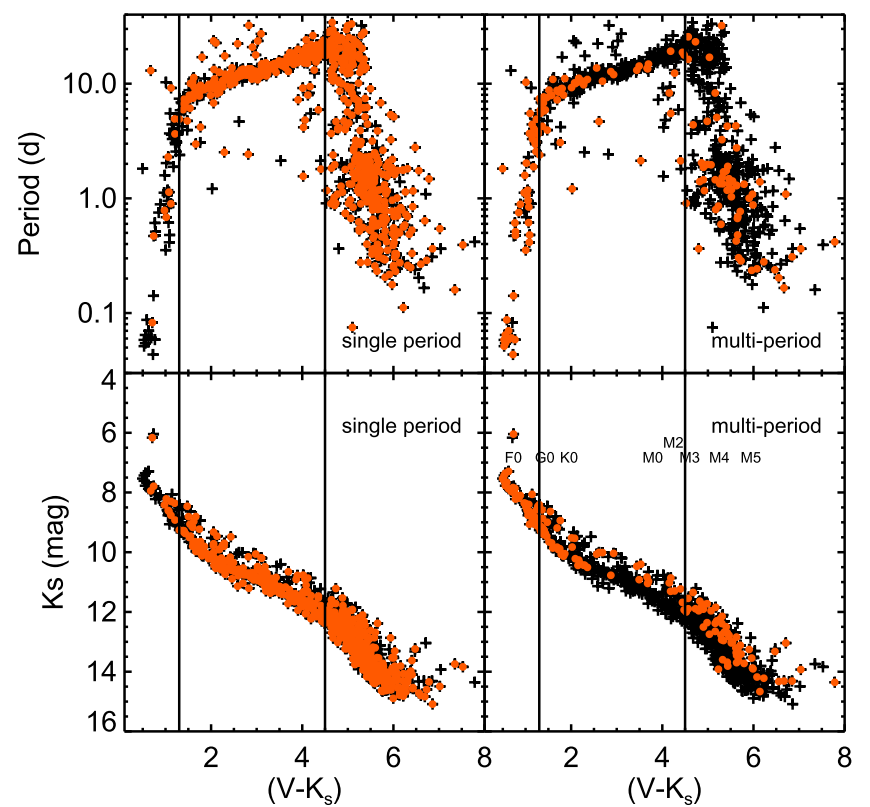

Figure 13. Plot of $P$ vs. $\left(V-K_{\mathrm{s}}\right)$ (top row) and $K_{\mathrm{s}}$ vs. $\left(V-K_{\mathrm{s}}\right)$ (bottom row) highlighting the single-period (left) and multi-period (right) populations. The vertical lines are at $\left(V-K_{\mathrm{s}}\right)=1.3$ and 4.5, the divisions are from Figure 7 above, for reference. The multi-period stars dominate for $\left(V-K_{\mathrm{s}}\right) \lesssim 1.5-2$. The $\mathrm{M}$ stars that are multi-periodic are more likely to be photometric binaries, as in the Pleiades.

causes the sample fraction of multi-period sources to plummet blueward of $\left(V-K_{\mathrm{s}}\right) \sim 1$. However, the multi-period sources still dominate for $1 \lesssim\left(V-K_{\mathrm{s}}\right) \lesssim 1.5$.

If latitudinal differential rotation dominates blueward of this $\left(V-K_{\mathrm{s}}\right) \sim 1.5$ transition point (which was one of our hypotheses in the Pleiades for the transition point there), then this transition has moved to more massive types by the age of Praesepe. This transition is also essentially where the bluest "branch" turns down in the $P$ versus $\left(V-K_{\mathrm{s}}\right)$ diagram in Figure 6 above, again suggesting that something physically different happens blueward of this color. Stronger differential rotation is expected for hotter stars (e.g., Kitchatinov \& Olemskoy 2012), so this may be the dominant effect. However, identification of a star as multi-periodic at all may be limited by the number of complete cycles in the $\mathrm{K} 2$ campaign, as discussed in Section 4.2.

\section{3. $P$ versus $\left(V-K_{\mathrm{s}}\right)$}

Figure 13 shows where the multi-period stars fall in the $P$ versus $\left(V-K_{\mathrm{s}}\right)$ and $K_{\mathrm{s}}$ versus $\left(V-K_{\mathrm{s}}\right)$ parameter spaces. This figure is in direct analogy to Rebull et al. (2016a), their Figure 12; in the Pleiades, most of the early-type stars were multi-periodic, and the later types were nearly all singly periodic, and the multi-periodic later type stars were nearly all photometric binaries.

In Praesepe, the single-period stars are distributed more uniformly throughout the diagram, consistent with Figure 12. The earlier-type stars are dominantly multiply periodic for $\left(V-K_{\mathrm{s}}\right) \lesssim 1.5-2$, which is a smaller range than for the Pleiades, again consistent with Figure 12. It is still true that most of the multi-period stars with $\left(V-K_{\mathrm{s}}\right) \gtrsim 3.5-4$ seem to be dominated by photometric binaries, given the position in the $K_{\mathrm{s}}$ versus $\left(V-K_{\mathrm{s}}\right)$ diagram. This is consistent with the M stars still rotating as solid bodies at Praesepe's age. 

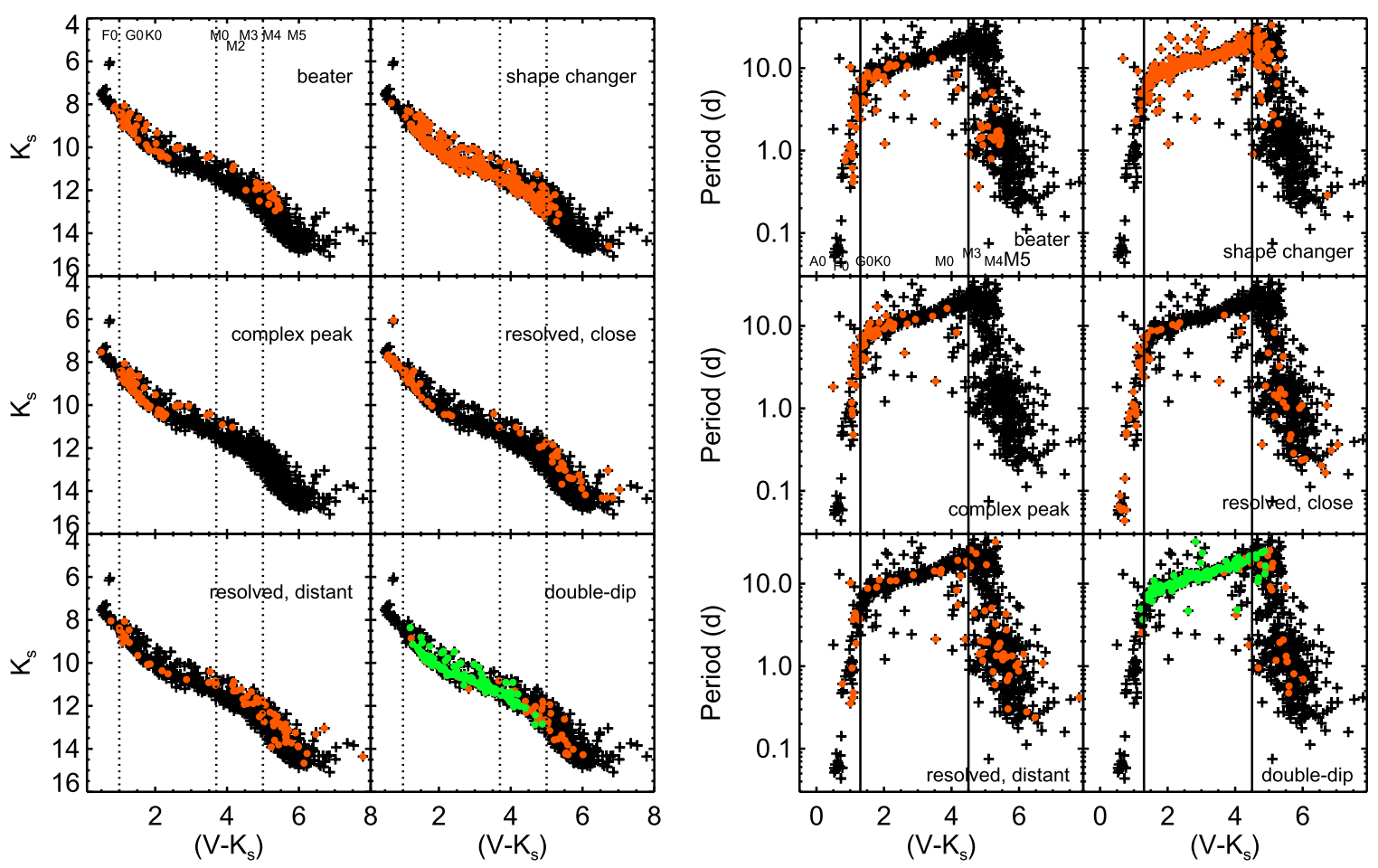

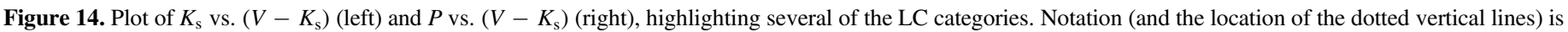
the same as in Figure 13, except for the double-dip panel, where the moving double-dip stars are green and the double-dip stars are orange.

The panels in Figure 14 break down where the individual LC classes appear in the $K_{\mathrm{S}}$ and $P$ versus $\left(V-K_{\mathrm{s}}\right)$ diagrams; the analogous Pleiades figures in Rebull et al. (2016a) are Figures 13 and 14.

Beaters and complex peaks were both, in the Pleiades, found to dominate the stars with $\left(V-K_{\mathrm{s}}\right)<3.7$. In Praesepe, the region where they dominate has shifted blueward, to $\left(V-K_{\mathrm{s}}\right) \lesssim 2.5-3$, consistent with the discussion associated with Figure 12 above. In both cases, beaters that are also M stars are more likely to be binaries than single stars, based on position in the CMD. Shape changers dominated in the Pleiades for $1.1<\left(V-K_{\mathrm{s}}\right)<3.7$, with a significant fraction of the stars in the "disorganized region" (with $3.7<\left(V-K_{\mathrm{s}}\right)<5$ ) being in this category. In Praesepe, there are a higher fraction of shape changers, and they dominate the "middle branch" (yellow points in Figure 7) with $1.3 \leqslant\left(V-K_{\mathrm{s}}\right)<4.5$. If the shape changers reflect rapid spot evolution and/or differential rotation, this happens more frequently in Praesepe than it does in the Pleiades. Note that the stars that exhibit shape-changing behavior in Praesepe are on average rotating much more slowly than in the Pleiades. The longer periods on the slowly rotating branch for $1.3<\left(V-K_{\mathrm{s}}\right)<4.5$ for Praesepe relative to Pleiades combined with the fixed campaign length for K2 makes it likely that stars will be moved from the resolved close peak category into the shape-changer or moving double-dip category, explaining at least some of the differences in LC class distributions we see.

The LCs with two distinct periods can be found throughout the $P$ versus $\left(V-K_{\mathrm{s}}\right)$ diagram for both clusters, and for $\mathrm{M}$ stars, tend to be on the brighter side of the cluster distribution on the CMD. For the M stars in particular, these are more likely to be binaries. Resolved distant peaks are distributed throughout the $P$ versus $\left(V-K_{\mathrm{s}}\right)$ diagram, but resolved close peaks tend to cluster in the bluest portion of the $P$ versus $\left(V-K_{\mathrm{s}}\right)$ diagram (and to some extent in the $M$ stars' fast-rotating clump). Resolved close peaks and bluer colors may be differential rotation and/or spot evolution. (See Section 6 below.)

Finally, moving double-dip stars dominate the "middle branch" (yellow points in Figure 7) of the Praesepe $P$ versus $\left(V-K_{\mathrm{s}}\right)$ diagram to a much larger extent than in the Pleiades. There are fractionally many more moving double-dip stars in Praesepe. There are fractionally far fewer $M$ stars exhibiting any double-dip behavior in Praesepe than in the Pleiades.

\section{6. $\Delta P$ Distributions}

Finally, we calculated the $\Delta P / P_{1}$ metric for stars with resolved multi-period peaks in Praesepe. In the Pleiades work (Rebull et al. 2016a), we took the closest two periods out of those detected, subtracted the smaller from the larger, and divided by the period we take to be the rotation period of the primary star in the system. In this calculation, it could be that the rotation period is not involved in the numerator. We have refined our thinking on this calculation, and now take the closest peak to $P_{\text {rot }}$, subtract the smaller from the larger, and divide by $P_{\text {rot }}$. This change ties the $\Delta P / P_{1}$ metric more closely to the rotation period of the star. Our previous calculation is effectively identical to this calculation for all but five stars in the Pleiades multi-period sample, though it affects $15 \%$ of the Praesepe stars with at least two periods (which is half of those with at least three periods.)

The plot of this corrected $\Delta P / P_{1}$ versus $P$ for Praesepe and the Pleiades is shown in Figure 15. (In both clusters, most of the likely pulsators are in a different region of the diagram than other stars.) The morphology of this diagram in Praesepe is 

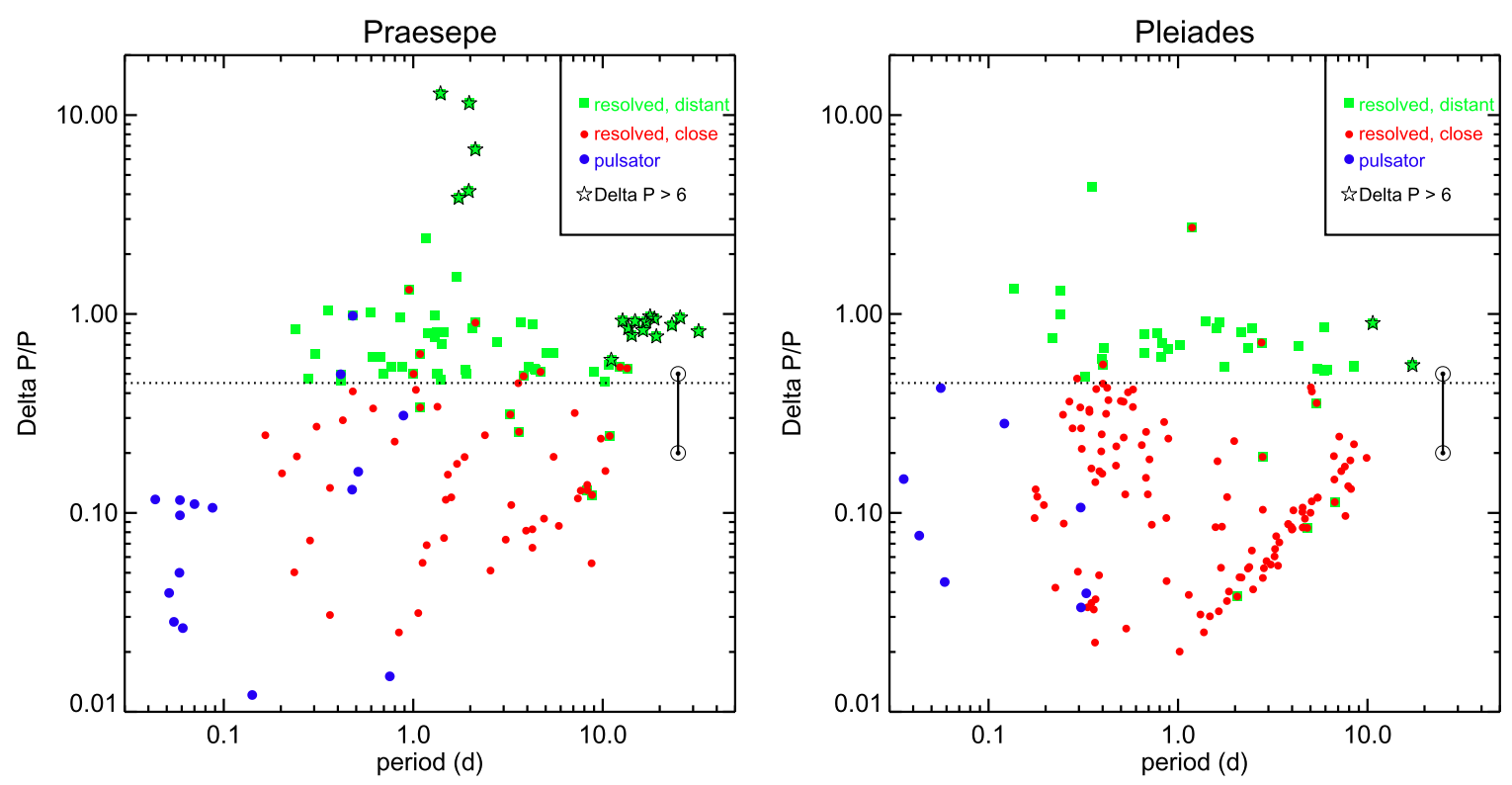

Figure 15. For Praesepe (left) and the Pleiades (right): plot of $\Delta P / P_{1}$ vs. $P$ for pulsators (blue dots), resolved distant peaks (green squares), and resolved close peaks (red dots). An additional black star indicates that $|\Delta P|>6$ days. The range of possible values for the Sun is included for reference $(\odot)$ ); if one takes as $\Delta P$ the range of periods measured where sunspots occur, $\Delta P / P_{1} \sim 0.1-0.2$, but if one takes the full range of $\Delta P$, equator to pole, $\Delta P / P_{1} \sim 0.5$. The dotted line is at $\Delta P / P_{1}=0.45$ and denotes the boundary between close and distant resolved peaks. The Pleiades data originally appeared in Figure 15 from Rebull et al. (2016a).

missing two prominent features from the Pleiades version of this figure. In the Pleiades, there was a very obvious linear feature in the lower right. Some of this was a selection effect in that points in the lower right are harder to observe (harder to distinguish periods and thus harder to obtain two periods that can be used to place them in this plot). In Praesepe, the feature is not as obvious. We suspected that in the Pleiades, this feature was a signature of differential rotation in stars primarily located on the slow sequence. As shown in Figure 13, Praesepe has very few multi-period stars on the slow sequence, and for the $\mathrm{K}$ and $\mathrm{M}$ dwarfs on that sequence, nearly all the multi-period stars appear to be binaries (based on their location on the CMD). As discussed in Section 4.2, the relative paucity of resolved close peak and moving double-dip stars on the slow sequence in Praesepe (the stars that primarily populate the linear sequence in the Pleiades $\Delta P / P$ versus $P$ plot) is likely a result of their longer periods-we simply do not have a long-enough sequence of data to resolve two periods in the periodogram.

The second prominent feature in Figure 15 that is present in the Pleiades but absent in Praesepe is a clump of stars near $P \sim 0.4$ and $\Delta P / P_{1} \sim 0.3$. Those points are primarily binary $\mathrm{M}$ dwarfs. We believe that the lack of a well-defined peak of binary $\mathrm{M}$ dwarfs in Praesepe is primarily a result of the dependence of angular momentum loss on mass and period. (See Figures 7 and 13.) If the Praesepe $M$ dwarf binaries were drawn from the same parent distribution at Pleiades' age, they would have started with very few stars with periods more than five days. Figure 7 shows that many of those stars must spin down to periods $>10$ days by Praesepe's age while others still maintain quite rapid rotation. Therefore, it is natural that the Praesepe $M$ dwarf binaries will have a much large range in period and a much larger range in $\Delta P / P_{1}$ than in the Pleiades.

Indeed, Praesepe has many more stars for which there are two very different periods. Those instances where $\Delta P$ is greater than six days are highlighted in the figure; they clump in two different locations depending on whether the shorter or the longer period was taken to be the $P_{\text {rot }}$ for the primary. (There are only two such points in the Pleiades with $\Delta P>6$.) None of these Praesepe $\Delta P>6$ points are particularly outliers in the $P$ versus $\left(V-K_{\mathrm{s}}\right)$ diagram; they are distributed between $1.8<\left(V-K_{\mathrm{s}}\right)<6.5$ within which the bulk of the distribution is located. The five stars near $P \sim 1, \Delta P / P_{1} \sim 3-20$ are in the $\mathrm{M}$ star fast-rotating sequence, and the remainder are within the top, slowly rotating sequence. They are not particularly clustered in the brighter portion of the cluster sequence in the $\mathrm{CMD}$, so they are not necessarily photometric binaries. However, it is hard to imagine a situation in which latitudinal differential rotation results in a $\Delta P$ of six days or more from pole to equator, and we suspect these are all binaries. The angular momentum loss mechanism operating in these stars is probably a function of both period and stellar mass. For the stars with $\left(V-K_{\mathrm{s}}\right) \gtrsim 4$, these could be examples of systems where one star has spun down and the other has not; the difference in periods in these binaries has significantly increased since the age of the Pleiades. These pairs could perhaps shed light on the mechanism that causes some M stars to rapidly spin down (see, e.g., Newton et al. 2016).

\section{Conclusions}

We have presented our analysis of nearly a thousand K2 LCs of Praesepe members, increasing the known Praesepe rotation periods by nearly a factor of four; 809 of the 941 members (86\%) with K2 LCs have a measured period from the K2 data. The distribution of $P$ versus $\left(V-K_{\mathrm{s}}\right)$, a proxy for mass, has three different regimes: $\left(V-K_{\mathrm{s}}\right)<1.3(\lesssim \mathrm{F} 8)$, where the rotation rate rapidly slows as the mass decreases; $1.3<\left(V-K_{\mathrm{s}}\right)<4.5(\sim \mathrm{F} 8$ to $\sim \mathrm{M} 3)$, where the rotation rate slows more gradually as the mass decreases; $\left(V-K_{\mathrm{s}}\right)>4.5$ ( $\gtrsim$ M3), where the rotation rate rapidly increases as the mass decreases. Particularly in this last regime, primarily the $\mathrm{M}$ star regime, there is a bimodal distribution of periods, with few between $\sim 2$ and $\sim 10$ days. We interpret this to mean that once 
M stars start to slow down, they do so rapidly; this is likely a predecessor of the bimodal distribution of $\mathrm{M}$ star rotation rates found in much older field stars (e.g., Kado-Fong et al. 2016; Newton et al. 2016).

The distribution of $P$ versus $\left(V-K_{\mathrm{s}}\right)$ exhibits significant changes between the Pleiades $(\sim 125 \mathrm{Myr})$ and Praesepe ( $\sim 790 \mathrm{Myr})$. For the late F, G, K, and early-M stars, the overall distribution slows considerably compared to the Pleiades, and the higher-mass branch (late $F$ and earliest $G$ ) steepens significantly. The transition at $\left(V-K_{\mathrm{s}}\right)=1.3$ is the Kraft break. The $\mathrm{G}$ and $\mathrm{K}$ and early-M stars have a more shallow relationship, with the lower masses rotating more slowly. For the mid-M stars, the period-color relationship changes relatively little between the Pleiades and Praesepe, though it is steeper in Praesepe than the Pleiades. Overall, these late-type stars rotate significantly faster as mass decreases, but there is more than an order of magnitude spread in the rotation rates in Praesepe at any given mass.

We found the same diversity of LC and periodogram classes in Praesepe as we did in the Pleiades. About three-quarters of the periodic member stars in both clusters have only one period; the rest have multiple periods. Praesepe has a higher fraction of LC classes that we have interpreted as latitudinal differential rotation and/or spot or spot group evolution, but this may be influenced by the observing window; Pleiades stars rotate faster, so there are more complete cycles observable in the $\sim 70$ day K2 campaign for the Pleiades stars than for Praesepe stars. Multi-periodic stars dominate the bluest end of the sample; the transition between where multi-period stars dominate over single-period stars happens at a bluer color in Praesepe $\left(\left(V-K_{\mathrm{s}}\right) \sim 1.5\right)$ than it does in the Pleiades $\left(\left(V-K_{\mathrm{s}}\right) \sim 2.6\right)$

$\mathrm{M}$ stars in both clusters that have multiple periods are likely to be binaries. In Praesepe, there are many more LCs that have two widely separated periods, $\Delta P>6$ days. Some of these could be examples of systems where one $M$ star has spun down but the other has not.

$\mathrm{K} 2$ continues to revolutionize the study of rotation in young and intermediate-age open clusters.

Some of the data presented in this paper were obtained from the Mikulski Archive for Space Telescopes (MAST). Support for MAST for non-HST data is provided by the NASA Office of Space Science via grant NNX09AF08G and by other grants and contracts. This paper includes data collected by the Kepler mission. Funding for the Kepler mission is provided by the NASA Science Mission directorate.

This research has made use of the NASA/IPAC Infrared Science Archive (IRSA), which is operated by the Jet Propulsion Laboratory, California Institute of Technology, under contract with the National Aeronautics and Space Administration. This research has made use of NASA's Astrophysics Data System (ADS) Abstract Service, and of the SIMBAD database, operated at CDS, Strasbourg, France. This research has made use of data products from the Two Micron All-Sky Survey (2MASS), which is a joint project of the University of Massachusetts and the Infrared Processing and Analysis Center, funded by the National Aeronautics and Space Administration and the National Science Foundation. The 2MASS data are served by the NASA/IPAC Infrared Science Archive, which is operated by the Jet Propulsion Laboratory, California Institute of Technology, under contract

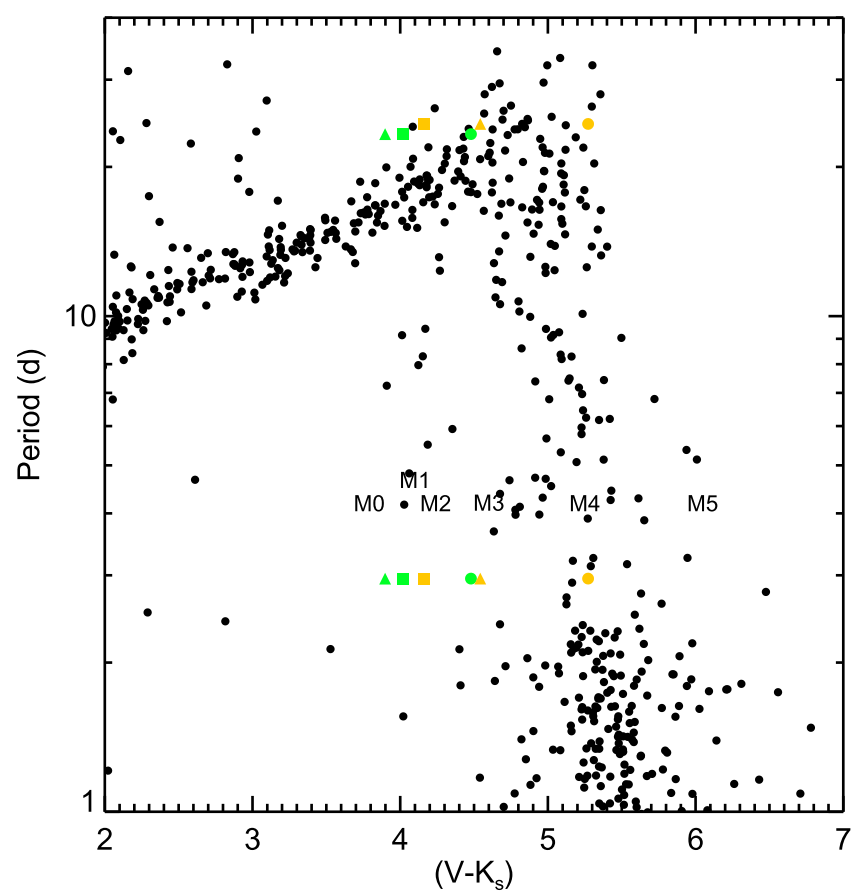

Figure 16. Zoom-in on the $P$ vs. $\left(V-K_{\mathrm{s}}\right)$ parameter space for the region in which EPIC 211934148/JS519 (green symbols) and EPIC 211934221/ UGCSJ084307.42+191419.2 (orange symbols) appear. (Black dots are the rest of the sample.) Approximate $\mathrm{M}$ spectral types are indicated as a function of $\left(V-K_{\mathrm{s}}\right)$ near the middle of the plot (in a relatively sparsely populated region). As described in the text, both periods appear in both LCs, one near 3 days and one near 24 days. Colored circles are using the $\left(V-K_{\mathrm{s}}\right)$ derived via Gaia magnitudes, triangles are using the $\left(V-K_{\mathrm{s}}\right)$ derived via URAT magnitudes, and squares are using the NOMAD-reported $V$ measurements (and 2MASS $K_{s}$ ) to obtain $\left(V-K_{\mathrm{s}}\right)$. We have retained the Gaia-based $\left(V-K_{\mathrm{s}}\right)$ estimates (circles) and assigned the $\sim 24$ day period to the bluer star (EPIC 211934148/ JS519, green circle) and the $\sim 3$ day period to the redder star (EPIC $211934221=$ UGCSJ084307.42+191419.2, orange circle).

with the National Aeronautics and Space Administration. This publication makes use of data products from the Wide-field Infrared Survey Explorer, which is a joint project of the University of California, Los Angeles, and the Jet Propulsion Laboratory/California Institute of Technology, funded by the National Aeronautics and Space Administration.

Facilities: Kepler, K2, 2MASS.

\section{Appendix A Two Close Stars}

Out of the 984 LCs, only one pair of stars for which a K2 LC was requested were within $4^{\prime \prime}$ of each other (within a Kepler pixel). EPIC 211934148 is at 08:43:07.40+19:14:15.4, and as such, is matched to JS519; EPIC 211934221 is at 08:43:07.44 $+19: 14: 19.2,3$, 88 away, and as such is matched to UGCSJ084307.42+191419.2. The two stars are of similar brightness in the POSS images, but the latter is slightly fainter.

Both of these stars are clearly $M$ stars. There are two obvious, effectively identical periods in each of the (very similar) LCs. The one from the brighter star (211934148) is about double the counts than the one from the fainter star (211934221). We suspect that each star is contributing one periodic signal. However, it is less clear which period belongs to which star.

The two periods derived from the joint LCs are very different from each other. A period of 2.954 days is recovered cleanly in both LCs. The other period, at $\sim 23-24$ days, is less reliably 

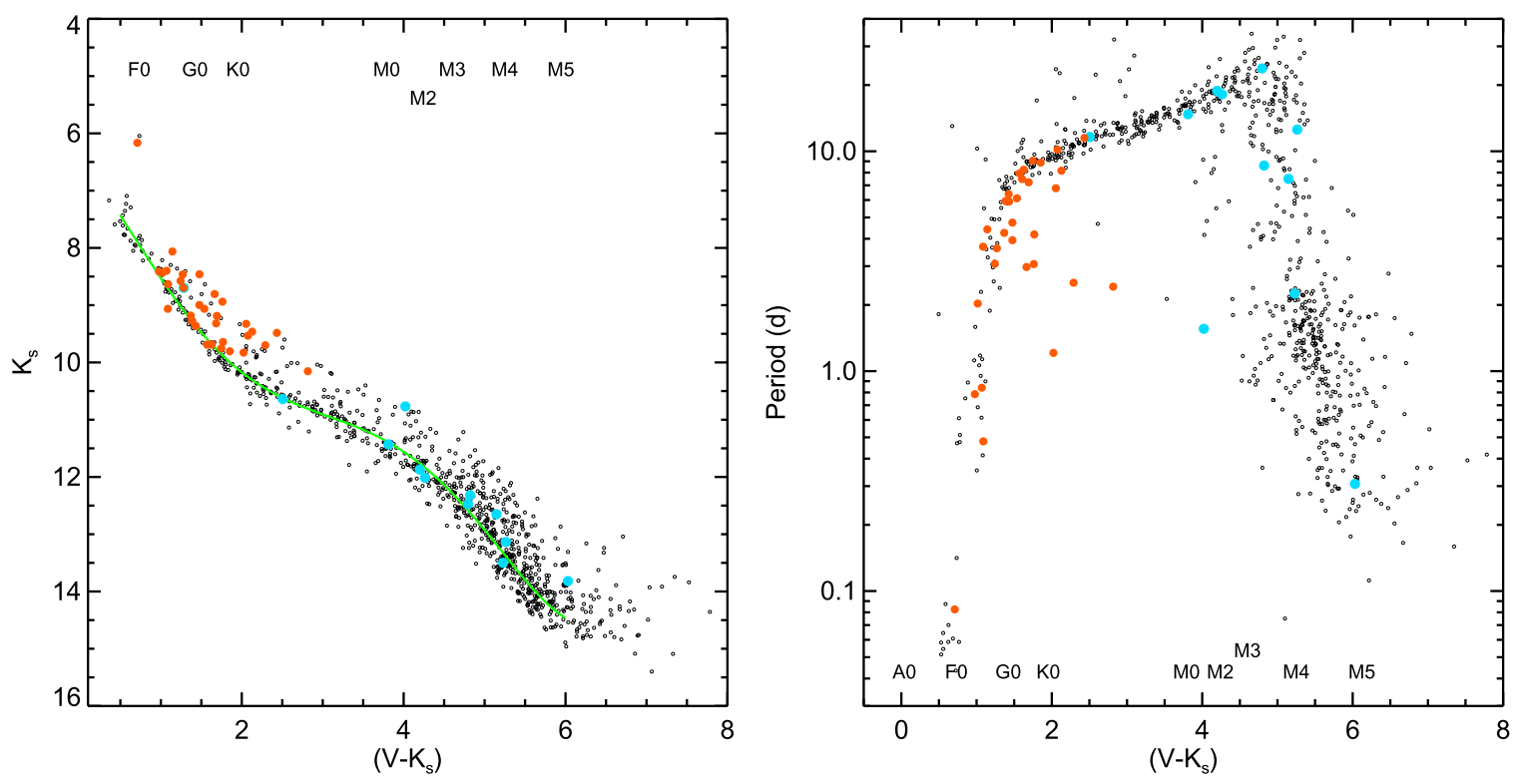

Figure 17. Optical CMD $\left(K_{\mathrm{s}}\right.$ vs. $\left.\left(V-K_{\mathrm{s}}\right)\right)$, left, and $P$ vs. $\left(V-K_{\mathrm{s}}\right)$, right, for the ensemble (small dots), stars with EBs or planets (orange dots), and stars that are literature binaries but for which we still have a rotation period (blue dots). Non-members have already been omitted. Many of the fast-rotating $\mathrm{G}$ and $\mathrm{K}$ outliers in the $P$ vs. $\left(V-K_{\mathrm{s}}\right)$ plot are known, tidally locked binaries.

Table 4

Likely Eclipsing Binaries or Planets

\begin{tabular}{|c|c|c|c|}
\hline EPIC & Other Name & Coord (J2000) & Notes \\
\hline 212009427 & AD1508 & $083129.87+202437.5$ & Sinsuoidal $P$ with dips superimposed; to appear in Gillen et al. (2017) \\
\hline 211916756 & JS183 & $083727.05+185836.0$ & $\begin{array}{l}\text { Also Libralato et al. (2016), Mann et al. (2016b), Obermeier et al. (2016), Pepper } \\
\text { et al. (2017) }\end{array}$ \\
\hline 211969807 & JC126 & $083832.82+194625.7$ & Also Libralato et al. (2016), Mann et al. (2016b), Pope et al. (2016) \\
\hline 212002525 & UGCSJ083942.02+201745.0 & $083942.03+201745.0$ & To appear in Gillen et al. (2017) \\
\hline 211918335 & $\mathrm{KW} 244=\mathrm{TXCnc}$ & $084001.70+185959.4$ & W Uma type (Whelan et al. 1973) \\
\hline 211970147 & JC193 & $084013.45+194643.7$ & Also Mann et al. (2016b) \\
\hline 211901114 & JS441 & $084135.69+184435.0$ & Possible non-member \\
\hline 211822797 & HSHJ385 & $084138.48+173824.0$ & Possible non-member, very shallow \\
\hline 211946007 & HSHJ430 & $084239.43+192451.9$ & To appear in Gillen et al. (2017) \\
\hline 211922849 & JS550 & $084344.73+190358.5$ & Grazing system \\
\hline 211919680 & HSHJ474 & $084403.90+190112.8$ & Transit signal may be from an unassociated projected background object \\
\hline 211972086 & AD3814 & $085049.84+194836.4$ & To appear in Gillen et al. (2017) \\
\hline
\end{tabular}

recovered, presumably because there are fewer complete cycles in the $\mathrm{K} 2$ campaign. The longer period derived from the LCs is 23.293 and 24.421 days, respectively (with the slightly longer period originating in the LC with fewer counts). Following the same approach as with the rest of the LCs, there is more power in the $\sim 23-24$ day peaks, so that period was assigned as $P_{1}$, the likely rotation period for both EPIC numbers, with the other period assigned to $P_{2}$. However, since these are well-separated peaks, these two LCs were both identified as coming from likely binaries.

Given our initial data amalgamations above (Section 2.5.3), we have $K_{s}=12.222$ and $12.618 \mathrm{mag}$, and no $V$. Our estimates of $\left(V-K_{\mathrm{s}}\right)$ (see Section 2.5.5) are very different for our URAT and Gaia approaches. Gaia measures $G=15.601$ and 16.422 for the two stars, and the $\left(V-K_{\mathrm{s}}\right)$ we thus derive are 4.479 and 5.272. Via the URAT approach, we obtain $f$ magnitudes of 15.05 and 15.53; this is at least consistent with the POSS images that suggest the two stars are very close in brightness. This results in $\left(V-K_{\mathrm{s}}\right)$ of 4.017 and 4.158. We looked via Vizier to find additional direct measurements of $V$ for these stars. NOMAD reports $V=16.12$ and 17.160 , respectively, which would result in $\left(V-K_{\mathrm{s}}\right)=3.898$ and $4.542 \mathrm{mag}$.

Having no abundantly clear answer with respect to what the $\left(V-K_{\mathrm{s}}\right.$ ) should be for each of these stars, we sought input from the $P$ versus $\left(V-K_{\mathrm{s}}\right)$ plot; see Figure 16 . If these stars are "typical" Praesepe members, they should not be outliers. Following the same approach as with the rest of the sample, these stars would both appear within the ensemble, even if both use the $\sim 24$ day period. Taking the shorter period and tying it to the redder (fainter) source, it too is within the distribution at that location, though it is just above the denser population of $\mathrm{M}$ stars. The shorter period does not appear to be appropriate for the brighter (bluer) star; it is an outlier at that location at any $\left(V-K_{\mathrm{s}}\right)$. Using the URAT $\left(V-K_{\mathrm{s}}\right)$ estimates results in the most discrepant points; both stars would be outliers in those cases. Using the NOMAD $V$, the points move slightly right (redder) but are still not necessarily "within the pack" of the rest of the stars.

We suspect that the Gaia-based $\left(V-K_{\mathrm{s}}\right)$ estimates are the best we are going to have at this time. We have retained those, 
Table 5

Binaries in the Literature

\begin{tabular}{|c|c|c|c|c|c|}
\hline EPIC & Other Name & $\begin{array}{l}\text { Coord } \\
(\mathrm{J} 2000)\end{array}$ & $\begin{array}{l}\text { Period(s) } \\
\text { (here, day) }\end{array}$ & $\begin{array}{c}\text { Period(s) }{ }^{\mathrm{a}} \\
\text { (literature, day) }\end{array}$ & Notes \\
\hline \multicolumn{6}{|c|}{ Kovács et al. (2014) } \\
\hline $211935509^{\mathrm{c}}$ & $\mathrm{KW} 539=\mathrm{S} 4$ & $083648.96+191526.4$ & 7.475 & $3.72481,5551$ & HAT-269-0001402; our $P_{\text {rot }}$ is twice theirs, and we believe ours to be correct \\
\hline $211977390^{\mathrm{b}}$ & $\mathrm{KW} 55=\mathrm{S} 27$ & $083749.95+195328.8$ & 6.789 & $7.13827,0.838434$ & $\begin{array}{l}\text { HAT-269-0001352; our peak is broad and the waveform has changes over the campaign; both } 6.8 \\
\text { and } 7.1 \text { may be consistent; no peak at } 0.8 \text { days; also fast outlier in } P \text { versus }\left(V-K_{\mathrm{s}}\right) \text { diagram }\end{array}$ \\
\hline $211949097^{\mathrm{c}}$ & $\mathrm{BD}+19 \mathrm{~d} 2061$ & $083924.97+192733.6$ & $3.940,4.261$ & $4.14405,1.41563$ & $\begin{array}{l}\text { HAT-269-0000761; our peak is broad and both of our periods are probably consistent with theirs; no } \\
\text { peak at } 1.4 \text { days }\end{array}$ \\
\hline $211950081^{\mathrm{c}}$ & KW184 & $083928.58+192825.1$ & 10.175 & $10.4866,4.52284$ & HAT-269-0001490; well-matched $P_{\text {rot }}$; no peak at 4.5 days \\
\hline $211918335^{\mathrm{d}}$ & $\mathrm{KW} 244=\mathrm{TXCnc}$ & $084001.70+185959.4$ & $(0.1914)$ & 0.1915 & HAT-269-000058; WUma EB (Whelan et al. 1973); also listed in EBs table \\
\hline $211933215^{\mathrm{c}}$ & S137 & $084041.90+191325.4$ & 6.112 & $3.11867,897$ & HAT-269-0000850; our peak is correct for our data \\
\hline $211972627^{\mathrm{c}}$ & $\mathrm{KW} 368=\mathrm{S} 155$ & $084110.30+194907.0$ & 9.054 & $9.00657,8.50340$ & $\begin{array}{l}\text { HAT-269-0001570; our waveform undergoes many changes over the campaign, but we do not have } \\
\text { two distinct peaks at } 9.0 \text { and } 8.5 \text { days }\end{array}$ \\
\hline $211935518^{\mathrm{c}}$ & $\mathrm{KW} 434=\mathrm{S} 184$ & $084154.36+191526.7$ & 4.184 & $4.13291,0.951565$ & $\begin{array}{l}\text { HAT-269-0001333; well-matched to } P_{\text {rot }} \text {; we do not find a } 0.95 \text { day peak; also fast outlier in } P \\
\text { versus }\left(V-K_{\mathrm{s}}\right) \text { diagram }\end{array}$ \\
\hline 211947631 & $\mathrm{BD}+19 \mathrm{~d} 2087$ & $084305.93+192615.2$ & 4.736 & $4.65095,7.71605$ & HAT-269-0000465; well-matched $P_{\text {rot }} ;$ no peak at 7.7 days \\
\hline $211969494^{\mathrm{c}}$ & S213 & $084320.19+194608.5$ & 6.382 & $6.18544,648$ & HAT-269-0000913; well-matched $P_{\text {rot }}$ \\
\hline 211896596 & JS655 = FVCnc & $084801.74+184037.6$ & 2.976 & $2.92535,1.01926$ & $\begin{array}{l}\text { HAT-318-0000612; well-matched } P_{\text {rot }} \text {; we do not find a } 1.02 \text { day peak; also appears Mermilliod et al. } \\
\text { (1990) with } P=2.98 \text { days; also fast outlier in } P \text { versus }\left(V-K_{\mathrm{s}}\right) \text { diagram }\end{array}$ \\
\hline \multicolumn{6}{|c|}{ Mermilliod et al. (2009) } \\
\hline 211958646 & HD73081 & $083702.02+193617.2$ & $4.413,2.093$ & 45.97 & Longer than we can detect \\
\hline 211971690 & S12 & $083711.48+194813.2$ & 8.242 & 5.86628 & Our waveform undergoes many changes over the campaign, but no peak is apparent at $\sim 6$ days \\
\hline 211929178 & $\mathrm{KW} 34=\mathrm{BD}+19 \mathrm{~d} 2050$ & $083728.19+190944.3$ & $0.840,0.819$ & 7383 & Longer than we can detect \\
\hline 211959522 & S16 & $083727.54+193703.1$ & 8.873 & 144 & Longer than we can detect \\
\hline 211955820 & $B D+20 d 2130$ & $083727.94+193345.1$ & $\begin{array}{l}3.618,6.605 \\
2.572,2.694\end{array}$ & 47.44 & Longer than we can detect \\
\hline 211947686 & S25 & $083746.61+192618.0$ & 5.933 & 458 & Longer than we can detect \\
\hline 211936163 & HD73210 & $083746.76+191601.9$ & 0.082 & 35.90 & We should have seen a $\sim 36$ day period, but none are apparent \\
\hline $211977390^{\mathrm{b}}$ & $\mathrm{KW} 55=\mathrm{S} 27$ & $083749.95+195328.8$ & 6.789 & 1268 & Longer than we can detect \\
\hline 211983461 & $\mathrm{KW} 58=\mathrm{S} 29$ & $083752.09+195913.9$ & 7.933 & 5567 & Longer than we can detect \\
\hline 211942703 & S38 & $083814.26+192155.4$ & $7.225,9.554,6.369$ & 117 & Longer than we can detect \\
\hline 211990866 & $\mathrm{KW} 100=\mathrm{S} 42$ & $083824.29+200621.8$ & $4.255,4.604,3.971$ & 4.92946 & Comparable to measured periods, but it is unclear whether these are $P_{\text {rot }}$ or $P_{\text {orb }}$. \\
\hline \multicolumn{6}{|c|}{ Mermilliod et al. (1999) } \\
\hline 212034371 & JS102 6 & $083556.94+204934.7$ & 1.21051 & $\cdots$ & Listed as a binary; also fast outlier in $P$ versus $\left(V-K_{\mathrm{s}}\right)$ diagram \\
\hline 212001830 & S11 & $083711.66+201704.9$ & & 1149.5 & $P_{\text {orb }}$ very much longer than we can detect. Noisy and not detected as periodic \\
\hline 211927313 & $\mathrm{KW} 47=\mathrm{BD}+19 \mathrm{~d} 2052$ & $083742.36+190801.5$ & $3.077,3.303,3.626$ & 34.619 & $P_{\text {orb }}$ comparable to our max $P$ but this is not detected \\
\hline 211988454 & $\mathrm{KW} 127=\mathrm{S} 51$ & $083850.00+200403.4$ & $5.909,8.514$ & 13.2803 & $\begin{array}{l}\text { The waveform is very complicated, with more than one period and dense power spectra; } 13.3 \text { days is } \\
\text { not recovered, but could be embedded in the signal }\end{array}$ \\
\hline 211898181 & KW547 & $084037.88+184200.4$ & 2.524 & $\ldots$ & Listed as SB1; also fast outlier in $P$ versus $\left(V-K_{\mathrm{s}}\right)$ diagram \\
\hline $211975006^{\mathrm{d}}$ & $\begin{array}{l}\text { KW367 }=\text { BD } \\
\quad+19 \mathrm{~d} 2077\end{array}$ & $084109.60+195118.6$ & 3.0675 & 1659. & Sinusoidal signal at 3.0675 day very strong; also fast outlier in $P$ versus $\left(V-K_{\mathrm{s}}\right)$ diagram \\
\hline 211912407 & KW439 = HD73994 & $084157.81+185442.2$ & $2.0297,2.102$ & 457.8 & Longer than we can detect; noisy \\
\hline 211989010 & $\mathrm{BD}+20 \mathrm{~d} 2198$ & $084407.33+200436.9$ & $3.682,7.03816$ & 40.6649 & Longer than we can detect but no hint of structure on $\sim 40$ days \\
\hline
\end{tabular}


Table 5

(Continued)

\begin{tabular}{|c|c|c|c|c|c|}
\hline EPIC & Other Name & $\begin{array}{l}\text { Coord } \\
(\mathrm{J} 2000)\end{array}$ & $\begin{array}{l}\text { Period(s) } \\
\text { (here, day) }\end{array}$ & $\begin{array}{c}\text { Period(s) }{ }^{\mathrm{a}} \\
\text { (literature, day) }^{\text {(iter }}\end{array}$ & Notes \\
\hline \multicolumn{6}{|c|}{ Barrado et al. (1998) } \\
\hline 211958646 & HD73081 & $083702.02+193617.2$ & $4.4134,2.093$ & $\ldots$ & Listed as $\mathrm{SB}$ \\
\hline 211991571 & $\mathrm{KW} 146=\mathrm{HD} 73429$ & $083905.23+200701.8$ & 0.7856 & $\ldots$ & Listed as SB \\
\hline 211956096 & KW236 & $083959.82+193400.2$ & 11.509 & $\ldots$ & Listed as SB \\
\hline 212003469 & KW297 & $084028.63+201844.8$ & 8.1555 & $\ldots$ & Listed as SB \\
\hline 211909748 & KW401 & $084130.70+185218.7$ & 2.422 & $\ldots$ & Listed as $\mathrm{SB}$; also fast outlier in $P$ versus $\left(V-K_{\mathrm{s}}\right)$ diagram \\
\hline 211956984 & $B D+20 d 2193$ & $084244.41+193447.8$ & $\begin{array}{c}\text { 0.4794, } 0.9488 \\
0.9937\end{array}$ & $\ldots$ & Listed as SB \\
\hline 211947631 & $\mathrm{BD}+19 \mathrm{~d} 2087$ & $084305.93+192615.2$ & 4.7357 & $\ldots$ & Listed as SB \\
\hline
\end{tabular}

Notes.

${ }^{\text {a }}$ The period given from Mermilliod et al. (2009) is $P_{\text {orb }}$. The two periods given from Kovács et al. (2014) are in the order $P_{\text {rot }}, P_{\text {orb }}$.

b The same star appears explicitly in Kovács et al. (2014) and Mermilliod et al. (2009), as well as Mermilliod et al. (1999) and Barrado et al. (1998).

c Also appears in Mermilliod et al. (1999).

${ }^{\mathrm{d}}$ Also appears in Barrado et al. (1998). 
Table 6

Timescales

\begin{tabular}{|c|c|c|c|}
\hline EPIC & R.A., Decl. (J2000) & Other Name & Timescale (days) \\
\hline 211985684 & $082409.33+200124.9$ & 2MASSJ08240933+2001249 & $\sim 30$ \\
\hline 211995609 & $082635.20+201056.6$ & 2MASSJ08263520+2010567 & $\sim 22$ \\
\hline 211935588 & $082820.20+191530.7$ & 2MASSJ08282020+1915307 & 25.6 \\
\hline 212173061 & $083008.81+233336.4$ & 2MASSJ08300882+2333365 & $\sim 24$ \\
\hline 211787610 & $083055.77+170822.6$ & 2MASSJ08305580+1708223 & $\sim 18$ \\
\hline 211852745 & $083147.19+180322.4$ & 2MASSJ08314719+1803225 & $\sim 23$ \\
\hline 211993704 & $083550.36+200904.5$ & JS98 & 29.7 \\
\hline 211975682 & $083627.12+195154.6$ & $\mathrm{JS} 132=\mathrm{JC} 63$ & $\sim 20.1$ \\
\hline 211813885 & $083713.83+173048.7$ & 2MASSJ08371388+1730487 & $\sim 20$ \\
\hline 211897713 & $083729.40+184135.5$ & 2MASSJ08372941+1841355 & 28.00 \\
\hline 211795569 & $083813.64+171515.7$ & 2MASSJ08381365+1715158 & $\sim 24.2$ \\
\hline 211776073 & $083824.86+165836.3$ & AD2396 = 2MASSJ08382489+1658360 & 22.8 \\
\hline 211935711 & $083936.43+191537.8$ & $\mathrm{JS} 302=\mathrm{JC} 166$ & $\sim 18$ \\
\hline 211950716 & $083941.66+192900.4$ & HSHJ283 & $\sim 11$ \\
\hline 211914960 & $084002.22+185656.9$ & JC183 & 22.4 \\
\hline 211968228 & $084013.78+194455.9$ & JC194 & 20.9 \\
\hline 212112321 & $084111.62+221551.7$ & HSHJ350 & $\sim 35$ \\
\hline 211896120 & $084132.35+184010.7$ & JS439 & 30.3 \\
\hline 211812292 & $084135.58+172927.1$ & $\ldots$ & 35.9 \\
\hline 212005503 & $084151.91+202047.8$ & $\mathrm{JS} 459=2$ MASSJ08415192+2020479 & 21.1 \\
\hline 212108286 & $084207.85+221105.1$ & 2MASSJ08420785+2211051 & $\sim 28$ \\
\hline 211886612 & $084312.92+183150.9$ & JS525 & 17.2 \\
\hline 211983811 & $084332.62+195933.0$ & HSHJ458 = 2MASSJ08433262+1959330 & $\sim 30$ \\
\hline 211939484 & $084552.80+191900.6$ & 2MASSJ08455280+1919006 & 16.7 \\
\hline
\end{tabular}

and assigned the $\sim 24$ day period to the bluer star (EPIC 211934148/JS519) and the $\sim 3$ day period to the redder star (EPIC 211934221 = UGCSJ084307.42+191419.2).

\section{Appendix B Binaries}

There is limited information in the literature on binaries in Praesepe. Here we collect what information we have. Figure 17 has a color-magnitude diagram and a $P$ versus $\left(V-K_{\mathrm{s}}\right)$ diagram of these binaries. We now discuss EBs and binaries from the literature.

\section{B.1. Eclipsing Binaries}

There are 12 objects that we identified in the process of LC inspection as candidate EBs (or planet candidates) in this data set. When we report a period here for these objects, we believe that the $P$ we report and use in our analysis is a rotation period. All of the EBs are listed in Table 4.

We note explicitly here that one object is a known W Umatype EB according to Whelan et al. (1973): EPIC 211918335/ KW244 = TX Cnc. Kovács et al. (2014) also report this as an EB. For this LC, we do not include our 0.191446 day period in our analysis of rotation periods.

\section{B.2. Literature Binaries}

Mermilliod et al. $(1999,2009)$ includes stars in Praesepe that were monitored for spectral binarity (RV variations) over $\sim 20$ years. Kovács et al. (2014) also report photometric periods they attribute to binary components. Barrado et al. (1998) identified spectral binaries from single-epoch observations. Table 5 includes a comparison of the periods derived here to those derived for the binaries in these papers. Many of the periods given in the literature are $\gg 35$ days, so there is no way that we could have obtained that period from these data. For cases where the reported literature periods are both close (and $\lesssim 35$ days), more often than not, we do not recover both periods. It could be that one of the two stars does not have spots, or organized enough spots, to create a detectable signal.

\section{Appendix C Timescales}

As for the Pleiades, we identified some objects in Praesepe that have a repeated pattern, but that we do not think are necessarily due to spot-modulated rotation periods; see Figure 18 for four examples. We describe these as "timescales"; these objects are listed in Table 6. They do not have a preferential color range; see Figure 19 (left). They do, however, tend to have longer periods; see Figure 19 (right).

\section{Appendix D Likely Pulsators}

There are 18 stars that we suspect are pulsators, that is, their power spectra have a "forest" of short-period peaks (see Section 4 and Rebull et al. 2016a). One of these 18 is one of the stars that is too bright for our final best sample. All of these pulsators are listed in Table 7. Most of them are bright 

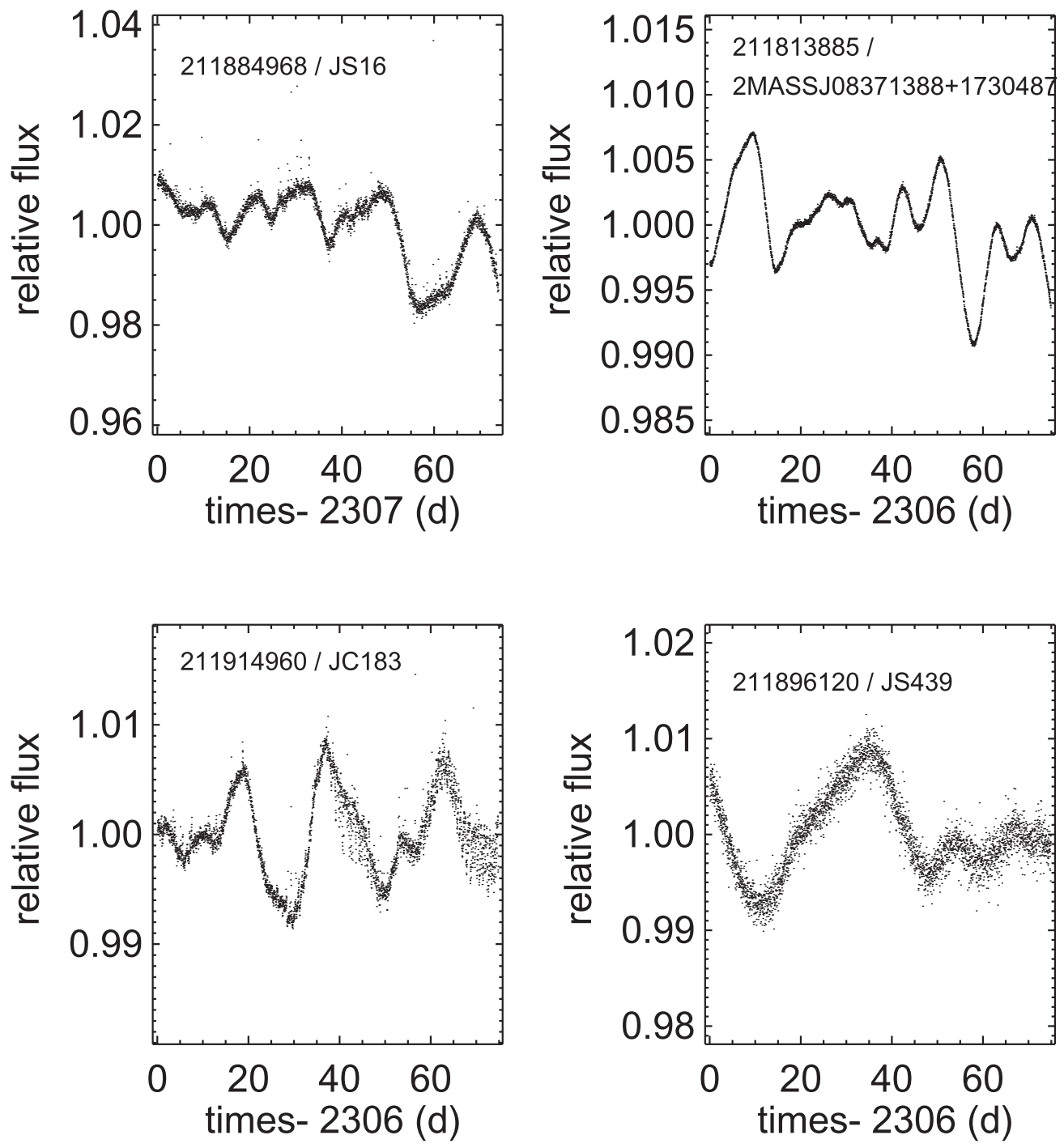

Figure 18. Examples of timescales. Upper left: 211884968/JS16, upper right: 211813885/2MASSJ08371388+1730487, lower left: 211914960/JC183, lower right: $211896120 /$ JS439. These stars have repeated patterns that change considerably with each rotation, so we conclude that they may or may not be rotation periods, and have omitted these periods from the set of rotation periods.

$\left(K_{\mathrm{s}} \lesssim 8.5\right)$, and blue $\left(\left(V-K_{\mathrm{s}}\right) \lesssim 1.1\right)$. There are some stars that are comparably bright and blue, but that do not have a comparable power spectrum; if we derived single periods for them, we retained that period as the rotation period.

\section{Appendix E \\ Non-members}

We identified stars that we took to not be members of Praesepe (see Section 2.5.4, with modifications as per the outliers discussion in Section 3.3 and Appendix G). Table 8 lists these stars. They appear in a color-magnitude diagram and a $P$ versus $\left(V-K_{\mathrm{s}}\right)$ diagram in Figure 20. In general, they are not as well clustered along the main sequence in Praesepe as the members. If they are periodic, these stars generally are outliers in the $P$ versus $\left(V-K_{\mathrm{s}}\right)$ diagram, consistent with being non-members.

\section{Appendix F \\ Bright Giants}

The two stars with $K_{\mathrm{s}}>6$ are EPIC 211984704/39 $\mathrm{Cnc}=\mathrm{HD} 73665$ and EPIC 211976270/HD 73974. These were discarded from our sample as too bright, but we note some characteristics of their LCs here.

The star for which we do not determine a periodic signal using these reduced LCs is EPIC 211976270/HD 73974; it is a giant (K0III; Yang et al. 2015). It is very bright, and the artifacts in the LC reductions we have reflect that. The dominant structure in all the LC versions seems to be the 0.245 day thruster firings.

We find a period in the other star, which is is EPIC $211984704 / 39 \mathrm{Cnc}=\mathrm{HD}$ 73665. It is also a giant (G8III; Yang et al. 2015). We determine only one period, 1.2411 days. Although this object is also very bright, there seems to be a readily apparent $\sim 1$ day oscillation even in the raw LC versions, which is why we retained this period. The phased LC has a lot of scatter in any of the LC versions, admittedly, but there is a peak near 1.24 days that persists across LC versions. But, what does that periodicity represent?

There are four giants in Praesepe, but there are only two K2 LCs. The other two giants are HD73598 (K0III) and HD73710 (G9III). Pasquini et al. (2000) provide $v \sin i$ values for two of them and give an average $v \sin i$ for all four, and they are 

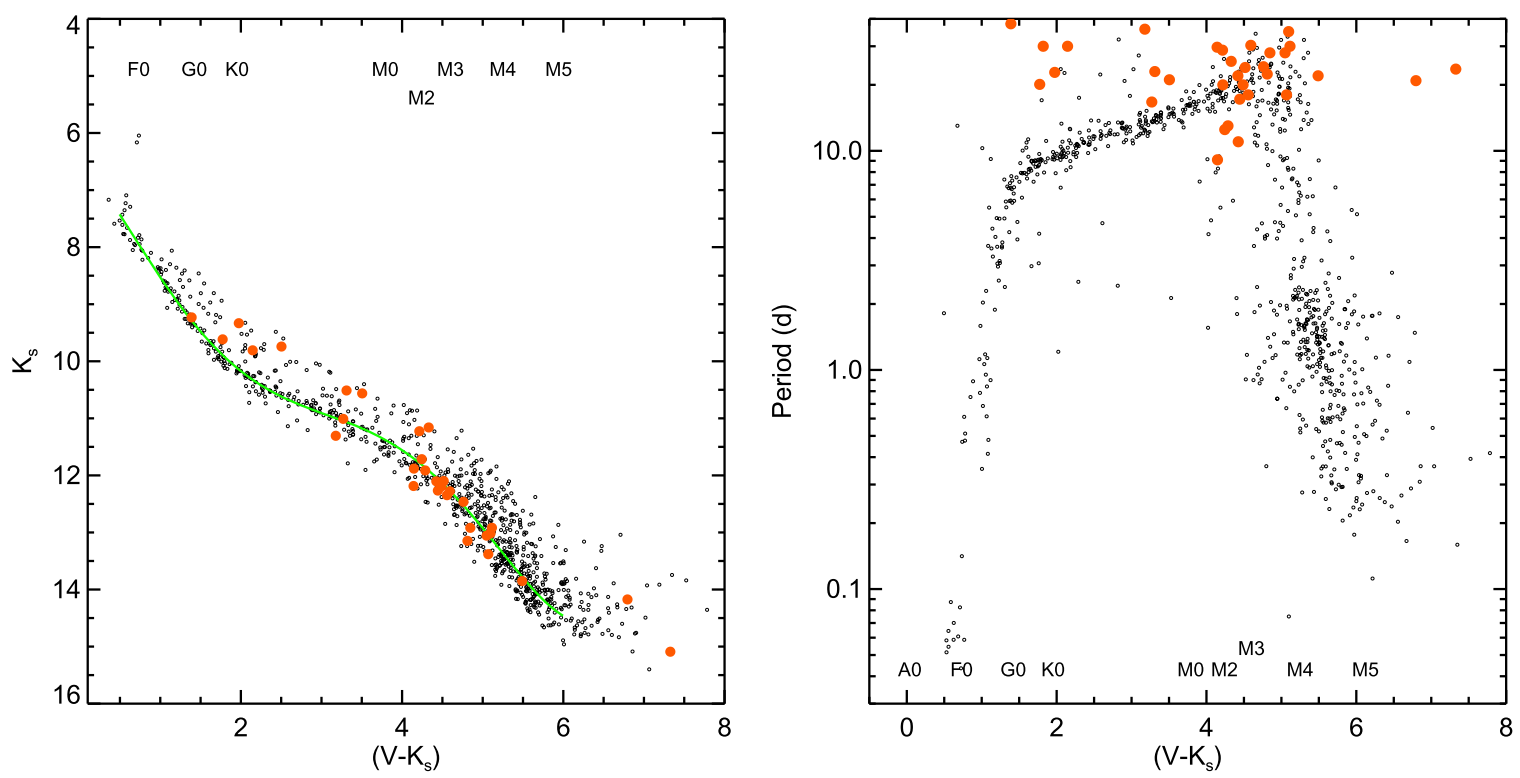

Figure 19. Optical CMD $\left(K_{\mathrm{s}}\right.$ vs. $\left.\left(V-K_{\mathrm{s}}\right)\right)$, left, and $P$ vs. $\left(V-K_{\mathrm{s}}\right)$, right, for the ensemble (small dots), and for stars we identify as having timescales. Non-members are included in these plots because many of the timescale sources are NM. For these timescales, it is not clear if these signatures come from rotationally modulated spots or not. These objects are plotted on the right as if their timescales were periods. Most are long period outliers.

between 1 and $3 \mathrm{~km} \mathrm{~s}^{-1}$, comparable to field red giants. Choi et al. (1995) used long-duration monitoring of calcium HK emission to find rotation periods for three of them. (HD 73598 has $P \sim 159$ day; HD73974 has $P \sim 112$ day; HD73710 has $P \sim 155$ day.) All of those are more or less consistent with the $v \sin i$ values. So, the 1.2 day period we see in the K2 LC cannot be the rotation period.

An estimate of the max power in the oscillations for stars is $\left(\right.$ Brown 1991) $\nu_{\max } / \nu_{\max , \odot} \sim\left(M / M_{\odot} \times\left(T_{\text {eff }} / T_{\text {eff }, \odot}\right)^{3.5} \times\right.$ $\left(L / L_{\odot}\right)^{-1}$. For the Sun, $\nu_{\max , \odot}$ is about $3100 \mu \mathrm{Hz}$ (which converts to about 5 minutes for a period). Assuming that the $T_{\text {eff }}$ for the giants in Praesepe is about $4800 \mathrm{~K}$, and taking their luminosity to be $\sim 60 L_{\odot}$ and their masses as $\sim 2.5 M_{\odot}$, the maximum oscillation power for a Praesepe giant should be at about $4 \mathrm{hr}$. So, the 1.2 day period from the $\mathrm{K} 2 \mathrm{LC}$ is also not likely to be pulsation.

The star is saturated in the $\mathrm{K} 2$ thumbnail, but in general that should result in no derived period, or a period at 0.245 days (or a multiple of that), reflecting the thruster firings (as for the other giant with a K2 LC), not a 1.2 day period. We are not sure if this 1.2 day period is real, nor how to interpret it.

\section{Appendix G \\ Outliers in the $P$ versus $\left(V-K_{s}\right)$ Plot: Detailed Notes}

Section 3.3 above highlights some of the outliers in the $P$ versus $\left(V-K_{\mathrm{s}}\right)$ plot, focusing on both the objects rotating too quickly for their color and those that are going too slowly. Here we focus on individual objects that were covered more broadly above. Figure 21 includes an optical CMD and the $P$ versus $\left(V-K_{\mathrm{s}}\right)$ plot with these objects highlighted; they are listed in Tables 9 and 10.

For the fast-rotating stars, we checked the veracity of their colors and periods, and all of them seemed consistent with the observations. Among the fast-rotating K stars, many of them are known short-period binaries, and we infer that the rest of them are probably binaries as well. Three of the ones with published orbital periods of less than 4 days are $211896596 / \mathrm{JS} 655=\mathrm{FV}$ Cnc, 211975006/KW 367, and 211935518/KW $434=\mathrm{S} 184$ (Mermilliod et al. 1990; Mermilliod \& Mayor 1999). One more (211909748/KW401) is a known SB with a large RV amplitude (Barrado et al. 1998), and another is simply listed as an SB in Mermilliod \& Mayor (1999). Note that there is little literature binary monitoring work done for $\left(V-K_{\mathrm{s}}\right)>3$.

For the slowly rotating stars, we also checked colors and periods. The assessment of their periods is more difficult than other shorter-period stars, as described in Section 3.3 above. Stars particularly subject to this are noted as such in Table 10.

Most of the slow outliers with $1.7<\left(V-K_{\mathrm{s}}\right)<3.4$ and $P>15$ are relatively anonymous stars, with only a few references in SIMBAD. Particularly for the few slowly rotating stars that are photometric binaries in Figure 21, tidal synchronization in a short-period binary system may be a good explanation for the slow rotation. In most cases, they are listed as candidate members in only one or two Praesepe proper motion membership studies. Only one of them has a published RV; that star (212034762/JS409) appears to be a member. We have obtained Keck HIRES spectra for 11 of these stars, which are noted in Table 10 (see Appendix I for details). About half of the ones for which we have spectroscopy appear to have RVs inconsistent with membership. Many of these slow outliers have CMD locations and proper motions generally consistent with being Praesepe members. Some have proper motions consistent with membership, but are slightly low in the CMD; these are noted in Table 10. The lack of detailed information for these stars makes it more likely that they might be non-members, but more information is needed.

\section{Appendix $\mathbf{H}$ Halo Outliers}

The tidal radius of Praesepe is 12.1 pc (Holland et al. 2000), which at a distance of $184 \mathrm{pc}$ is $\sim 3.8$ across. Six of the stars in the original sample of targets with $\mathrm{K} 2 \mathrm{LCs}$ are more than $5^{\circ}$ 
Table 7

Likely Pulsators

\begin{tabular}{|c|c|c|c|c|c|}
\hline EPIC & Other Name & Coord. (J2000) & $\left(V-K_{\mathrm{s}}\right)(\mathrm{mag})$ & Periods (days) & Notes \\
\hline 211953002 & $\mathrm{BR} \mathrm{Cnc}=\mathrm{HD} 73175$ & $083740.70+193106.3$ & 0.588 & $0.0872,0.1219,0.0965$ & F0; $\delta$ Scuti (e.g., Breger 1970) \\
\hline 211983602 & $\mathrm{CY} \mathrm{Cnc}=\mathrm{HD} 73345$ & $083837.86+195923.1$ & 0.530 & $0.051,0.058,0.053,0.067$ & FOV; $\delta$ Scuti (e.g., Hauck 1971) \\
\hline 211951863 & HD 73397 & $083846.95+193003.3$ & 0.779 & $0.5125,0.5950,0.6150,0.7568$ & $\mathrm{~F} 4$ \\
\hline 211957791 & $\mathrm{BS} \mathrm{Cnc}=\mathrm{HD} 73450$ & $083909.09+193532.7$ & 0.625 & $0.0587,0.0644$ & A9; $\delta$ Scuti (e.g., Breger 1970; Breger et al. 2012) \\
\hline 211994121 & HD 73616 & $083958.37+200929.6$ & 0.884 & $0.8876,0.2713,0.5718,0.6137$ & $\mathrm{~F} 2$ \\
\hline 211984704 & $39 \mathrm{Cnc}=\mathrm{HD} 73665$ & $084006.41+200028.0$ & 2.219 & 1.2411 & G8III (dropped as too bright) \\
\hline 211941583 & HD 73712 & $084020.13+192056.4$ & 0.735 & $0.1413,0.4815,0.1179,0.1395$ & Spectroscopic binary; A9V \\
\hline 211931309 & $\mathrm{BV} \mathrm{Cnc}=\mathrm{HD} 73746$ & $084032.96+191139.5$ & 0.686 & $0.0608,0.0637,0.0494,0.0624$ & F0; $\delta$ Scuti (e.g., Hauck 1971) \\
\hline 211973314 & HD 73854 & $084110.67+194946.5$ & 0.850 & $0.7522,0.6970,0.7937,0.7409$ & F5 \\
\hline 211979345 & HD 73872 & $084113.76+195519.1$ & 0.556 & $0.0644,0.0437,0.0771,0.0649$ & A5; $\delta$ Scuti (e.g., Breger et al. 2012) \\
\hline 211935741 & $\mathrm{HI} \mathrm{Cnc}=\mathrm{HD} 73890$ & $084118.40+191539.4$ & 0.627 & $0.070,0.078,0.089,0.059$ & A7V; $\delta$ Scuti (e.g., Hauck 1971) \\
\hline 211945791 & $\mathrm{BX} \mathrm{Cnc}=\mathrm{HD} 74028$ & $084206.49+192440.4$ & 0.529 & $0.058,0.126,0.136,0.055$ & A7V; $\delta$ Scuti (e.g., Breger 1970) \\
\hline 211914004 & $\mathrm{BY} \mathrm{Cnc}=\mathrm{HD} 74050$ & $084210.80+185603.7$ & 0.557 & $0.055,0.053,0.047,0.052$ & A7V; $\delta$ Scuti (e.g., Breger 1970) \\
\hline 211954593 & $\mathrm{BD}+20 \mathrm{~d} 2192$ & $084240.71+193235.4$ & 1.081 & $0.4142,0.6202,1.2397,1.2719$ & F2III \\
\hline 211956984 & $B D+20 d 2193$ & $084244.41+193447.8$ & 1.089 & $0.4794,0.9488,0.9937$ & F6 \\
\hline 212033939 & HD 74135 & $084253.07+204909.1$ & 0.777 & $0.4757,0.3128,0.4135,0.2734$ & A9III \\
\hline 211909987 & HD 74589 & $084520.53+185231.3$ & 0.724 & $0.0434,0.0486,0.0552,0.0535$ & Fo \\
\hline 212008515 & HD 74587 & $084528.25+202343.5$ & 0.767 & $0.0587,0.0513,0.0519,0.0801$ & $\begin{array}{l}\text { A5; } \delta \text { Scuti in SIMBAD and Kovács et al. (2014) but unclear if identified as } \delta \text { Scuti prior to } \\
\text { Kovács et al. (2014) }\end{array}$ \\
\hline
\end{tabular}


Table 8

Non-members

\begin{tabular}{|c|c|c|c|c|c|}
\hline EPIC & R.A., Decl. (J2000) & Other Name & $\left(V-K_{\mathrm{s}}\right)(\mathrm{mag})$ & Period(s) (days) & Notes \\
\hline 212158768 & $083012.13+231336.9$ & 2MASSJ08301213+2313370 & 2.158 & 31.220 & New spectrum has RV inconsistent with membership; also too slow for this color \\
\hline 211897926 & $083150.92+184147.1$ & 2MASSJ08315092+1841470 & 2.282 & 24.521 & New spectrum has RV inconsistent with membership; also too slow for this color \\
\hline 211981509 & $083239.47+195722.2$ & $\mathrm{JC} 10$ & 1.821 & $\ldots$ & $\ldots$ \\
\hline 211803269 & $083300.85+172146.6$ & $\ldots$ & 1.709 & $\ldots$ & $\ldots$ \\
\hline 211933061 & $083316.38+191317.3$ & JS666 & 4.269 & $\ldots$ & $\ldots$ \\
\hline 211885783 & $083334.80+183108.9$ & 2MASSJ08333480+1831089 & 3.279 & $\ldots$ & $\ldots$ \\
\hline 211993704 & $083550.36+200904.5$ & JS98 & 4.419 & $\ldots$ & $\ldots$ \\
\hline 212033649 & $083608.34+204852.8$ & & 4.663 & $\ldots$ & $\ldots$ \\
\hline 212099156 & $083626.89+220016.8$ & 2MASSJ08362690+2200168 & 3.845 & $\ldots$ & $\ldots$ \\
\hline 211955417 & $083649.56+193322.8$ & JC75 & 4.376 & $12.019,5.637,1.235$ & $\ldots$ \\
\hline 211801256 & $083701.08+172005.1$ & 2MASSJ08370108+1720051 & 4.597 & $\ldots$ & $\ldots$ \\
\hline 211930233 & $083703.45+191041.1$ & JC85 & 2.902 & 18.941 & New spectrum has RV inconsistent with membership; also too slow for this color \\
\hline 211833885 & $083707.22+174748.8$ & 2MASSJ08370722+1747487 & 3.676 & $\ldots$ & $\ldots$ \\
\hline 212021664 & $083713.33+203653.6$ & JS170 & 3.957 & 22.048 & Also too slow for this color \\
\hline 212072324 & $083816.83+212953.7$ & JS223 & 4.069 & $\ldots$ & $\ldots$ \\
\hline 211834700 & $083844.47+174829.4$ & ANM690 & 1.436 & $\ldots$ & $\ldots$ \\
\hline 211990313 & $084028.47+200551.2$ & JS363 & 4.598 & $\ldots$ & $\ldots$ \\
\hline 212066519 & $084030.72+212333.1$ & 2MASSJ08403072+2123331 & 4.624 & $\ldots$ & $\ldots$ \\
\hline 212072257 & $084048.52+212949.5$ & 2MASSJ08404852+2129495 & 3.365 & $\ldots$ & $\ldots$ \\
\hline 211984180 & $084053.83+195956.0$ & JS392 & 4.662 & $23.994,1.863$ & Also too slow for this color \\
\hline 211988382 & $084058.92+200359.2$ & JS399 & 4.000 & 25.393 & Also too slow for this color \\
\hline 211954582 & $084113.18+193234.9$ & JC243 & 3.823 & 3.191 & Also too fast for this color \\
\hline 212005583 & $084113.73+202051.7$ & JS417 & 3.301 & 21.610 & Also too slow for this color \\
\hline 212094510 & $084120.89+215454.0$ & 2MASSJ08412090+2154540 & 3.821 & 30.708 & Also too slow for this color \\
\hline 212112522 & $084128.93+221605.3$ & $\ldots$ & 1.355 & $16.018,5.011$ & Also too slow for this color \\
\hline 211812292 & $084135.58+172927.1$ & $\ldots$ & 4.218 & $\ldots$ & $\ldots$ \\
\hline 212084898 & $084136.38+214353.8$ & JS732 & 1.580 & $\ldots$ & $\ldots$ \\
\hline 211784450 & $084155.14+170540.8$ & ANM1065 & 2.676 & $10.992,13.714$ & $\ldots$ \\
\hline 211995547 & $084221.63+201053.1$ & $\ldots$ & 0.994 & 0.591 & $\ldots$ \\
\hline 212137243 & $084221.95+224552.2$ & 2MASSJ08422195+2245521 & 3.657 & 8.950 & Also too fast for this color \\
\hline 211931928 & $084303.65+191215.9$ & JS739 & 5.473 & $\ldots$ & $\ldots$ \\
\hline 212112578 & $084338.80+221609.1$ & KW529 & 2.007 & 8.878 & $\ldots$ \\
\hline 212029850 & $084430.66+204505.0$ & SDSSJ084430.65+204504.7 & 5.162 & $0.213,0.227$ & Also too fast for this color \\
\hline 212011328 & $084501.06+202631.9$ & JS585 & 1.761 & $\ldots$ & $\ldots$ \\
\hline 212069325 & $084811.53+212635.0$ & $\ldots$ & $\cdots$ & $\cdots$ & $\ldots$ \\
\hline 212120476 & $084850.34+222531.9$ & HSHJ506 & 3.171 & 17.097 & New spectrum has RV inconsistent with membership; also too slow for this color \\
\hline 211921444 & $084852.61+190247.0$ & 2MASSJ08485261+1902470 & 4.219 & $\ldots$ & $\ldots$ \\
\hline 211905939 & $085001.55+184852.6$ & 2MASSJ08500156+1848526 & 4.955 & $\ldots$ & $\ldots$ \\
\hline 211939409 & $085125.81+191856.3$ & & $\ldots$ & $\ldots$ & $\ldots$ \\
\hline 211935447 & $085528.96+191523.3$ & 2MASSJ08552896+1915234 & 4.657 & $\ldots$ & $\ldots$ \\
\hline 211875602 & $090222.36+182223.8$ & 2MASSJ09022236+1822238 & 2.371 & 15.490 & New spectrum has RV inconsistent with membership; also too slow for this color \\
\hline
\end{tabular}



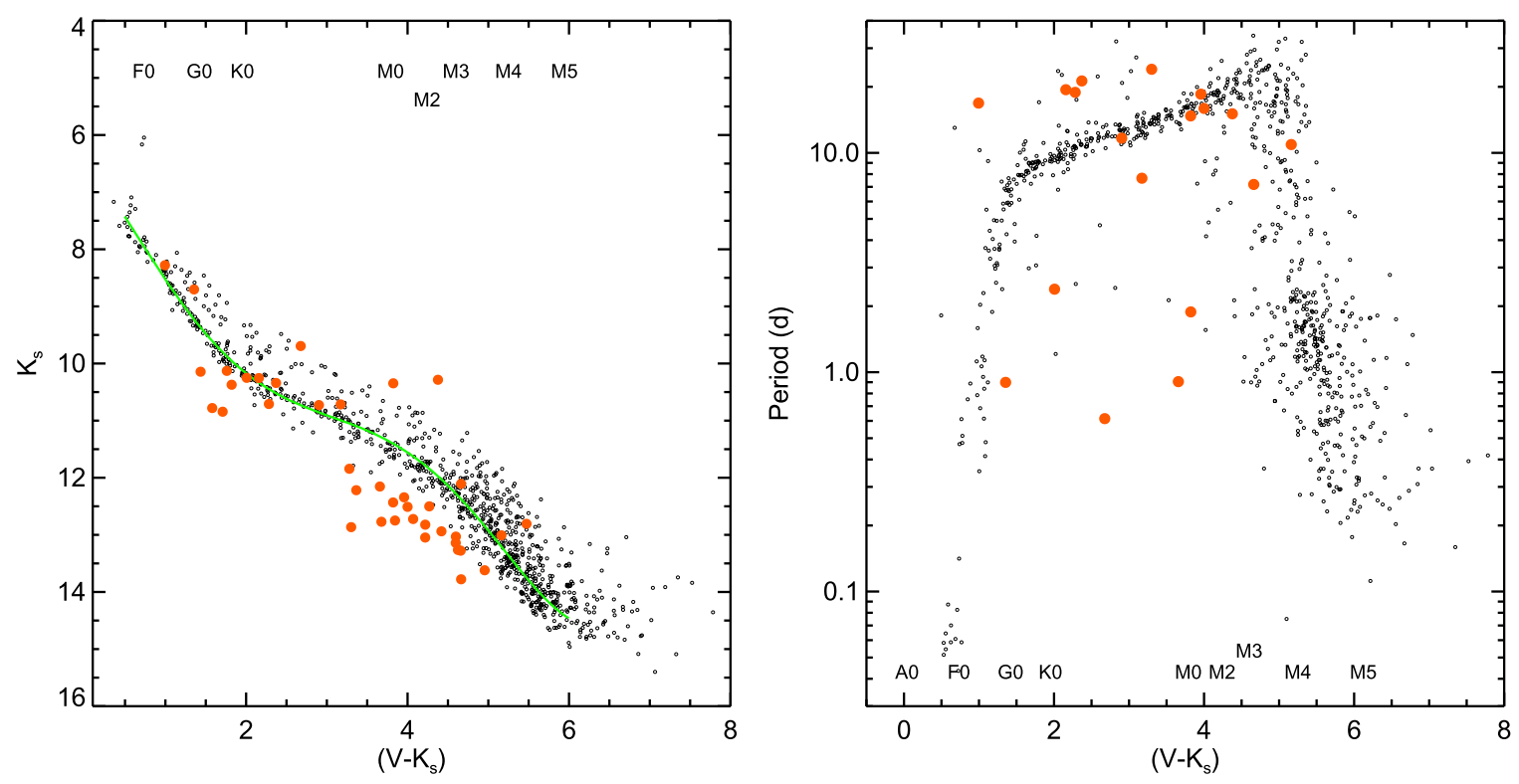

Figure 20. Optical CMD $\left(K_{\mathrm{s}}\right.$ vs. $\left.\left(V-K_{\mathrm{s}}\right)\right)$, left, and $P$ vs. $\left(V-K_{\mathrm{s}}\right)$, right, for the ensemble (small dots), and for stars we identify as non-members. Some do not have identifiable periods, and thus cannot appear on the right. Most are outliers in both diagrams.
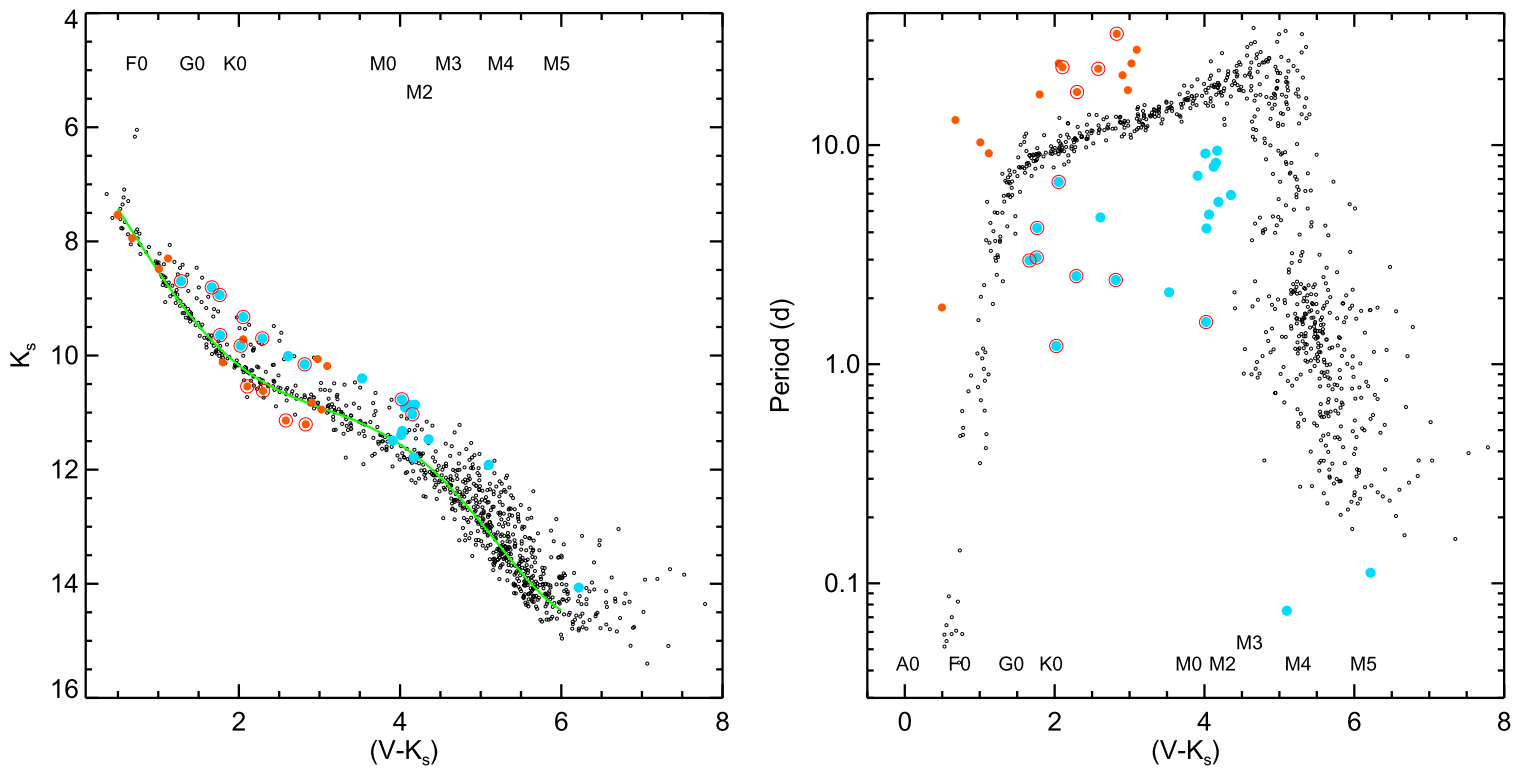

Figure 21. Optical CMD $\left(K_{\mathrm{s}}\right.$ vs. $\left.\left(V-K_{\mathrm{s}}\right)\right)$, left, and $P$ vs. $\left(V-K_{\mathrm{s}}\right)$, right, for the ensemble (small dots), and for stars we identify as rapidly rotating outliers (blue dots) and slowly rotating outliers (orange dots). Non-members have already been removed from these diagrams. An additional red circle around an orange point means that there is some doubt that the star is a member; an additional red circle around a blue point means that the star is a known or suspected short-period spectroscopic binary, where tidal synchronization may have affected the star's rotation period.

away from the cluster center; see Table 11 . They appear in a CMD and the $P$ versus color diagram in Figure 22. One is dropped as a non-member (Section 2.5.4 and Appendix E). Two more are listed as outliers in the $P$ versus $\left(V-K_{\mathrm{s}}\right)$ diagram (Appendix G). In general, these stars are poorly studied, but we have retained those stars that met the rest of our criteria.

\section{Appendix I \\ New Keck Spectra}

We obtained high resolution spectra for several of the anomalously slowly rotating stars (Appendix G) and all of the objects with odd LCs (Section 4.3) using the Keck HIRES spectrograph (Vogt et al. 1994); see Table 12. The observations were taken on one of 2016 October 14, December 22, December 26, or 2017 January 13, UT, and cover the wavelength range roughly $4800-9200 \AA$ at a spectral resolution of $R \approx 45,000$; the spectra were were reduced using the makee software written by Tom Barlow. We measured RVs using the cross correlation techniques in the $r v$ package in $I R A F$, with absolute reference to between three and five (depending on the night) late-type RV standards. Because these are slowly rotating stars, the errors are all under $0.5 \mathrm{~km} \mathrm{~s}^{-1}$ and are determined from the empirical scatter among orders and reference stars for each observation. 
Table 9

Fast Outliers in the $P$ vs. $\left(V-K_{\mathrm{s}}\right)$ Plot

\begin{tabular}{|c|c|c|c|c|c|}
\hline EPIC & R.A., Decl. (J2000) & Other Name & $\left(V-K_{\mathrm{s}}\right)(\mathrm{mag})$ & Period(s) (days) & Notes \\
\hline 211898878 & $081903.68+184236.1$ & 2MASSJ08190368+1842361 & 5.100 & 0.075 & Fastest, bluest M star; it is one of the furthest west sources in Figure 1; it may be a CV \\
\hline 212009427 & $083129.87+202437.5$ & AD1508 = 2MASSJ08312987+2024374 & 4.021 & 1.557 & Likely EB (also listed in EBs table) \\
\hline 211971354 & $083140.45+194754.2$ & HSHJ15 & 4.153 & $8.297,9.374,0.798$ & $\Delta P>6$ day, likely binary; bluest end of sparsely populated $P$ range of $\mathrm{M}$ stars \\
\hline 212034371 & $083556.94+204934.7$ & JS102 = 2MASSJ08355696+2049346 & 2.022 & 1.211 & Binary (also listed in binaries table) \\
\hline 211915860 & $083656.25+185747.9$ & $\mathrm{JS} 159=\mathrm{JC} 80$ & 4.061 & 4.816 & Bluest end of sparsely populated $P$ range of M stars \\
\hline 212021253 & $083728.46+203628.5$ & JS181 & 3.909 & 7.238 & No RVs; bluest end of sparsely populated $P$ range of $M$ stars \\
\hline 211977390 & $083749.95+195328.8$ & $\mathrm{KW} 55=\mathrm{S} 27$ & 2.054 & 6.789 & Binary (also listed in binaries table) \\
\hline 211988628 & $083915.79+200414.0$ & KW566 = JS280 & 4.123 & 7.964 & Bluest end of sparsely populated $P$ range of M stars \\
\hline 211983725 & $083941.03+195928.8$ & KW570 = JS309 & 4.027 & 4.165 & Bluest end of sparsely populated $P$ range of M stars \\
\hline 211918335 & $084001.70+185959.4$ & $\mathrm{KW} 244=\mathrm{TXCnc}$ & 1.282 & $(0.1914)$ & Already removed $P$ as WUma binary (also listed in binaries table) \\
\hline 211920022 & $084005.71+190130.6$ & KW256 = 2MASSJ08400571+1901307 & 2.611 & 4.675 & 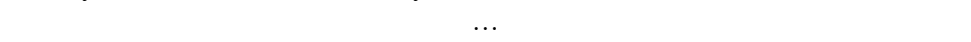 \\
\hline 211984704 & $084006.41+200028.0$ & $39 \mathrm{Cnc}$ & 2.219 & 1.241 & Already removed from sample as too bright (G8III giant) \\
\hline 211915202 & $084029.21+185709.4$ & $\ldots$ & 4.186 & $5.503,2.004$ & Bluest end of sparsely populated $P$ range of M stars \\
\hline 211898181 & $084037.88+184200.4$ & KW547 & 2.290 & 2.524 & Binary (also listed in binaries table) \\
\hline 212013132 & $084044.25+202818.6$ & $\mathrm{JS} 379=2 \mathrm{MASSJ} 08404426+2028187$ & 3.528 & $2.129,4.367,4.053$ & No RVs known \\
\hline 211975006 & $084109.60+195118.6$ & $\mathrm{KW} 367=\mathrm{BD}+19 \mathrm{~d} 2077$ & 1.761 & 3.068 & Binary (also listed in binaries table) \\
\hline 211954582 & $084113.18+193234.9$ & $\mathrm{JC} 243$ & 3.823 & 3.191 & Already NM (also listed in NM table) \\
\hline 211909748 & $084130.70+185218.7$ & KW401 = 2MASSJ08413070+1852188 & 2.816 & 2.422 & Binary (also listed in binaries table) \\
\hline 211935518 & $084154.36+191526.7$ & $\mathrm{KW} 434=\mathrm{S} 184$ & 1.767 & 4.184 & Binary (also listed in binaries table) \\
\hline 212137243 & $084221.95+224552.2$ & 2MASSJ08422195+2245521 & 3.657 & 8.950 & Already NM (also listed in NM table) \\
\hline 211890774 & $084232.06+183528.0$ & JS488 & 4.170 & 9.428 & Marginal outlier; bluest end of sparsely populated $P$ range of $\mathrm{M}$ stars \\
\hline 212066424 & $084300.54+212328.1$ & 2MASSJ08430054+2123281 & 6.217 & 0.112 & Fastest M star in clump; everything about it looks fine \\
\hline 212029850 & $084430.66+204505.0$ & SDSSJ084430.65+204504.7 & 5.162 & $0.213,0.227$ & Already NM (also listed in NM table) \\
\hline 211896596 & $084801.74+184037.6$ & $\mathrm{JS} 655=\mathrm{FVCnc}$ & 1.664 & 2.975 & Binary (also listed in binaries table) \\
\hline 211773459 & $084832.70+165623.6$ & 2MASSJ08483271+1656236 & 4.353 & 5.919 & Bluest end of sparsely populated $P$ range of M stars \\
\hline 211885995 & $084926.76+183119.5$ & 2MASSJ08492676+1831195 & 4.013 & 9.153 & Marginal outlier; bluest end of sparsely populated $P$ range of $\mathrm{M}$ stars \\
\hline
\end{tabular}


Table 11

Outer Halo of Cluster

\begin{tabular}{|c|c|c|c|c|c|}
\hline EPIC & R.A., Decl. (J2000) & Other Name & Period(s) (days) & Distance (deg) & Notes \\
\hline 211999629 & $081259.31+201453.6$ & 2MASSJ08125931+2014537 & & 6.4 & Little additional relevant information found; perhaps below single-star MS \\
\hline 211756862 & $081551.30+164215.9$ & & $1.455,2.627$ & 6.4 & Little additional relevant information found \\
\hline 211984209 & $081615.53+195956.9$ & 2MASSJ08161554+1959570 & 13.754 & 5.6 & Adams et al. (2002) has membership probability of 0.41 ; perhaps below single-star MS \\
\hline 211898878 & $081903.68+184236.1$ & 2MASSJ08190368+1842361 & 0.075 & 5.0 & $\begin{array}{l}\text { One of the fastest rotating } \mathrm{M} \text { stars (listed as fast outlier); Adams et al. (2002) has membership probability } \\
\text { of } 0.39 \text {; a CV rather than member? }\end{array}$ \\
\hline 212148559 & $082333.40+230007.9$ & 2MASSJ08233340+2300079 & & 5.1 & Adams et al. (2002) has membership probability of 0.31; perhaps below single-star MS \\
\hline 211875602 & $090222.36+182223.8$ & 2MASSJ09022236+1822238 & 15.490 & 5.4 & Already dropped as a NM and long-P outlier (listed in corresponding tables); above single-star MS \\
\hline
\end{tabular}



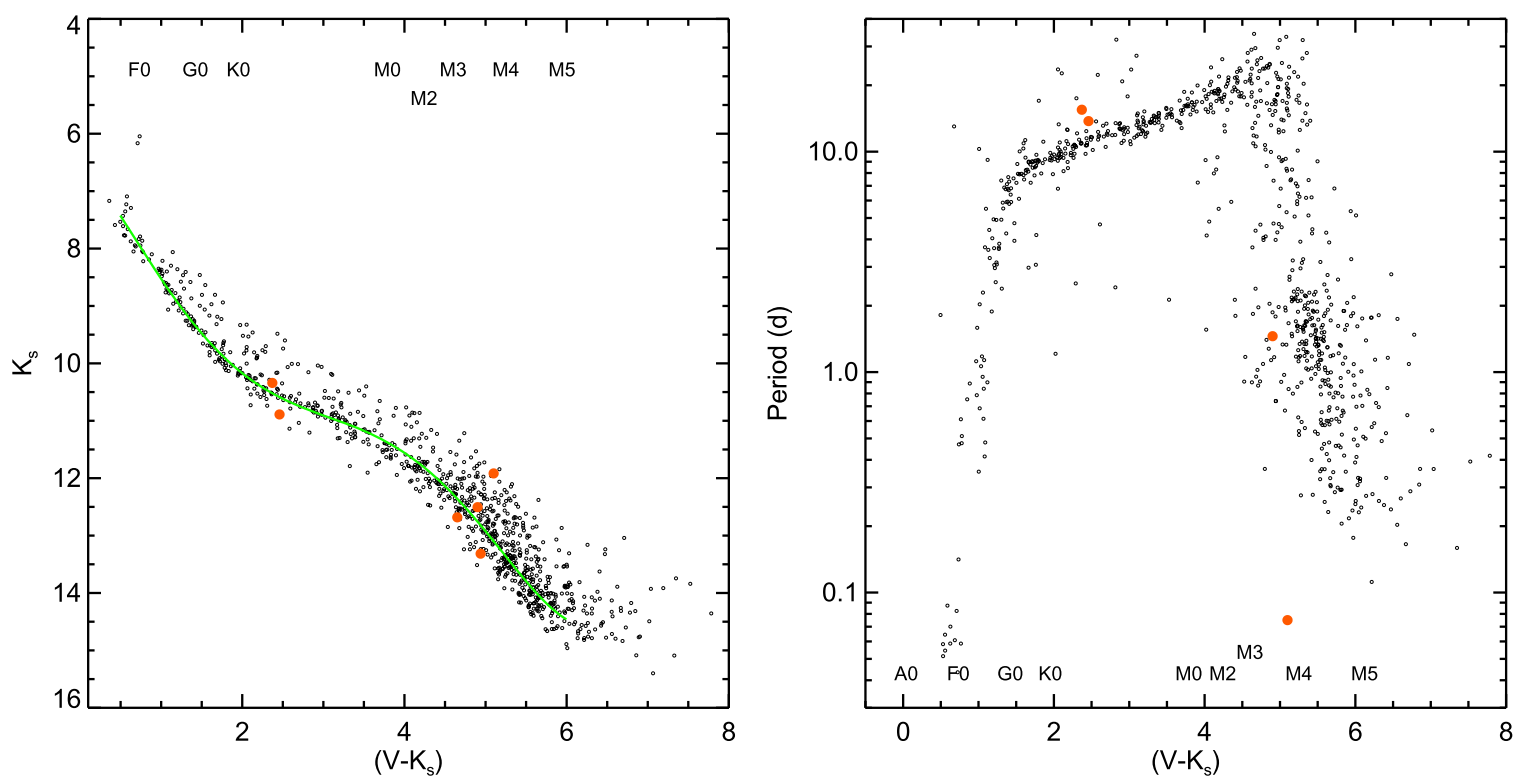

Figure 22. Optical CMD $\left(K_{\mathrm{s}}\right.$ vs. $\left.\left(V-K_{\mathrm{s}}\right)\right)$, left, and $P$ vs. $\left(V-K_{\mathrm{s}}\right)$, right, for the ensemble (small dots), and for stars more than $5^{\circ}$ from the cluster center.

Table 12

Objects with New Keck Spectra

\begin{tabular}{lcccl}
\hline \hline EPIC & R.A., Decl. (J2000) & Other Name & RV $\left(\mathrm{km} \mathrm{s}^{-1}\right)$ & Notes \\
\hline 212158768 & $083012.13+231336.9$ & 2MASSJ08301213+2313370 & $26.36 \pm 0.32$ & Non-member long-P outlier \\
211897926 & $083150.92+184147.1$ & 2MASSJ08315092 +1841470 & $9.08 \pm 0.32$ & Non-member long-P outlier \\
211898294 & $083249.71+184206.2$ & JS14 = JC12 & $27.12 \pm 0.26$ and $41.33 \pm 0.39$ & Double-lined, member long-P outlier \\
212011416 & $083308.44+202637.3$ & 2MASSJ08330845+2026372 & $35.08 \pm 0.31$ & Unusual LC shape, member \\
211930233 & $083703.45+191041.1$ & JC85 & $23.88 \pm 0.26$ & Non-member long-P outlier \\
211915940 & $083800.61+185752.9$ & JS208 & $35.99 \pm 0.47$ & Unusual LC shape, member \\
212034762 & $084111.05+204957.8$ & JS409 & $34.32 \pm 0.30$ & Non-member long-P outlier \\
212098754 & $084212.66+215948.8$ & HSHJ407 & $35.22 \pm 0.35$ & Member long-P outlier \\
211931651 & $084322.40+191200.7$ & AD3196 $=$ CP Cnc & $34.48 \pm 0.26$ & Unusual LC shape, member \\
212120476 & $084850.34+222531.9$ & HSHJ506 & $16.61 \pm 0.31$ & Non-member long-P outlier \\
212027750 & $084914.76+204300.9$ & 2MASSJ08491476+2043009 & $35.95 \pm 0.48$ & Member long-P outlier \\
212008710 & $085502.23+202354.0$ & 2MASSJ08550224+2023540 & $37.07 \pm 0.40$ & Member long-P outlier \\
211875602 & $090222.36+182223.8$ & 2MASSJ09022236+1822238 & $28.57 \pm 0.42$ & Non-member long-P outlier \\
\hline
\end{tabular}

\section{References}

Adams, J., Stauffer, J., Skrutskie, M., et al. 2002, AJ, 124, 1570 Agüeros, M., Covey, K., Lemonias, J., et al. 2011, ApJ, 740, 110 Aigrain, S., Hodgkin, S., Irwin, M., Lewis, J., \& Roberts, S. 2015, MNRAS, 447, 2880

Aigrain, S., Parvianinen, H., \& Pope, B. 2016, MNRAS, 459, 2408

Akeson, R., Chen, X., Ciardi, D., et al. 2013, PASP, 125, 989

An, D., Terndrup, D., Pinsonneault, M., et al. 2007, ApJ, 655, 233

Baker, D., Jameson, R., Casewell, S., et al. 2010, MNRAS, 408, 2457

Barrado y Navascues, D., Stauffer, J., \& Randich, S. 1998, ApJ, 506, 347

Blanco-Cuaresma, S., Soubiran, C., Heiter, U., et al. 2015, A\&A, 577, 47

Boesgaard, A., Roper, B., \& Lum, M. 2013, ApJ, 775, 58

Bonatto, Ch., Bica, E., Girardi, L., et al. 2004, A\&A, 415, 571

Boudreault, S., Lodieu, N., Deacon, N., \& Hambly, N. 2012, MNRAS, 426, 3419

Brandt, T., \& Huang, C. 2015, ApJ, 807, 24

Breger, M. 1970, ApJ, 162, 597

Breger, M., Hareter, M., Endl, M., et al. 2012, AN, 333, 131

Brown, T. 1991, ApJ, 368, 599

Brown, T. 2014, ApJ, 789, 101

Bryja, C., Humphreys, R., \& Jones, T. 1994, AJ, 107, 246

Bryja, C., Jones, T., Humphreys, R., et al. 1992, ApJL, 388, 23

Chambers, K., Magnier, E., Metcalf, N., et al. 2016, arXiv1612.05560
Choi, H.-J., Soon, W., Donahue, R., Baliunas, S., \& Henry, G. 1995, PASP, 107,744

Cody, A. M., Hillenbrand, L., David, T., et al. 2017, ApJ, 836, 41

Coker, C., Pinsonneault, M., \& Terndrup, D. 2016, ApJ, 833, 122 Davenport, J. 2016, arXiv:1610.08563

Delorme, P., Collier Cameron, A., Hebb, L., et al. 2011, MNRAS, 413, 2218

Douglas, S., Agüeros, M., Covey, K., et al. 2014, ApJ, 795, 161

Duncan, D., Baliunas, S., Noyes, R., et al. 1984, PASP, 96, 707

Eggen, O. 1992, AJ, 104, 1482

Friel, E., \& Boesgaard, A. 1992, ApJ, 387, 70

Gaia Collaboration, Prusti, T., de Bruijne, J. H. J., et al. 2016, arXiv:1609. 04172

Gallet, F., \& Bouvier, J. 2015, A\&A, 577, A98

Gillen, E., Hillenbrand, L., David, T., et al. 2017, submitted

Harris, G. 1976, ApJS, 30, 451

Hauck, B. 1971, A\&A, 11, 79

Henden, A., Templeton, M., Terrell, D., et al. 2016, yCat, 2336, 0

Holland, K, Jameson, R., Hodgkin, S., Davies, M., \& Pinfield, D. 2000, MNRAS, 319, 956

Howell, S., Sobeck, C., Haas, M., et al. 2014, PASP, 126, 398

Janes, K., \& Phelps, R. 1994, AJ, 108, 1773

Jardine, M., \& van Ballegooijen, A. 2005, MNRAS, 361, 1173

Jones, B. F., \& Cudworth, K. 1983, AJ, 88, 215

Jones, B. F., \& Stauffer, J. 1991, AJ, 102, 1080 
Kado-Fong, E., Williams, P., Mann, A., et al. 2016, ApJ, 833, 281

Khalaj, P., \& Baumgardt, H. 2013, MNRAS, 434, 3236

Kitchatinov, L., \& Olemskoy, S. 2012, MNRAS, 423, 3344

Klein-Wassink, W. 1927, PGro, 41, 1

Kovács, G., Hartman, J., Bakos, G., et al. 2014, MNRAS, 442, 2081

Kraft, R. 1967, ApJ, 150, 551

Kraus, A., \& Hillenbrand, L. 2007, AJ, 134, 2340

Libralato, M., Nardiello, D., Bedin, L. R., et al. 2016, MNRAS, 463, 1780

Luger, R., Agol, E., Kruse, E., et al. 2016, AJ, 152, 100

Mann, A., Gaidos, E., Mace, G., et al. 2016a, ApJ, 818, 46

Mann, A., Gaidos, E., Vanderburg, A., et al. 2016b, AJ, arXiv:1609.00726

McQuillan, A., Aigrain, S., \& Mazeh, T. 2013, MNRAS, 432, 1203

Meibom, S., Mathieu, R., \& Stassen, K. 2006, ApJ, 653, 621

Melis, C., Reid, M., Mioduszewski, A., Stauffer, J., \& Bower, G. 2014, Sci, 345, 1029

Mermilliod, J.-C. 1981, A\&A, 97, 235

Mermilliod, J.-C., \& Mayor, M. 1999, A\&A, 352, 479

Mermilliod, J.-C., Mayor, M., \& Udry, S. 2009, A\&A, 498, 949

Mermilliod, J.-C., Weis, E., Duquennoy, A., \& Mayor, M. 1990, A\&A, 235,114

Newton, E., Irwin, J., Charbonneau, D., et al. 2016, ApJ, 821, 93

Obermeier, C., Henning, T., Schlieder, J., et al. 2016, AJ, 152, 223

Pace, G., Pasquini, L., \& Francois, P. 2008, A\&A, 489, 403

Pasquini, L., de Medeiros, J., \& Girardi, L. 2000, A\&A, 361, 1011

Perryman, M., Brown, A., Lebreton, Y., et al. 1998, A\&A, 331, 81

Pepper, J., Gillen, E., Parviainen, H., et al. 2017, AJ, 153, 177

Pope, B., Parviainen, H., \& Aigrain, S. 2016, MNRAS, 461, 3399

Radick, R., Thompson, D., Lockwood, G., et al. 1987, ApJ, 321, 459

Rebull, L., Stauffer, J., \& Bouvier, J. 2016a, AJ, 152, 113

Rebull, L., Stauffer, J., \& Cody, A. 2016b, AJ, 152, 114

Salaris, M., Weiss, A., \& Percival, S. 2004, A\&A, 414, 163

Scargle, J. D. 1982, ApJ, 263, 835
Scholz, A., Irwin, J., Bouvier, J., et al. 2011, MNRAS, 413, 2595

Schwarzschild, E., \& Hertzsprung, E. 1913, AN, 196, 9

Skrutskie, M., Cutri, R. M., Stiening, R., et al. 2006, AJ, 131, 1163

Skumanich, A. 1972, ApJ, 171, 565

Soderblom, D., Laskar, T., Valenti, J., Stauffer, J., \& Rebull, L. 2009, AJ, 138,1292

Stauffer, J. 1982, PASP, 94, 678

Stauffer, J., Collier Cameron, A., Jardine, M., et al. 2017, AJ, 153, 152

Stauffer, J., Hamilton, D., \& Probst, R. 1994, AJ, 108, 155

Stauffer, J., Hamilton, D., Probst, R., Rieke, G., \& Mateo, M. 1989a, ApJL, 344,21

Stauffer, J., \& Hartmann, L. 1986, PASP, 98, 1233

Stauffer, J., Hartmann, L., \& Jones, B. 1989b, ApJ, 346, 160

Stauffer, J., Hartmann, L., \& Latham, D. 1987, ApJL, 320, L51

Stauffer, J., Rebull, L., Bouvier, J., et al. 2016, AJ, 152, 115

Stauffer, J., Schultz, G., \& Kirkpatrick, J. D. 1998, ApJL, 499, 199

Taylor, B. 2006, AJ, 132, 2453

Townsend, R., \& Owocki, S. 2005, MNRAS, 357, 251

Upgren, A., Weis, E., \& DeLuca, E. 1979, AJ, 84, 1586

vandenBerg, D., \& Bridges, T. 1984, ApJ, 278, 679

van Leeuwen, F. 2007, A\&A, 474, 653

van Leeuwen, F. 2009, A\&A, 497, 209

Vanderburg, A., \& Johnson, J. 2014, PASP, 126, 948

Vogt, S., Allen, S., Bigelow, B., et al. 1994, PROC SPIE, 2198, 362

Wang, P., Chen, W., Lin, C., et al. 2014, ApJ, 784, 57

Weis, E. 1981, PASP, 93, 437

Werner, M., Roellig, T., Low, F., et al. 2004, ApJS, 154, 1

Whelan, J., Worden, P., \& Mochnacki, S. 1973, ApJ, 183, 133

Wright, E., Eisenhardt, P. R. M., Mainzer, A. K., et al. 2010, AJ, 140, 1868

Yang, X., Chen, Y., \& Zhao, G. 2015, AJ, 150, 158

Zacharias, N., Finch, C., Subasavage, J., et al. 2015, AJ, 150, 101 